University of Louisville

ThinkIR: The University of Louisville's Institutional Repository

Electronic Theses and Dissertations

$5-2012$

\title{
Applications of solid state NMR to cardiolipin and elastin.
}

Tatiana Victorovna Krivokhizhina 1981-

University of Louisville

Follow this and additional works at: https://ir.library.louisville.edu/etd

\section{Recommended Citation}

Krivokhizhina, Tatiana Victorovna 1981-, "Applications of solid state NMR to cardiolipin and elastin." (2012). Electronic Theses and Dissertations. Paper 777.

https://doi.org/10.18297/etd/777

This Doctoral Dissertation is brought to you for free and open access by ThinkIR: The University of Louisville's Institutional Repository. It has been accepted for inclusion in Electronic Theses and Dissertations by an authorized administrator of ThinkIR: The University of Louisville's Institutional Repository. This title appears here courtesy of the author, who has retained all other copyrights. For more information, please contact thinkir@louisville.edu. 


\title{
APPLICATIONS OF SOLID STATE NMR TO CARDIOLIPIN AND ELASTIN
}

\section{By}

\section{Tatiana Victorovna Krivokhizhina}

M.S., Saratov State University, 2003

Ph.D., Saratov State University, 2006

\author{
A Dissertation \\ Submitted to the Faculty of the \\ College of Arts and Sciences of the University of Louisville \\ in Partial Fulfillment of the Requirements \\ for the Degree of
}

Doctor of Philosophy

Department of Chemistry

University of Louisville

Louisville, KY

May 2012 


\section{APPLICATIONS OF SOLID STATE NMR TO CARDIOLIPIN AND ELASTIN}

By

Tatiana Victorovna Krivokhizhina

M.S., Saratov State University, 2003

Ph.D., Saratov State University, 2006

A Dissertation Approved on

May 7, 2012

by the following Dissertation Committee:

$\overline{\text { Dissertation Director Dr. Richard J. Wittebort }}$ 
To My Parents

and

Denis 
Knowing what thou knowest not is in a sense omniscience

Piet Hein 


\section{ACKNOWLEDGMENTS}

I would like to thank my research adviser and excellent swimmer, Dr. Richard J. Wittebort, for his immense enthusiasm and patience, constant encouragement, useful discussions and humor that kept me afloat through Graduate School. In any endeavor I decided to take, from the understanding of NMR of forbidden multiple quantum transitions to the participation in a triathlon with me, Dr. Wittebort was always very supportive, and I am deeply grateful for it.

I have a feeling that no matter how hard I try, there is no way I can thank Dr. Pawel M. Kozlowski enough for the opportunity to join University of Louisville and for the fruitful work in the computational laboratory. I am forever indebted to Dr. Kozlowski for it.

I am grateful to my research advisers Dr. Vladimir V. Nechaev and Dr. Kirill V. Berezin for providing me with experience and education that made my acceptance to the University of Louisville possible.

I would like to express sincere gratitude to the committee members Dr. William L. Dean, Dr. Muriel C. Maurer and Dr. M. Cecilia Yappert for their valuable assistance and time.

I would like to acknowledge faculty and staff members at the University of Louisville for their help, assistance and support over the past years. 
I am grateful to Dr. George R. Pack for his encouragement, inspiration and financial support.

I would like to thank the Chemistry Department of the University of Louisville for the Teaching Assistantship.

I wish to acknowledge Dr. Sengodagounder Arumugam for his valuable assistance and the generous availability of spectrometer time at the James Graham Brown Cancer Center NMR facilities.

I would also like to thank the marathon team members: Dr. Richard P. Baldwin, Dr. Neal J. Stolowich, Dr. Mark S. Mashuta, soon-to-be Dr. Marina V. Malovichko, new-born Dr. Prakash Doiphode and time-tested-canalways-count-on Dr. Frank P. Zamborini. Many thanks to all my friends who made my life in Louisville enjoyable.

Above all, I am grateful to the very special people in my life: my parents, Tatiana and Victor, and my love, Denis. 


\title{
ABSTRACT
}

\section{APPLICATIONS OF SOLID STATE NMR TO CARDIOLIPIN AND ELASTIN}

\author{
Tatiana Victorovna Krivokhizhina
}

May 7, 2012

This dissertation has five chapters. Following an introduction, chapter two describes an investigation of the hypothesis that cardiolipin has a proton storage function in mitochondrial membranes. Chapters three and four present a novel NMR study of the elastic mechanism in elastin. Directions for the future research are given in the last chapter.

To investigate the hypothesis that cardiolipin has a proton storage function, we have used ${ }^{31} \mathrm{P},{ }^{1} \mathrm{H}$ and ${ }^{13} \mathrm{C}$ NMR. A single axially symmetric ${ }^{31} \mathrm{P}$ pattern with reversed anisotropy compared to the static pattern is observed from $\mathrm{pH} 4$ to 9 for tetraoleoyl cardiolipin (TOCL). This confirms that (a) the samples studied are in the biologically relevant liquid crystalline phase, (b) the phosphatidic moieties are equivalent, and (c) TOCL molecules reorient axially about the bilayer normal. Well resolved ${ }^{13} \mathrm{C}$ and ${ }^{31} \mathrm{P}$ MAS NMR spectra show no $\mathrm{pH}$ dependent chemical shifts. Based on a large number of ${ }^{1} \mathrm{H},{ }^{13} \mathrm{C}$ 
and ${ }^{31} \mathrm{P}$ NMR experiments, we conclude that there is no evidence that lamellar multibilayers of TOCL possess a titratable group with a $\mathrm{pK}_{\mathrm{a}}$ in the range of 4 to 9 . We also discuss why the proton storage hypothesis is not energetically realistic.

The mechanism of elastin's recoil is addressed in chapters three and four. The conventional view based on conformational entropy is not supported by preliminary ${ }^{13} \mathrm{C}$ NMR studies, which show no observable increase in backbone ordering with stretch.

To investigate the alternative hypothesis that the hydrophobic effect drives elastic recoil, novel ${ }^{2} \mathrm{H}$ double quantum NMR experiments were implemented. NMR pulse sequences, phase cycles, and methods of analysis were devised to determine the amount of ordered water in elastin and how it changes with stretch, temperature and hydration. Based on the data, which shows a significant increase in the amount of ordered water in all three cases, we conclude that the hydrophobic effect has a significant role in the mechanism of elastin's reversible recoil. Consequently, elastic recoil exploits the increase of the solvent ordering on the hydrophobic surface of the protein when it is stretched, and recoil is driven by a favorable increase of the solvent entropy. 
TABLE OF CONTENTS

PAGE

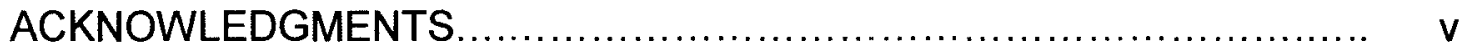

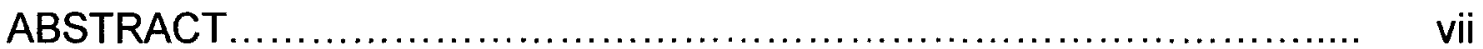

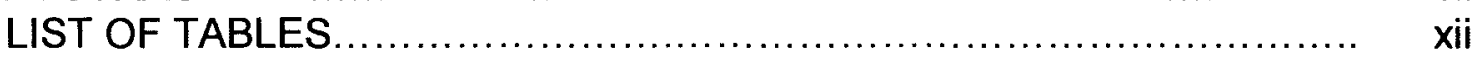

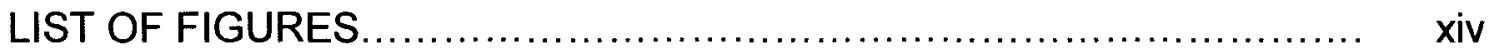

CHAPTER

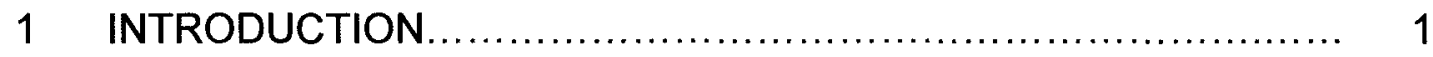

1.1. Cardiolipin ....................................................... 2

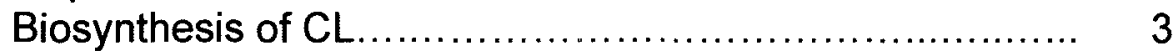

Polymorphism........................................... 4

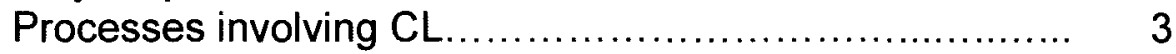

1.2. Elastin........................................................... 5

Elastogenesis........................................... 6

Secondary and tertiary structure .......................... 7

Elasticity mechanisms.................................. 8

1.3. Goals and methods.............................................. 9

Cardiolipin .................................................... 9

Elastin...................................................... 10

2 SSNMR INVESTIGATION OF STRUCTURAL HYPOTHESIS

FOR CARDIOLIPIN ................................................ 11

2.1. Introduction ...................................................... 11

NMR titration.................................................. 13

2.2. Materials and methods.......................................... 14

2.3. Results and discussion....................................... 16

Phase state assignment by ${ }^{31} \mathrm{P}$ SSNMR:

lamellar phase............................................. 16

Implications of static and MAS ${ }^{31} \mathrm{P}$ spectra..................... 19

${ }^{31} \mathrm{P}$ CSA and mobility of the headgroup...................... 21

${ }^{1} \mathrm{H}$ Search for a protonated phosphate......................... 21

Assignment of ${ }^{13} \mathrm{C}$ and ${ }^{1} \mathrm{H}$ NMR spectra........................... 25

NMR titration: ${ }^{13} \mathrm{C}$ and ${ }^{31} \mathrm{P}$ chemical shifts vs. $\mathrm{pH}$............ 30 
2.4. Discussion and conclusions.................................... 33

$3 \quad{ }^{2} \mathrm{H}$ MULTIPLE QUANTUM NMR .................................. 35

3.1. Introduction..................................................... 35

3.2. MQ spectroscopy background ................................ 37

Density operator.......................................... 37

Single transition operators and coherences................. 40

Density operator manipulations:

RF pulses and quadrupolar coupling....................... 43

MQ coherence preparation, evolution and detection.......... $\quad 45$

3.3. Pulse sequence and phase cycle development................... 48

Pulse sequence......................................... 48

$2 \mathrm{Q}$ preparation ........................................... 49

Constant time evolution.................................... 49

Conversion...................................................... 51

Alignment and purge .................................... 51

Phase cycle.............................................. 55

3.4. Types of experiments........................................ 57

Preparation curve................................................. 57

Evolution planes.......................................... 58

3.5. Analysis of experimental data - preparation curve fitting......... $\quad 59$

3.6. Conclusions ..................................................... 65

4 MECHANISM OF ELASTICITY IN ELASTIN ......................... 66

4.1. Introduction ........................................................ 66

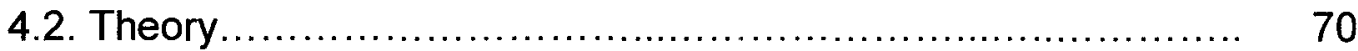

4.3. Materials and methods ......................................... 73

4.4. Results: ${ }^{13} \mathrm{C}$ spectroscopy of the polymer ......................... 83

Residual shielding ......................................... 83

$T_{1}$ Studies.............................................. 85

4.5. Results: MQ NMR studies of the solvent........................ 86

Source of the ${ }^{2} \mathrm{H}$ NMR signals..................................... 86

Effect of stretch and hydration on relaxation times............ 87

Variation of the ordered solvent fraction and

residual coupling with stretch........................................ 92

Effect of temperature on solvent ordering..................... 95

4.6. Discussion and conclusions.................................. 96

5 CONCLUSIONS AND SUGGESTIONS

FOR FUTURE RESEARCH.................................... 101

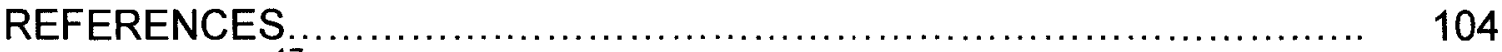

APPENDIX A. ${ }^{17}$ O MULTIPLE QUANTUM NMR........................... 118

A.1. Transition frequencies and operators ........................... 118 
Transition frequencies...................................... 118

Basis operators ........................................ 120

A.2. Scripts...................................................... 122

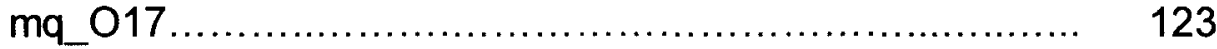

ops6levels.................................................... 125

evol_ThPh................................................ 127

evol_Q_CS................................................ 127

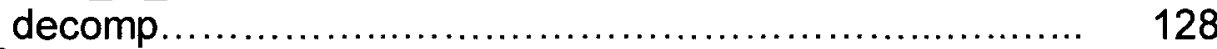



APPENDIX C. ${ }^{2} \mathrm{H}$ SPIN RELAXATION RATES ............................. 131

APPENDIX D. LIST OF SYMBOLS AND ABBREVIATIONS ................. 134

CURRICULUM VITAE................................................ 137 


\section{LIST OF TABLES}

TABLE

PAGE

2.1. Assignments of ${ }^{1} \mathrm{H}$ and ${ }^{13} \mathrm{C}$ NMR spectra of TOCL.................... 29

3.1. Matrix representation of single transition operators, $l_{k}^{i, j}$, for $I=1 \ldots \ldots \ldots 42$

3.2. Phase cycle to cancel $1 Q$ signal..................................... 56

4.1. Chemical shielding and quadrupolar coupling anisotropies

for different nuclei.

68

4.2. $T_{2}^{\text {surf }}$ relaxation times as a function of $P E G$ and

the amount of solvent

78

4.3. Dependence of ${ }^{2} \mathrm{H} 2 \mathrm{Q}$ signal size of elastin five on the amount of solvent in the sample at constant PEG (5 \% PEG) and constant stretch (39\% stretch).

4.4. Tuning and matching conditions for the double resonance probe...... 82

4.5. Longitudinal relaxation time $T_{1}$ for stretched and relaxed elastin fiber

4.6. $T_{2}^{\text {pure }}$ relaxation time of pure solvent as a function of PEG content.....

4.7. $T_{2}^{\text {bulk }}$ relaxation time as a function of stretch and PEG

89

4.8. $T_{2}^{\text {surf }}$ relaxation time as a function of stretch and PEG................ 91 
4.9. ${ }^{2} \mathrm{H} 2 \mathrm{Q}$ signal and parameters from preparation curve expetiment as a function of stretch and PEG................................ 94

A.1. Matrix representations of orthogonalized operators for $I=5 / 2 \ldots \ldots \ldots . \quad 121$ 


\section{LIST OF FIGURES}

FIGURE $\quad$ PAGE

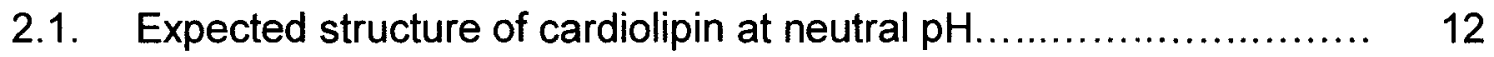

2.2. Static ${ }^{31} \mathrm{P}$ powder patterns of aqueous dispersions of

TOCL and corresponding phase states........................... 18

2.3. Static and MAS ${ }^{31} P$ NMR spectra of TOCL dispersions

in phosphate/citrate phosphate buffer at various $\mathrm{pH} \ldots \ldots \ldots \ldots \ldots \ldots \ldots . . . . . . .20$

2.4. ${ }^{1} \mathrm{H}$ spectra of sodium hydrogen maleate............................... 23

2.5. ${ }^{1} \mathrm{H}$ MAS spectra of TOCL at $\mathrm{pH} 6.5\left(\mathrm{v}_{\text {rot }}=10 \mathrm{kHz}\right)$ and

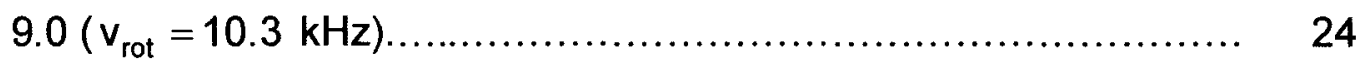

2.6. ${ }^{13} \mathrm{C}$ MAS NMR spectrum of TOCL in phosphate buffer at $\mathrm{pH} 7.0 \ldots \ldots .25$

2.7. Schematic structure of TOCL and nomenclature used for the assignment of ${ }^{13} \mathrm{C}$ and ${ }^{1} \mathrm{H}$ NMR spectra .......................... 26



2.9. ${ }^{13} \mathrm{C}$ MAS NMR spectrum of TOCL in phosphate buffer .............. 28

2.10. ${ }^{1} \mathrm{H}$ MAS NMR spectrum of TOCL in phosphate buffer ................ 28

2.11. ${ }^{13} \mathrm{C}$ MAS NMR spectra of TOCL in phosphate buffer (a) and

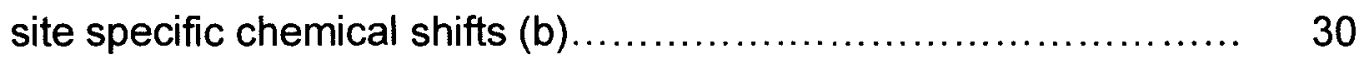

2.12. ${ }^{31} \mathrm{P}$ MAS NMR spectra of TOCL.................................. 31 
2.13. ${ }^{31} \mathrm{P}$ chemical shifts of TOCL $(\bullet)$ and phosphate/citrate phosphate buffer $(x)$ as function of $\mathrm{pH}$

2.14. Analogy between phosphoric acid and phosphodiester



2.16. Model for the estimation of energy of stabilizing bond................. 33

2.17. Model for the axially symmetric reorientation of $\mathrm{CL}$ within the bilayer

3.1. "Pulse sequence" for the preparation of $M Q$ coherence 45

3.2. Energy level scheme for nuclei with $\mathrm{I}=1$. 46

3.3. Simple pulse sequence for MQ NMR 48

3.4. Advanced pulse sequence for the detection of MQ coherence

3.5. Simulated $2 Q$ spectra as a function of the preparation/conversion time, $t_{1}$

3.6. Stack plot of preparation spectra, obtained from elastin sample......

3.7. Example of evolution plane, obtained from elastin sample using 16-steps phase cycle (a) and 64-step phase cycle (b)

3.8. Calculated $P\left(\omega_{q}\right)$ for an axially symmetric residual quadrupolar coupling.

3.9. Illustration of the effect of relaxation and distribution of quadrupolar couplings on preparation curves

3.10. An experimental $2 Q$ preparation curve obtained from native, hydrated elastin fibers 
4.1. The diffusion in a cone model for a water molecule.................. 72

4.2. Parameters for the evaluation of the fraction of ordered water in the sample and the residual quadrupolar coupling from 1Q (a) and $2 Q(b)$ experiments

4.3. Elastin samples at a different stretch............................. 81

4.4. Experimental apparatus (a) Probe with Dewar for temperature controlled experiments. (b) Double resonance ${ }^{1} \mathrm{H} /{ }^{2} \mathrm{H}$ or ${ }^{1} \mathrm{H} /{ }^{13} \mathrm{C}$ static sample NMR probe. (c) Hydrated elastin sample in a $3 \mathrm{~mm}$ NMR tube

4.5. Double resonance circuit............................................ 82

4.6. Setup for the H-D exchange experiment........................... 83

4.7. Static ${ }^{13} \mathrm{C}$ NMR spectra of hydrated elastin.......................... 84

4.8. Ordered water signal after exchange against ${ }^{2} \mathrm{H}_{2} \mathrm{O}(a)$,

${ }^{1} \mathrm{H}_{2} \mathrm{O}$ (b) and ${ }^{2} \mathrm{H}_{2} \mathrm{O}(\mathrm{c})$

4.9. Water ordering as function of temperature......................... 95

4.10. Model for elastin's reversible recoil................................... 100

A.1. Settings for the pulse sequence script, "mq_O17".................... 122

C.1. Dependence of $T_{1}$ (dashed line) and $T_{2}$ (solid line) relaxation times on the correlation time $\mathrm{T}_{\mathrm{c}}$ for intramolecular quadrupolar relaxation (logarithmical axes are used) 
C.2. Linear approximation (solid line) of the dependence of $T_{2}$

(red circles) on the correlation time $\mathrm{T}_{\mathrm{c}}$ in the range

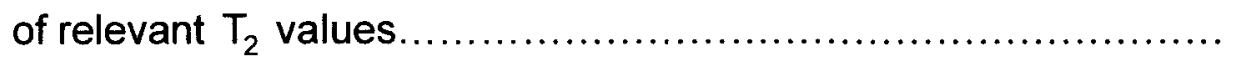

133 


\section{CHAPTER 1}

\section{INTRODUCTION}

Solid-state nuclear magnetic resonance (SSNMR) spectroscopy is a diverse and powerful method for investigation of structure and dynamics of biological molecules and it has been successfully applied for studying globular [17] and fibrous proteins [8-12], as well as membrane proteins [13-18] and lipids [19-24]. SSNMR is particularly beneficial in cases when conventional solution NMR and X-ray crystallography can not be used. These include membrane proteins and phospholipids for which retaining the intact environment is essential for studying structure and function. SSNMR is also an effective method for

investigation of insoluble systems with no long-range order which can not be crystallized.

The present work is dedicated to the development and application of SSNMR methods (1) for investigating a structural hypothesis about the biological role of an important phospholipid of the inner mitochondrial membrane, cardiolipin, and (2) for determining the molecular mechanism of elastic recoil in nature's predominant elastomer, elastin. Our interest in the molecular ordering and dynamics of soft solids (membranes and elastic materials) is the common 
objective. The following is a review of key background information relevant to our studies of cardiolipin and elastin. List of symbols and abbreviations used in the text is given in Appendix D.

\subsection{Cardiolipin}

Cardiolipin $(\mathrm{CL})$ or diphosphatidylglycerol is a phospholipid found primarily in the inner mitochondrial membrane [25] of mammals, invertebrates, yeast and algae. It is also present in plasma membranes of Gram-negative and Grampositive bacteria and this is the basis for the hypothesis about the bacterial origin of mitochondria in eukaryotes [26-27]. CL was first isolated from the beef heart tissue by Mary Pangborn in 1942 [28] and it was named after chemical characterization [29] showed that it is a polyglycero-phospholipid. CL's covalent structure was determined more than 20 years later, in 1964, by LeCocq and Ballou [30].

The unique structural feature that distinguishes $\mathrm{CL}$ from other phospholipids is the presence of two (as opposed to one) phosphatidic acid (PA) moieties per molecule. This is reflected in the name, diphosphatidylglycerol, i.e. $\mathrm{CL}$ is a dimer of PA's bridged by a glycerol. Consequently $\mathrm{CL}$ has four fatty acids and their composition in $\mathrm{CL}$ is highly conserved with mono and diunsaturated 16 and 18 carbon fatty acids being the most abundant [31] in mitochondrial CL. Bacterial $\mathrm{CL}$ is less restricted to fatty acid composition and it favors saturated and monounsaturated 14 to 19 carbon fatty acids [32]. 


\section{Biosynthesis of CL}

In prokaryotes, $\mathrm{CL}$ is synthesized from two molecules of phosphatidylglycerol, in eukaryotes - from one molecule of phosphatidylglycerol and one molecule of cytidinediphosphate-diacylglycerol. As opposed to most phospholipids, which are synthesized in the cytosol adjacent to endoplasmic reticulum, CL is synthesized in mitochondrion. A complete description of the process with all enzymes involved in the synthesis is reviewed in [25]. Interestingly, biosynthesis of $\mathrm{CL}$ is followed by acyl chain remodeling. During a remodeling stage, fatty acid composition is altered to obtain $C L$ enriched with acyl chains specific to the particular organism and tissue. For example, mitochondrial $\mathrm{CL}$ in human tissues are enriched with oleic (18:1n-9) or linoleic $(18: 2 n-6)$ acid. Malfunction of proteins involved in biosynthesis and modification of acyl chain composition of $C L$ are responsible for several diseases [33]. In Barth syndrome, diminution of tetralinoleoyl (18:2n-6) CL and accumulation of monolysocardiolipin cause cardiomyopathy and skeletal weakness. Compositional abnormalities related to increased content of shorter and saturated acyl chains impair mitochondrial function and were reported in brain and stem cell tumors [34].

\section{Polymorphism}

Lipids, in general, are thermotropic and able to reorient their hydrophilic and hydrophobic moieties to take on a wide variety of structures (lipid polymorphism). Organization of CL's molecules in vivo is very sensitive to the characteristics of the local environment such as ionic strength and $\mathrm{pH}$. Although 
most lipids in membranes are in lamellar bilayer phases, some of the lipid molecules exist in non-bilayer phases. Transitions between different phases can be induced by changes in environmental conditions (temperature, $\mathrm{pH}$, presence of ions in the media, etc.) and phase "signatures" can be recognized by SSNMR [35]. CL exists in either inverted hexagonal or lamellar, liquid crystalline phase. The hexagonal phase is favored at low $\mathrm{pH}$ and high ionic strength, while the lamellar phase is stabilized by interaction with other proteins [31]. In addition to environmental factors, the phase of $\mathrm{CL}$ is, to some extent, determined by the fatty acid composition. Longer and more unsaturated acyl chains favor a hexagonal phase [31]. Polymorphism of lipids is an important feature that allows them to perform a variety of biologically important functions. For example, in the contacts between inner and outer mitochondrial membranes, $C L$ present in the hexagonal phase performs transmembrane protein transfer or membrane fusion. However most of the $\mathrm{CL}$ within the membrane exists in the lamellar, liquid crystalline phase.

\section{Processes involving $\mathrm{CL}$}

Despite extensive research, CL's biological function is still not clear. On the other hand, many processes which involve $C L$ are now recognized. The most prominent characteristic of $C L$ is the ability to react with proteins via non-covalent bonding. Proteins of electron transport chain work more efficiently upon CL binding which activates and stabilizes their structure. However, the presence of $\mathrm{CL}$ is not essentially required and these proteins are functional without it. Moreover, CL stabilizes not only separate proteins, but protein complexes of the 
respiratory chain and facilitates electron transfer [36-37]. CL also mediates the apoptosis by activation of caspase-8 [38]. CL increases the mitochondrial membrane potential, thereby making ATP synthesis and protein transfer more efficient.

\subsection{Elastin}

Elastin is an essential fibrous protein that imparts elasticity to tissues and organs that must sustain large amplitude extensions and reversible recoil. It is found in elastic fibers of connective tissue (where it amounts to nearly $90 \%$ of the elastic fiber mass), in vascular tissue and ligaments (50-60\%), in lungs and skin (5-7\%) [39]. Dry elastin is an insoluble, brittle, pale yellow polymer and it acquires elasticity only when swollen in solvent. The first studies of elastin were published nearly a century ago. In 1914, McCartney studied elastin's composition and thermoelastic behavior and these experiments involved separation of elastin from collagen by digestion of collagen with pepsin [40]. Due to its insolubility, the initial studies of elastin employed chemical degradation procedures for the evaluation of amino acid composition. Since the study of soluble material is accessible by a variety of techniques, early research effort was focused on obtaining elastin's soluble counterparts. It was found that elastin is solubilized by oxalic acid ( $\alpha$-elastin) or potassium hydroxide ( $k$-elastin) [41]. Later it was found that, the natural precursor of elastin, tropoelastin, is also soluble. Tropoelastin was initially hard to isolate because once formed and transferred to the extracellular environment, it is converted into insoluble elastin. 
Elastin has been identified in most vertebrates (except primitive jawless fishes) and has not been found in invertebrates [42]. Types of elastin that are actively studied include mammalian (baboon, cat, cow, dog, human, pig, mouse), avian (chicken), teleost (zebrafish) and amphibian organisms (frog) [3334]. Interestingly, there are significant variations in the amino acid sequence of elastins, even in closely related species. The research group of $\mathrm{Dr}$. John Parkinson (Toronto, Canada) created an online data base, ElastoDB http://www.compsysbio.org/elastin/, that summarizes all known elastin sequences (28 sequences). Sequence length varies between species and the average length is about 700 amino acids. It is hypothesized [42-44] that the variations in the sequence allow elastin to adapt to different environments such as encountered by cold-blooded versus warm-blooded animals.

\section{Elastogenesis}

Elastogenesis begins with the secretion of tropoelastin by smooth muscle cells and fibroblasts and transfer onto the cell surface by elastin binding protein $[39,45-49]$. Elastin fiber growth includes coacervation of tropoelastin followed by the deposition of tropoelastin aggregates onto fibrillin microfibrils and their subsequent cross-linking. During coacervation, tropoelastin monomers of about 60-70 kDa self-assemble and form spherical clusters with radius of about 1-3 $\mu \mathrm{m}$ [49]. The fact that optimal conditions for coacervation coincide with physiological conditions $\left(37^{\circ} \mathrm{C}, \quad \mathrm{pH} 7-8,150 \mathrm{mM} \mathrm{NaCl}\right)$ suggests that extracellular coacervation occurs rapidly [39]. The process continues by deposition of these clusters onto fibrillin microfibrils present in the extracellular matrix. Fibrillin 
serves as a scaffold for the growing elastin fiber. Finally, elastin fiber structure is stabilized by intra- and intermolecular cross-linking of tropoelastin. Cross-linking is accomplished by an enzyme that oxidizes lysine sidechains. Unique desmosine and isodesmosine cross-links render elastin fibers insoluble and very durable. In the context of robustness, it is noteworthy that the majority of elastin in mammals is formed during the development and post-natal growth. In mature organisms, the production ceases and only occurs in response to injury [50-51]. The turnover rate of cross-linked elastin is very slow with a half-life of about 70 years [52].

\section{Secondary and tertiary structure}

Tropoelastin has a modular organization with alternating hydrophobic and cross-linking domains of $\sim 30$ and $\sim 20$ residues, respectively. Strictly speaking, the presence of alternating elastomeric and cross-linking domains is a common feature of all elastomers [53]. Tropoelastin's hydrophobic domains are rich in the non-polar amino acids glycine, alanine, valine and proline. Interaction of hydrophobic domains is responsible for self-assembly of tropoelastins [39]. In addition, two highly conserved cysteine residues from the C-terminus of tropoelastin are important for self-assembly. Their removal greatly reduces the self-assembly rate [54]. Proline in hydrophobic domains is important for preserving elastin-like fibrous structure, since its depletion or substitution with

glycine causes formation of amyloid-like structures [55-57]. Cross-linking domains are rich in alanine and lysine. Desmosine cross-links are formed by the lysines in the cross-linking domains. Study of cooperativity between domain 
types shows that they are not independent. In particular, substitutions in crosslinking domains change structure and dynamics in hydrophobic domains [58].

Proper assembly and cross-linking of elastin is essential for health. There are severe diseases such as cutis laxa (inelastic skin), SVAS (supravalvular aortic stenosis, narrowing of the opening of aortic valve) [59], elastoderma (laxity of skin) [60] which are induced by mutations in the gene encoding elastin.

Circular dichroism studies of soluble, chemically modified elastins by Gotte [61] in the 1970's supported a twisted rope organization of elastin fibers with a-helical and $\beta$-turn conformations. Later, in 1980, Aaron and Gosline demonstrated that single water-swollen elastin fibers are optically isotropic suggesting a random coil network. The same result was indicated in isotope labeling structure studies by Perry et al. [62]. Based on the results of ${ }^{13} \mathrm{C}$ and ${ }^{2} \mathrm{H}$ SSNMR studies, Pometun et al. concluded that the backbone in hydrated elastin is highly disordered [63]. Current knowledge about elastin's structure is based on experiments that show conformationally flexible random-coil backbone [6269].

\section{Elasticity mechanisms}

Since physical properties of hydrated elastin are similar to those of rubber, i.e. it is highly flexible and extensible, the similarity in their structural organization and elasticity mechanisms was assumed. From the perspective of the network organization, elastin is similar to rubber, which is composed of highly mobile (kinetically free) cross-linked polyisoprene chains, which are 
similar to hydrophobic domains within cross-linked tropoelastin chains. With stretch, the polymer has fewer conformations. Therefore recoil is driven by increase in entropy of the polymer. That said, the fact that elastin is only elastic when swollen in solvent requires consideration of the role of solvent in elasticity, i.e. a mechanism that is different from rubber elasticity.

Thus, the mechanism of elasticity is still a subject of debate. At present, there are two main theories. The rubber elasticity mechanism based on configurational entropy of the polymer and a mechanism based on the hydrophobic effect, i.e. a decrease in solvent entropy.

\subsection{Goals and methods}

\section{Cardiolipin}

In chapter two, we examine the hypothesis that formation of strong $\mathrm{H}$-bonds within the headgroup of $\mathrm{CL}$ significantly raises headgroup $\mathrm{pK}_{\mathrm{a}}$ 's from the value characteristic of a phosphodiester group. ${ }^{1} \mathrm{H},{ }^{31} \mathrm{P}$ and ${ }^{13} \mathrm{C}$ NMR titrations are used. Protons stabilized in $\mathrm{H}$-bonds have distinct downfield shifts and in same cases can be observed in ${ }^{1} \mathrm{H}$ NMR spectra. Intramolecular $\mathrm{H}$-bonds with headgroup phosphates should also change ${ }^{31} \mathrm{P}$ and ${ }^{13} \mathrm{C}$ chemical shifts. Therefore ${ }^{31} \mathrm{P}$ and ${ }^{13} \mathrm{C}$ spectra at different $\mathrm{pH}$ are useful for the determination of this putative structural change. For these studies, CL samples were prepared in the biologically relevant lamellar, liquid crystalline phase. This was confirmed by ${ }^{31} \mathrm{P}$ NMR. A detailed investigation of putative structural change in CL's headgroup is important for understanding the biological role of $\mathrm{CL}$ at the molecular level. 


\section{Elastin}

To investigate the mechanism of reversible elasticity in elastin, we test the two qualitatively distinct hypotheses for elastin's mechanism of recoil. In both hypotheses, elastic recoil is an entropy driven order to disorder process, however, the molecular sources of entropy are different, either the protein backbone or the solvent. To experimentally determine the relative importance of these two mechanisms in the function of elastin, we measure changes in ordering of the backbone, using ${ }^{13} \mathrm{C} N M R$, and the solvent, using ${ }^{2} \mathrm{H} M Q$ NMR, as a function of stretch, hydration and temperature. These studies are fundamental for the design of biocompatible elastomeric materials for wound repair, construction of artificial blood vessels [51] and other applications of elastin in tissue engineering. 
CHAPTER 2

SSNMR INVESTIGATION OF STRUCTURAL HYPOTHESIS FOR

CARDIOLIPIN

\subsection{Introduction}

The involvement of anionic phospholipids in proton conduction in the membranes of energy producing organelles was hypothesized in the early 1980's [70]. Michel et al. observed lateral relocation of protons along the membranes of halobacteria [71]. Haines suggested a mechanism for this process [70]. According to his hypothesis, the headgroups of anionic lipids in the membrane serve as proton carriers. This is in contrast to the previous notion about proton transfer via bulk aqueous phase. Protons in such membranes are transferred laterally via dynamic formation of acid-anion pairs, in which two anions share a single proton via a hydrogen bond. Later, in the early 1990's, a similar mechanism of proton stabilization was hypothesized for cardiolipin (CL) [72]. An unusual buffering capacity of $\mathrm{CL}$ in $\mathrm{KCl}$ was observed during a potentiometric titration. The titration curve indicated a $\mathrm{pK}_{\mathrm{a}}$ value above 7 [72]. Haines and coworkers suggested the formation of a cyclic structure within the headgroup that stabilizes the proton by hydrogen bonding thereby increasing a higher $\mathrm{pK}_{\mathrm{a}}$. 
Unlike most glycerophospholipids, CL contains two phosphatidic acid moieties, which are esterified to a central, bridge glycerol (Figure 2.1). Based on the $\mathrm{pK}_{\mathrm{a}}$ of simple phosphodiesters, both phosphates in the headgroup are expected to be negatively charged at physiological conditions.

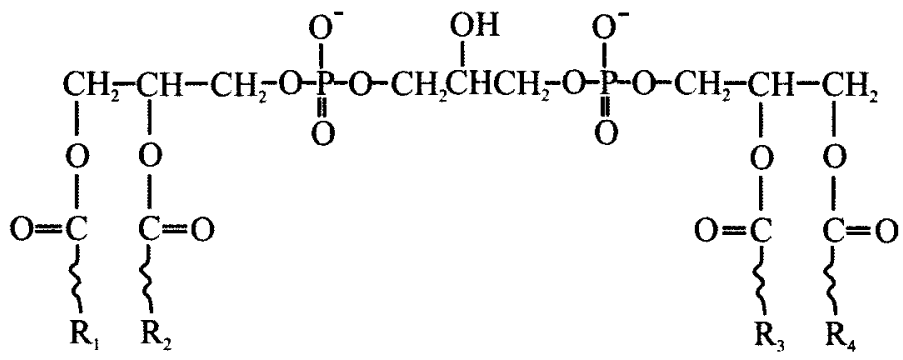

Figure 2.1. Expected structure of cardiolipin at neutral $\mathrm{pH}$.

A suggested explanation of unexpected buffering capacity of $\mathrm{CL}$ above physiological $\mathrm{pH}$ reported in [72] is the formation of an $\mathrm{H}$-bond between a protonated phosphate $\left(\mathrm{pK}_{\mathrm{a} 2}\right)$ and the central glycerol hydroxyl that also donates a hydrogen bond to the unprotonated phosphate $\left(\mathrm{pK}_{\mathrm{a} 1}\right)$. Another important feature of cardiolipin's titration curve, noted in [72], is the drifting of $\mathrm{pK}_{\mathrm{a} 2}$ in the course of titration. That is, deprotonation of the acid-anion pair is not characterized by a single $\mathrm{pK}_{\mathrm{a}}$. The explanation of this effect, given in [72], is destabilization of the headgroup in a bicyclic structure during the titration.

Experimental evidence for the participation of $\mathrm{CL}$ in stabilization of structure and activation of many mitochondrial redox enzymes involved in oxidative phosphorilation [73-75] indicates the importance of $\mathrm{CL}$ for energy transduction. But no molecular mechanism was suggested until Hanes' hypothesis. This structural hypothesis assigns proton storage capacity to CL. 
Our goal is to test the structural hypothesis and identify possible titratable groups by performing a $\mathrm{CL}$ titration monitored by the $\mathrm{pH}$ dependence of ${ }^{13} \mathrm{C}$ and ${ }^{31} \mathrm{P}$ chemical shifts. To address this hypothesis with a physiologically relevant sample, we prepared unsonicated, lamellar $C L$ multibilayers from which we obtained high resolution magic angle spinning (MAS) ${ }^{1} \mathrm{H},{ }^{13} \mathrm{C}$ and ${ }^{31} \mathrm{P}$ NMR spectra.

\section{NMR titration}

To understand the effects of titration on the NMR spectrum, let us consider the following. Conversion of protonated species A (acid) into deprotonated $B$ (conjugate base) results in different chemical shifts $\delta_{A}$ and $\delta_{B}$ for $A$ and $B$, respectively. With the assumption of fast chemical exchange, a single chemical shift is observed, $\delta_{o b s}$, that is a weighted average of the chemical shifts of $A$ and $B$ :

$$
\delta_{o b s}=\delta_{A} f_{A}+\delta_{B} f_{B}=\delta_{A} \frac{A}{A+B}+\delta_{B} \frac{B}{A+B},
$$

where $f_{A}$ and $f_{B}$ are mole fractions of $A$ and $B$ respectively. With the equilibrium expression for the acid dissociation

$$
K_{a}=\frac{H^{+} B}{A},
$$

we obtain

$$
\frac{A}{A+B}=\frac{A}{A+\frac{K_{a} A}{H^{+}}}=\frac{1}{1+\frac{K_{a}}{H^{+}}}=\frac{H^{+}}{H^{+}+K_{a}}
$$

and 


$$
\frac{B}{A+B}=\frac{B}{\frac{H^{+} B}{K_{a}}+B}=\frac{1}{\frac{H^{+}}{K_{a}}+1}=\frac{K_{a}}{H^{+}+K_{a}}
$$

Substituting into the expression for $\delta_{\text {obs }}$, we obtain

$$
\delta_{o b s}=\frac{\delta_{A} H^{+}}{H^{+}+K_{a}}+\frac{\delta_{B} K_{a}}{H^{+}+K_{a}}
$$

which this is rearranged to give,

$$
\mathrm{H}^{+}\left(\delta_{\mathrm{obs}}-\delta_{\mathrm{A}}\right)=\mathrm{K}_{\mathrm{a}}\left(\delta_{\mathrm{B}}-\delta_{\mathrm{obs}}\right)
$$

After taking logarithms of both sides, we obtain

$$
p H=p K_{a}+\log \frac{\left(\delta_{o b s}-\delta_{A}\right)}{\left(\delta_{B}-\delta_{o b s}\right)}
$$

This is the the Henderson-Hasselbalch equation:

$$
\mathrm{pH}=\mathrm{pK}_{\mathrm{a}}+\log \frac{\mathrm{B}}{\mathrm{A}}
$$

in a form that can be used to determine a titration curve obtained by plotting chemical shifts against $\mathrm{pH}$. This provides $\mathrm{pK}_{\mathrm{a}}$.

\subsection{Materials and methods}

The choice of a cardiolipin is dictated by two conditions - the lipid has to be in the lamellar, liquid crystalline phase at room temperature and it has to be common in eukaryotes. It has been found that mono- and diunsaturated chains composed of 16 and 18 carbons occur more often in eukaryotic CL [31]. To choose a $C L$ convenient for studying the liquid crystalline $L_{a}$ phase, we have compared the more complete data on the temperatures of gel to liquid crystalline 
phase transitions of glycerophospholipids to the more limited data for CL. Phosphatidylcholine, phosphatidylglycerol and phosphatidylserine containing myristyl $\left(C_{14}\right.$, saturated) moieties undergo gel to liquid crystalline phase transitions at 23,23 and $35^{\circ} \mathrm{C}$ respectively [76]. These transition temperatures decrease systematically when myristic acid is replaced by oleic acid $\left(C_{18}\right.$, unsaturated) to $-20,-18$ and $-11^{\circ} \mathrm{C}$, respectively [76]. Based on this, and the fact that the tetramyristoyl $\mathrm{CL}$ gel to liquid crystalline phase transition occurs at $40^{\circ} \mathrm{C}$ [77] we chose tetraoleoyl $18: 1 \mathrm{CL}$, since at room temperature this lipid is reasonably expected to be in the lamellar, liquid crystalline phase.

Natural abundance (unlabelled) 1,1',2,2'-tetraoleoyl cardiolipin (TOCL) in lyophilized powder form was purchased from Avanti Polar Lipids Inc. and used without further purification. In this synthetic cardiolipin, all four fatty acids are monounsaturated oleic acid (18:1n-9).

For experiments at $\mathrm{pH}$ values from 2.2 to 5.5 , the buffer was phosphate/citrate prepared from $100 \mathrm{mM}$ citric acid and $200 \mathrm{mM} \mathrm{Na} \mathrm{HPO}_{4}$. For $\mathrm{pH}$ values from 6.0 to $9.0100 \mathrm{mM} \mathrm{Na}$ phosphate buffer was prepared by mixing appropriate volumes of $200 \mathrm{mM} \mathrm{NaH} \mathrm{PO}_{4}$ and $200 \mathrm{mM} \mathrm{Na}_{2} \mathrm{HPO}_{4}$ and adding an equal volume of distilled water. Sample preparation included equilibration of solid cardiolipin in excess buffer followed by separation of the lipid and buffer phases by centrifugation. The $\mathrm{pH}$ of supernatant was measured after repeating of equilibration process at least three times to ensure $\mathrm{pH}$ of cardiolipin. Simple vortexing was used to mix lipid and buffer. Then the lipid phase was transferred to the MAS rotor. Once ${ }^{13} \mathrm{C},{ }^{31} \mathrm{P}$ spectra were obtained, the same sample was 
used for another round of buffer equilibration and spectra accumulation. With this procedure, the same lipid sample was used for obtaining data at different $\mathrm{pH}$ values.

Ultrasonication, which alters the physical state of the membrane [78], is unnecessary to obtain high spectral resolution. No sonication was used in this study. Samples were concentrated by several rounds of centrifugation at $10,000 x g$.

For HSQC solution NMR experiments, used to make resonance assignments, TOCL was dissolved in deuterated chloroform $\left(\mathrm{CDCl}_{3}\right)$.

${ }^{31} \mathrm{P}$ static and MAS spectra were recorded on a homebuilt NMR spectrometer (11.7 Tesla), and ${ }^{13} \mathrm{C}$ and ${ }^{1} \mathrm{H}$ MAS and solution NMR spectra were obtained on a Varian Inova-800 NMR spectrometer (18.8 Tesla). Spectra were recorded several times with different samples and all spectra were found to be reproducible.

\subsection{Results and discussion}

Phase state assignment by ${ }^{31} \mathrm{P}$ SSNMR: lamellar phase

Phase behavior of phospholipids can be evaluated by ${ }^{31} \mathrm{P}$ SSNMR, since various phase states have unique ${ }^{31} \mathrm{P}$ static powder patterns. Possible lipid phases include hexagonal or inverted hexagonal phase $\left(H_{\| 1}\right)$, micellar hexagonal phase $\left(H_{1}\right)$, liquid crystalline phase $\left(L_{\alpha}\right)$ and gel phase $\left(L_{\beta}\right)$. The lamellar, liquid crystalline phase is a "pile" of $L_{a}$ lipid bilayers with a thin layer of water solution between lamellae. The lamellar phase is also denoted as $L_{\alpha}$ and it is the 
biologically relevant phase for membrane studies. Figure 2.2 illustrates ${ }^{31} \mathrm{P}$ static powder patterns of TOCL aqueous dispersions in two phase states. The hexagonal phase has a static powder pattern with positive span while the lamellar phase has an axially symmetric pattern and the span, $s=\sigma_{33}-\sigma_{11}$, has the opposite sign. In sonicated lipid dispersions, lipids are contained in spherical, unilammelar vesicles so chemical shift anisotropy (CSA) is averaged to a relatively narrow line due to isotropic reorientation of the vesicles. The gel phase has larger span than $L_{\alpha}$ and a non-zero asymmetry because of restricted mobility.

The static ${ }^{31} \mathrm{P}$ NMR spectra of TOCL (Figure 2.3) have ${ }^{31} \mathrm{P}$ shielding anisotropy of $33.6 \mathrm{ppm}$ with negative span. This is consistent with the spectrum of $L_{\alpha}$ phase TOCL reported in [79] and shown in Figure 2.2. Therefore, the samples of TOCL studied in sodium phosphate buffer $\mathrm{pH}$ from $\mathrm{pH} 4$ to $\mathrm{pH} 9$ are in the $L_{\alpha}$, lamellar, multibilayers phase. 


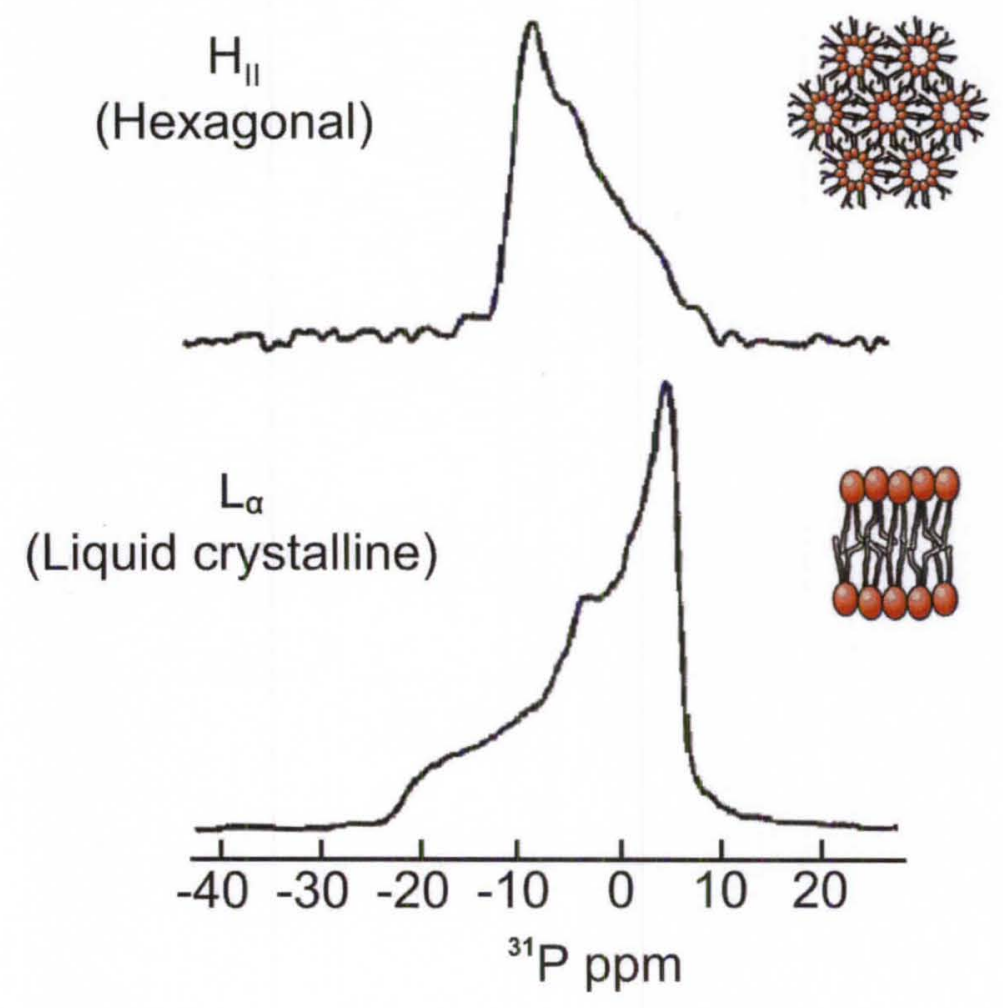

Figure 2.2. Static ${ }^{31} \mathrm{P}$ powder patterns of aqueous dispersions of TOCL and corresponding phase states. 


\section{Implications of static and MAS ${ }^{31} \mathrm{P}$ spectra}

Static and MAS ${ }^{31} \mathrm{P}$ spectra of lamellar CL multibilayers at different $\mathrm{pH}$ are given in Figure 2.3. The sharp peak in the center of the powder pattern corresponds to the buffer phosphate and its chemical shift changes with titration. In effect, it serves as an internal "pH-meter". The same shift of the buffer peak is also seen in the fast MAS spectra. Static ${ }^{31} \mathrm{P}$ powder patterns are consistent with the slow $(1 \mathrm{kHz})$ MAS spectra, i.e. MAS spectra spinning sideband peaks can be calculated from the powder pattern. The MAS spectra provide improved resolution of isotropic chemical shifts.

At least two conclusions can be drawn from the data in Figure 2.3. First, static spectra indicate that the residual shielding anisotropy is axially symmetric. Since the static shielding is non-axial, this requires that TOCL molecules undergo axially symmetric motion about the bilayer normal. Second, the fact that a single shielding tensor in powder patterns and a single lipid peak in fast (6.5 $\mathrm{kHz})$ MAS spectra are observed shows equivalency of the two phosphates in the headgroup of TOCL. 


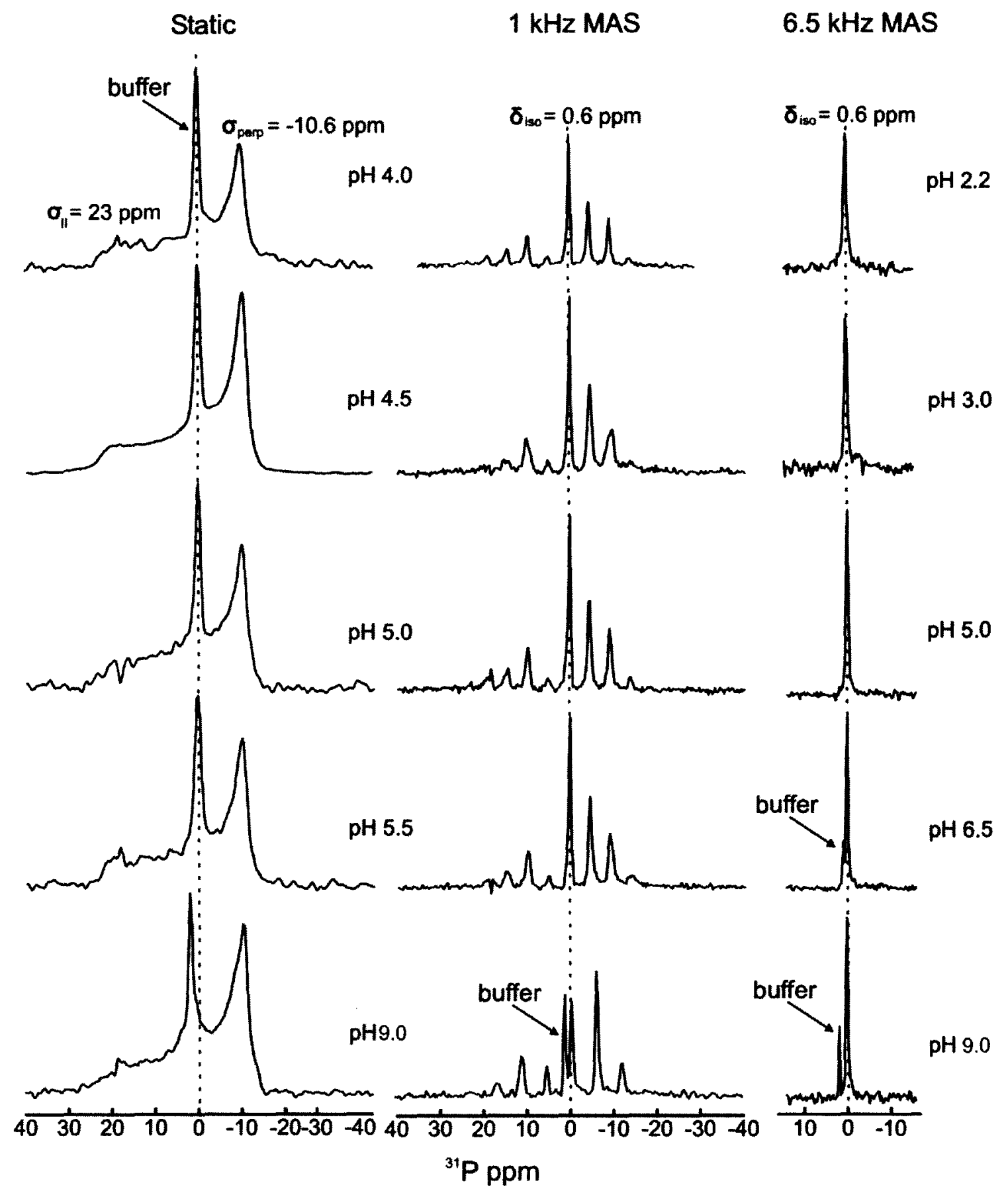

Figure 2.3. Static and MAS ${ }^{31} \mathrm{P}$ NMR spectra of TOCL dispersions in phosphate/citrate phosphate buffer at various $\mathrm{pH}$. 


\section{${ }^{31} \mathrm{P}$ CSA and mobility of the headgroup}

The anisotropy of the ${ }^{31} \mathrm{P}$ shielding tensor depends on the structure and motion of the headgroup. Therefore, conformational changes and/or restrictions of mobility of the headgroup lead to observable changes in ${ }^{31} \mathrm{P}$ CSA. It is known that the headgroup of phosphatidylcholine (PC) is flexible and it reorients around the bilayer normal. Compared to the ${ }^{31} \mathrm{P}$ anisotropy of liquid crystalline phase $\mathrm{PC}$, $45 \mathrm{ppm}$ [80], spectra of lamellar TOCL, Figure 2.3, show smaller ${ }^{31} \mathrm{P}$ anisotropy, about $34 \mathrm{ppm}$. Therefore, the headgroup of $\mathrm{CL}$ is somewhat more flexible than that of PC. Moreover, whereas some lipids have $\mathrm{pH}$ dependent anisotropy [81], TOCL shows no significant change in CSA over the $\mathrm{pH}$ range from 4 to 9 .

\section{${ }^{1} \mathrm{H}$ Search for a protonated phosphate}

Haines and co-workers suggested [72] that their observation of an unusually high $\mathrm{pK}_{\mathrm{a}}$ is a consequence of the formation of hydrogen bond between the central glycerol hydroxyl group and a protonated phosphate from a PA moiety. Acid protons stabilized in a hydrogen bond have characteristic chemical shifts (>10 ppm) that are well downfield of "regular" proton shifts. In principle, such protons can be observed with ${ }^{1} H$ NMR due to their unusual chemical shift. For example, formation of strong hydrogen bonds in the active sites of serine proteases has been suggested [82] based on downfield chemical shifts in ${ }^{1} \mathrm{H}$ NMR. Figure 2.4 shows an example of a proton trapped in the intramolecular hydrogen bond in sodium hydrogen maleate $(\mathrm{NaHM})$. The lower ${ }^{1} \mathrm{H}$ spectrum is $\mathrm{NaHM}$ trihydrate dissolved in DMSO and the top spectrum was obtained after a 
small amount of water was added. The peaks at $\sim 2.5,3.5$ and $\sim 6 \mathrm{ppm}$ are from residual solvent, water and maleate, respectively. The acid-anion trapped proton is at $\sim 20 \mathrm{ppm}$. It, however disappears when a small amount of water is added, presumably due to rapid exchange with solvent water $(\sim 3.7 \mathrm{ppm})$. We conclude that in aqueous samples, trapped protons are not necessarily observable by NMR.

Figure 2.5 shows ${ }^{1} \mathrm{H}$ spectra of TOCL at $\mathrm{pH} 6.5$ and 9.0. The only peak observed in the downfield region below $20 \mathrm{ppm}$ corresponds to the spinning sideband of the lipid peak. This was confirmed by changing the spinning speed $\left(v_{\text {rot }}\right)$. In addition, ${ }^{1} \mathrm{H}$ lipid chemical shifts are the same at $\mathrm{pH} 6.5$ and 9.0. These results provide negative evidence for the formation of the proposed CL cyclic, acid-anion structure within the headgroup. However, as seen in hydrogen maleate example, the downfield peak of the stabilized proton can disappear due to fast exchange. Therefore, we next used ${ }^{13} \mathrm{C}$ NMR to search for evidence of ionizable groups in $\mathrm{CL}$ with physiologically interesting $\mathrm{pK}_{\mathrm{a}}$ 's. ${ }^{13} \mathrm{C}$ spectroscopy is a sensitive monitor of local ionizable groups when charge changes upon titration. 


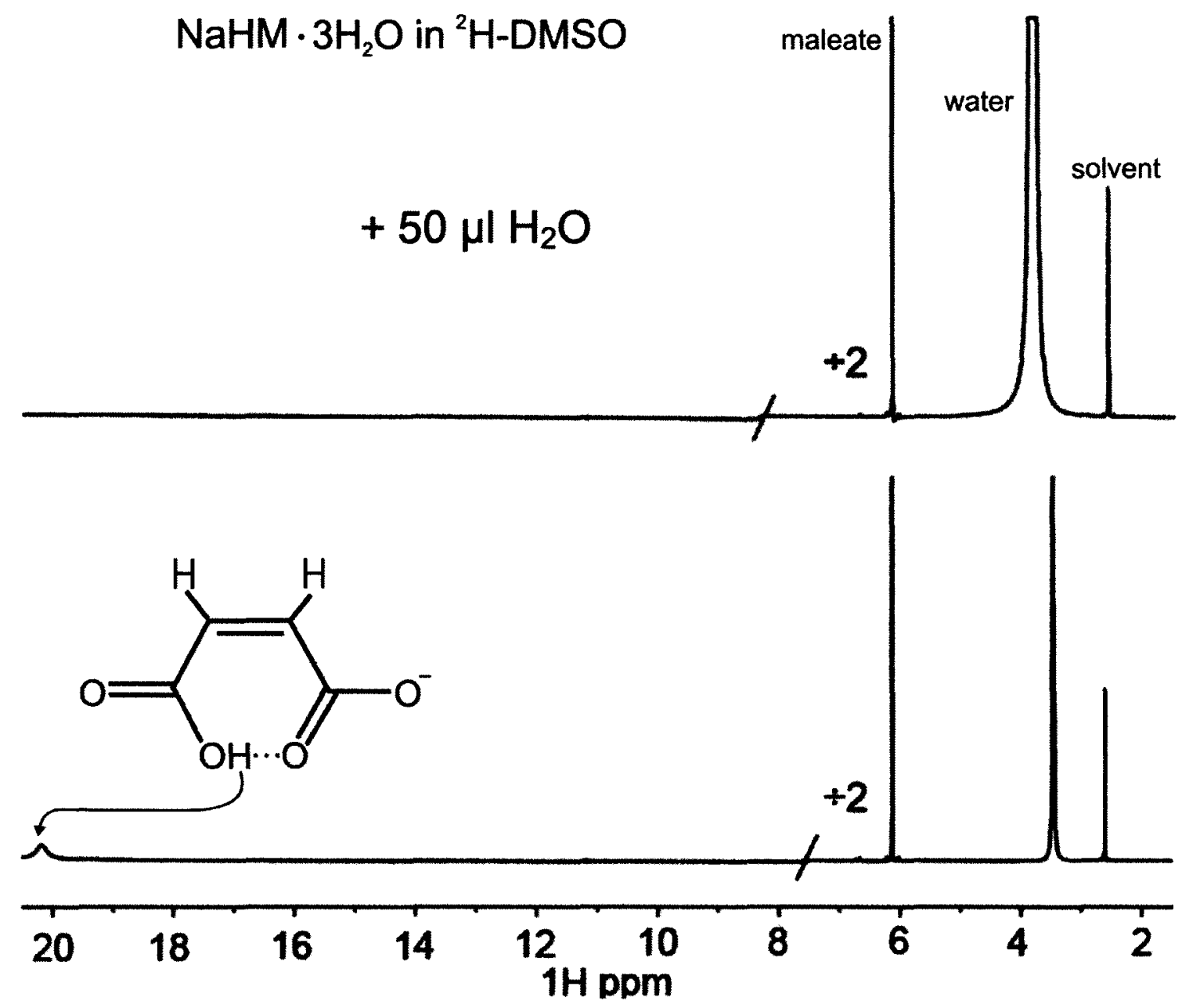

Figure 2.4. ${ }^{1} \mathrm{H}$ spectra of sodium hydrogen $+50 \mu \mathrm{l} \mathrm{H}_{2} \mathrm{O}$ maleate. 

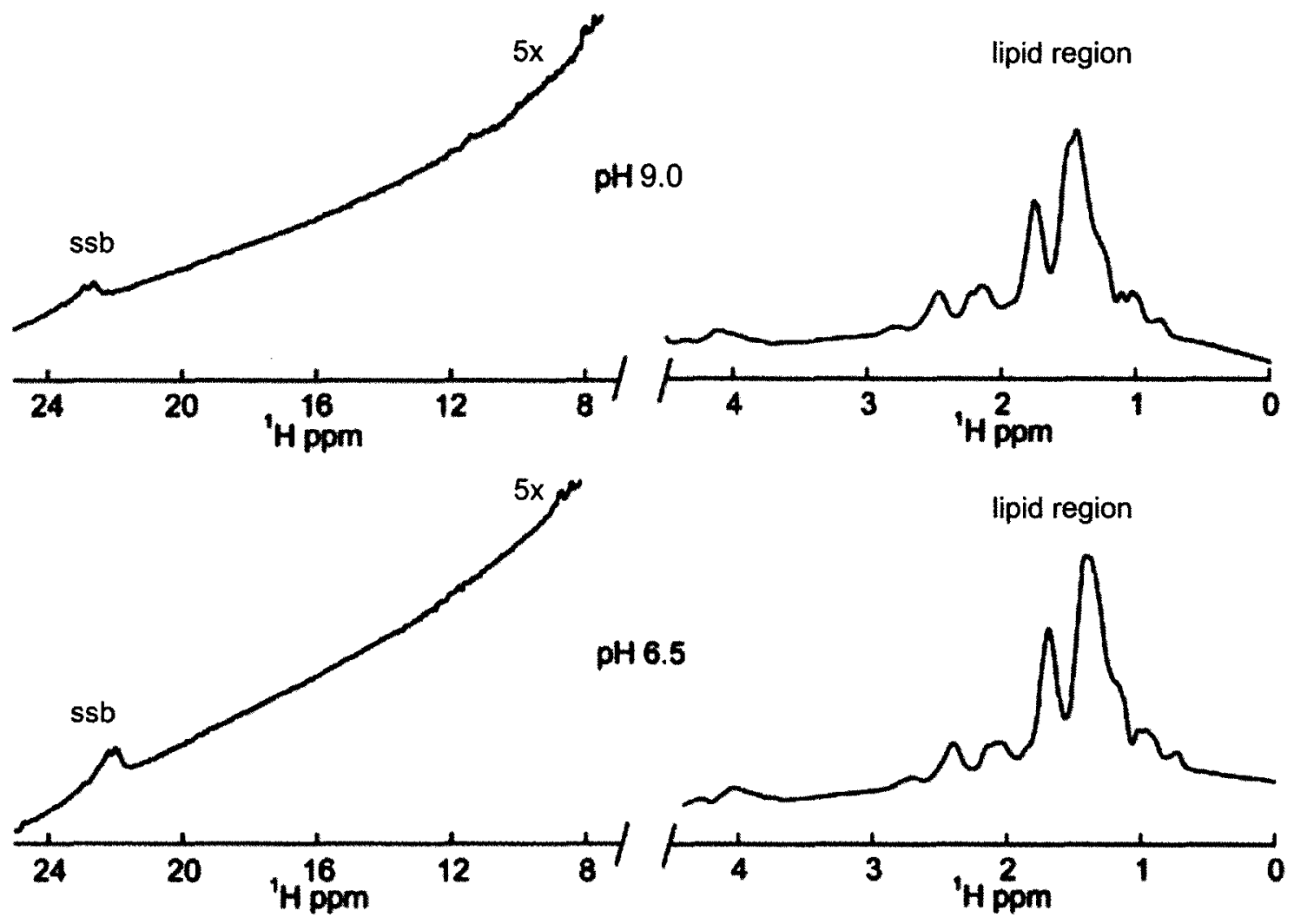

Figure 2.5. ${ }^{1} \mathrm{H}$ MAS spectra of TOCL at $\mathrm{pH} 6.5\left(\mathrm{v}_{\mathrm{rot}}=10 \mathrm{kHz}\right)$ and 9.0 $\left(\mathrm{v}_{\text {rot }}=10.3 \mathrm{kHz}\right)$.Spectra are broken into 2 sections: the lipid region (0 to $\left.4 \mathrm{ppm}\right)$ and the downfield region where the acid-anion proton is expected ( $>10 \mathrm{ppm}$ ). The signals below $20 \mathrm{ppm}$ are spinning sidebands of the lipid. The downfield sections are vertically expanded 5 -fold compared to the upfield sections. 


\section{Assignment of ${ }^{13} \mathrm{C}$ and ${ }^{1} \mathrm{H}$ NMR spectra}

${ }^{13} \mathrm{C}$ MAS NMR spectra with good $\mathrm{S} / \mathrm{N}$ are obtained for TOCL over the $\mathrm{pH}$ range from 4.5 to 9.0 . The ${ }^{13} \mathrm{C}$ spectrum (Figure 2.6) has distinct regions as labeled. For our purpose, the most interesting region of the spectrum is the glycerol region with five resolved peaks. If the cyclic structure with trapped proton forms as suggested by Haines [72], the headgroup glycerol carbons are expected to have $\mathrm{pH}$-dependent chemical shifts.

A schematic of the TOCL structure with nomenclature used for the assignment is shown on Figure 2.7. $G_{1}, G_{2}$ and $G_{3}$ are $C$ atoms at position 1, 2 and 3 of the side PA "glycerols"; $G_{1}^{\prime}$ are at equivalent positions 1,3 and $G_{2}^{\prime}$ is at position 2 of the central "glycerol". Atoms, carbons and protons, from 1 to 18 are from the fatty acyl chains with the double bonds at the indicated positions.



Figure 2.6. ${ }^{13} \mathrm{C}$ MAS NMR spectrum of TOCL in phosphate buffer at $\mathrm{pH} 7.0$. 


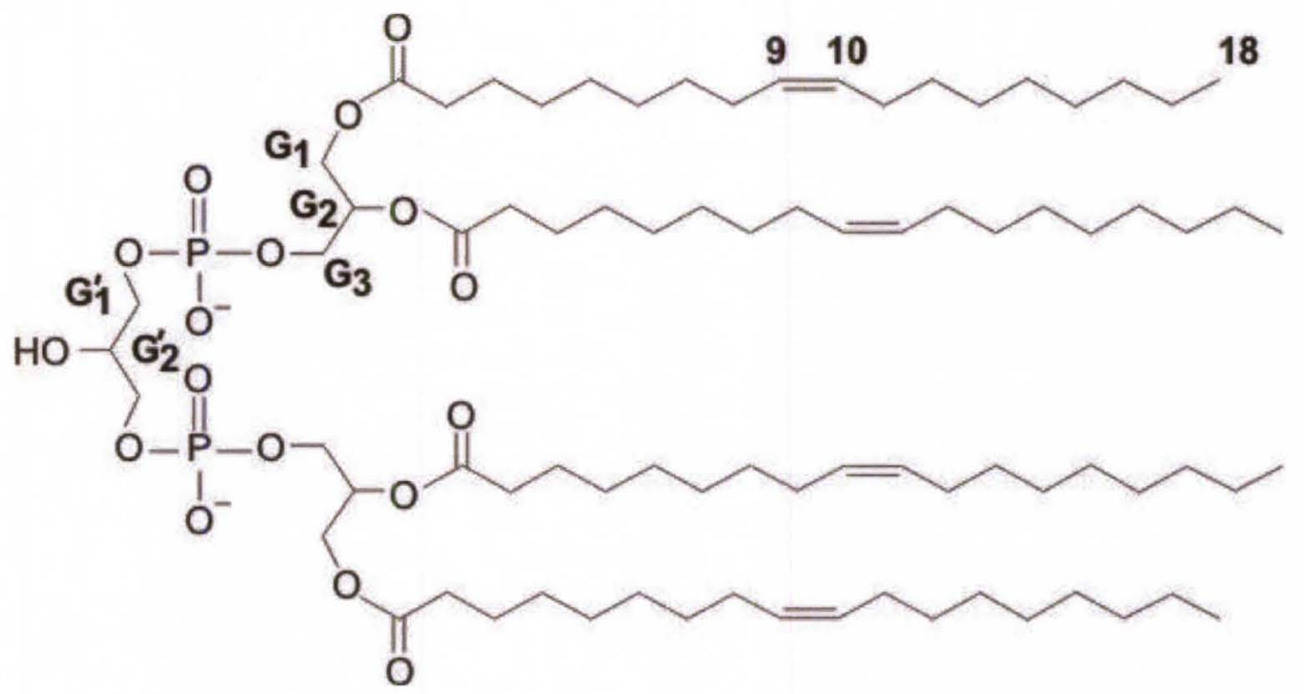

Figure 2.7. Schematic structure of TOCL and nomenclature used for the assignment of ${ }^{13} \mathrm{C}$ and ${ }^{1} \mathrm{H}$ NMR spectra.

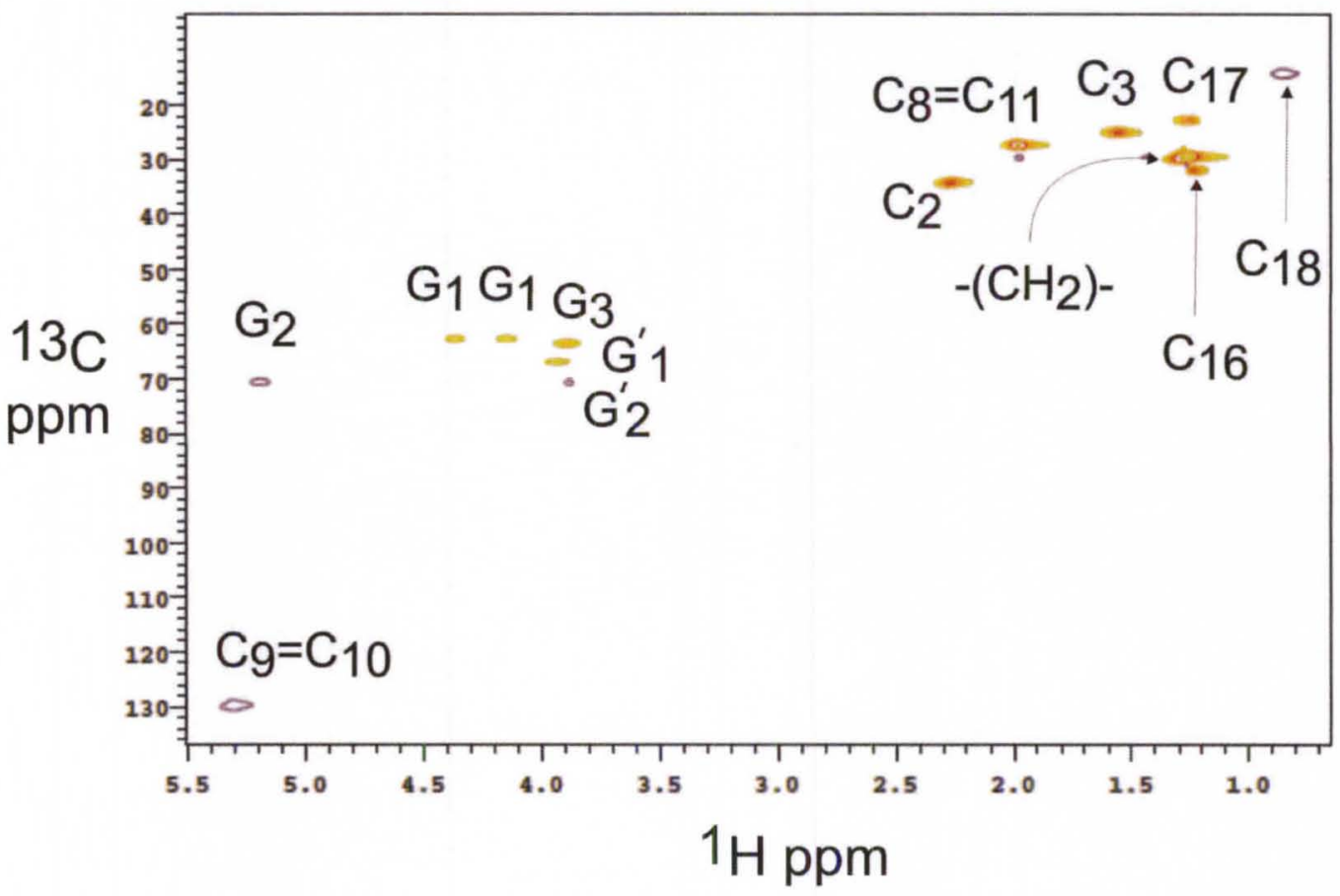

Figure 2.8. HSQC spectrum of $\mathrm{TOCL}$ in $\mathrm{CDCl}_{3}$. 
The strategy used to assign the closely spaced glycerol region of spectra in Figure 2.9 and Figure 2.10 is to correlate ${ }^{13} \mathrm{C}$ and ${ }^{1} \mathrm{H}$ shifts using the HSQC experiment (Figure 2.8). This approach is possible since chemical shifts observed in MAS spectra of the $L_{\alpha}$ phase $C L$ dispersions in phosphate buffer are essentially the same as when the lipid is dissolved in deuterated chloroform. In the HSQC spectrum, negative (purple) peaks for methine $\left(G_{2}\right.$ and $\left.G_{2}^{\prime}\right)$ carbons at $70.3 \mathrm{ppm}$ and $71.4 \mathrm{ppm}$ are readily distinguished from the positive (yellow) methylene signals $\left(G_{1}, G_{3}\right.$ and $\left.G_{1}^{\prime}\right)$. On the basis of signal intensity, the smaller methine peak at $70.3 \mathrm{ppm}$ is assigned to $\mathrm{G}_{2}^{\prime}(1$ carbon) and the $\sim 2$-fold larger signal at $71.4 \mathrm{ppm}$ to $\mathrm{G}_{2}$ (2 carbons). Acylation at glycerol methylene carbons induces inequivalency of the attached protons, consequently the ${ }^{1} \mathrm{H}$ split signals with a ${ }^{13} \mathrm{C}$ shift of $63.7 \mathrm{ppm}$ are assigned to $\mathrm{G}_{1}$. The remaining methylene signals, $64.4 \mathrm{ppm}$ and $67.2 \mathrm{ppm}$, are assigned to $\mathrm{G}_{3}\left(2\right.$ carbons) and $\mathrm{G}_{1}^{\prime}$ (2 carbons). Based on ${ }^{13} \mathrm{C}$ shifts observed in lecithins [78] the $64.4 \mathrm{ppm}$ signal is tentatively assigned to $\mathrm{G}_{3}$.

Remaining signals are due to the acyl chains. The most downfield signals are from fatty acid carbonyl groups, $173.51 \mathrm{ppm}$ (in $\mathrm{CDCl}_{3}$ ), and the unsaturated carbons $\left(\mathrm{C}_{9}\right.$ and $\left.\mathrm{C}_{10}\right)$ of the fatty acid chains at $129.60 \mathrm{ppm}$. Assignments in the aliphatic region were based on the known assignments of DMPC [83] and fatty acid esters. Complete listing of these results is given in Figure 2.9, Figure 2.10 and in the Table 2.1. 


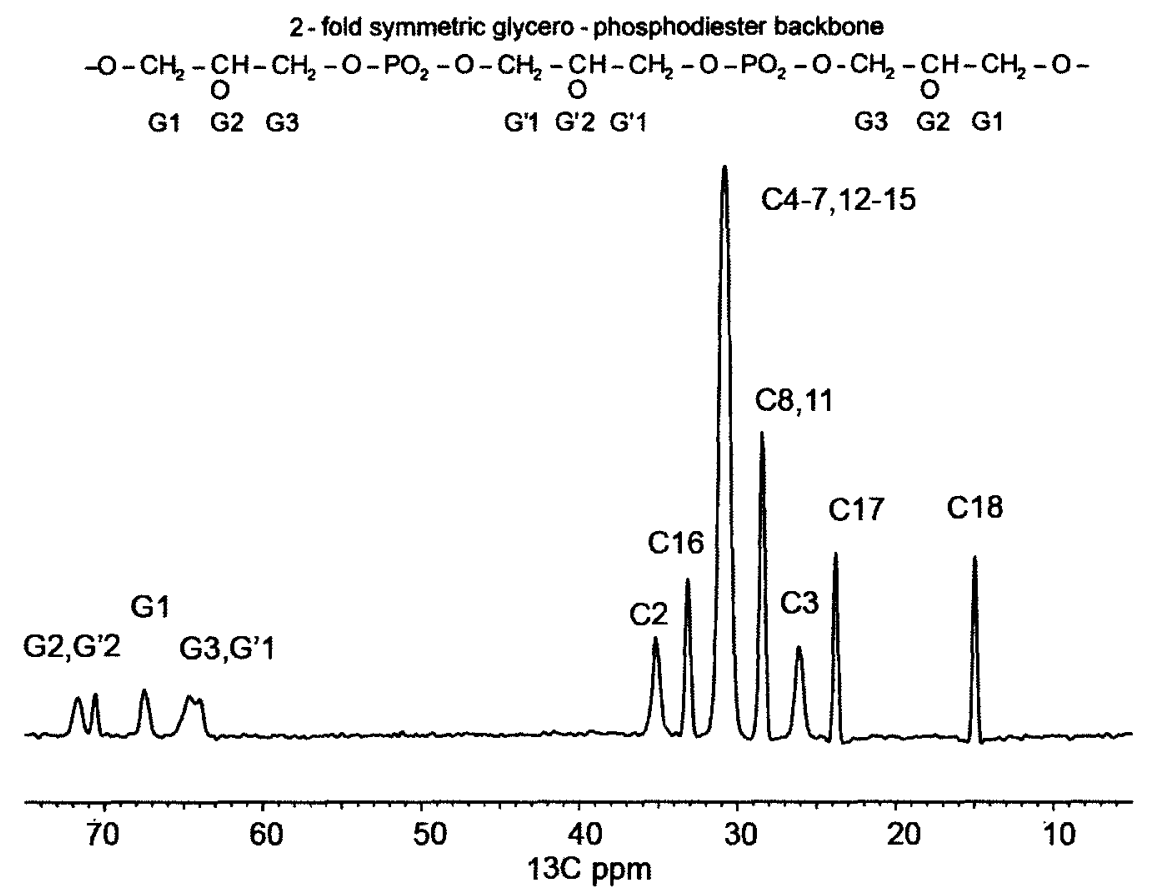

Figure 2.9. ${ }^{13} \mathrm{C}$ MAS NMR spectrum of TOCL in phosphate buffer.

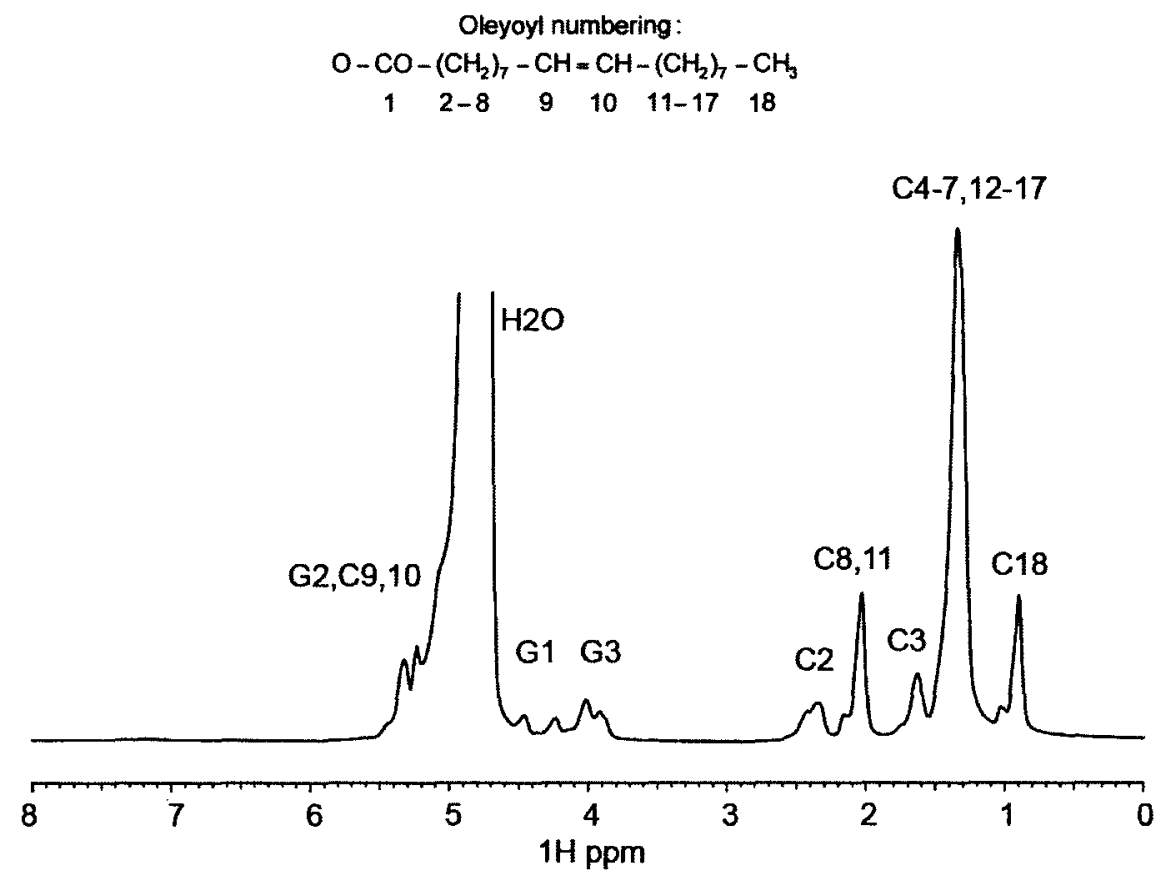

Figure 2.10. ${ }^{1} \mathrm{H}$ MAS NMR spectrum of TOCL in phosphate buffer. 
Table 2.1. Assignments of ${ }^{1} \mathrm{H}$ and ${ }^{13} \mathrm{C}$ NMR spectra of TOCL.

\begin{tabular}{ccccc}
\hline & \multicolumn{2}{c}{${ }^{13} \mathrm{C}$ chemical shift } \\
Atom position & $\mathrm{CDCl}_{3}$ & $\begin{array}{c}100 \mathrm{mM} \mathrm{Na} \\
\text { phosphate } \\
\text { buffer, } \mathrm{pH} 7.0\end{array}$ & $\mathrm{CDCl}_{3}$ & $\begin{array}{c}{ }^{1} \mathrm{H} \text { chemical shift } \\
100 \mathrm{mM} \mathrm{Na} \\
\text { phosphate } \\
\text { buffer, } \mathrm{pH} 7.0\end{array}$ \\
\hline 1 & & 174.4 & - & - \\
2 & 173.51 & 34.8 & 2.22 & 2.3 \\
3 & 34.06 & 25.8 & 1.51 & 1.6 \\
9,10 & 24.86 & 130.2 & 5.26 & 5.3 \\
8,11 & 129.60 & 28.1 & 1.93 & 2.0 \\
$4-7,12-15$ & 27.22 & 30.4 & 1.24 & 1.3 \\
16 & 29.50 & 32.8 & 1.19 & 1.3 \\
17 & 31.89 & 23.5 & 1.20 & 1.3 \\
18 & 22.65 & 14.7 & 0.90 & 0.8 \\
$\mathrm{G}_{1}$ & 14.10 & 63.7 & $4.12,4.34$ & $4.2,4.5$ \\
$\mathrm{G}_{1}^{\prime}$ & 62.85 & 67.2 & 3.91 & $3.9-4.0$ \\
$\mathrm{G}_{2}$ & 67.23 & 71.4 & 5.17 & 5.2 \\
$\mathrm{G}_{2}^{\prime}$ & 69.51 & 70.3 & 3.87 & $3.9-4.0$ \\
$\mathrm{G}_{3}$ & 63.41 & 64.4 & 3.87 & $3.9-4.0$ \\
\hline
\end{tabular}


NMR titration: ${ }^{13} \mathrm{C}$ and ${ }^{31} \mathrm{P}$ chemical shifts vs. $\mathrm{pH}$

${ }^{13} \mathrm{C}$ chemical shifts of TOCL from $\mathrm{pH} 4.5$ to 9.0 are presented in Figure 2.11. We observe that chemical shifts are constant within experimental error, $\pm 0.1 \mathrm{ppm}$, over this physiologically important range.

(a)
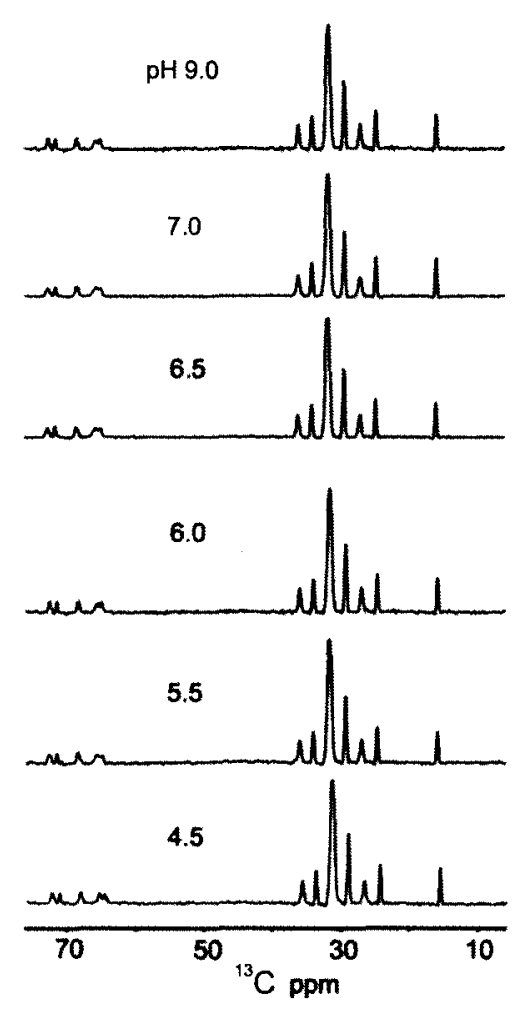

(b)

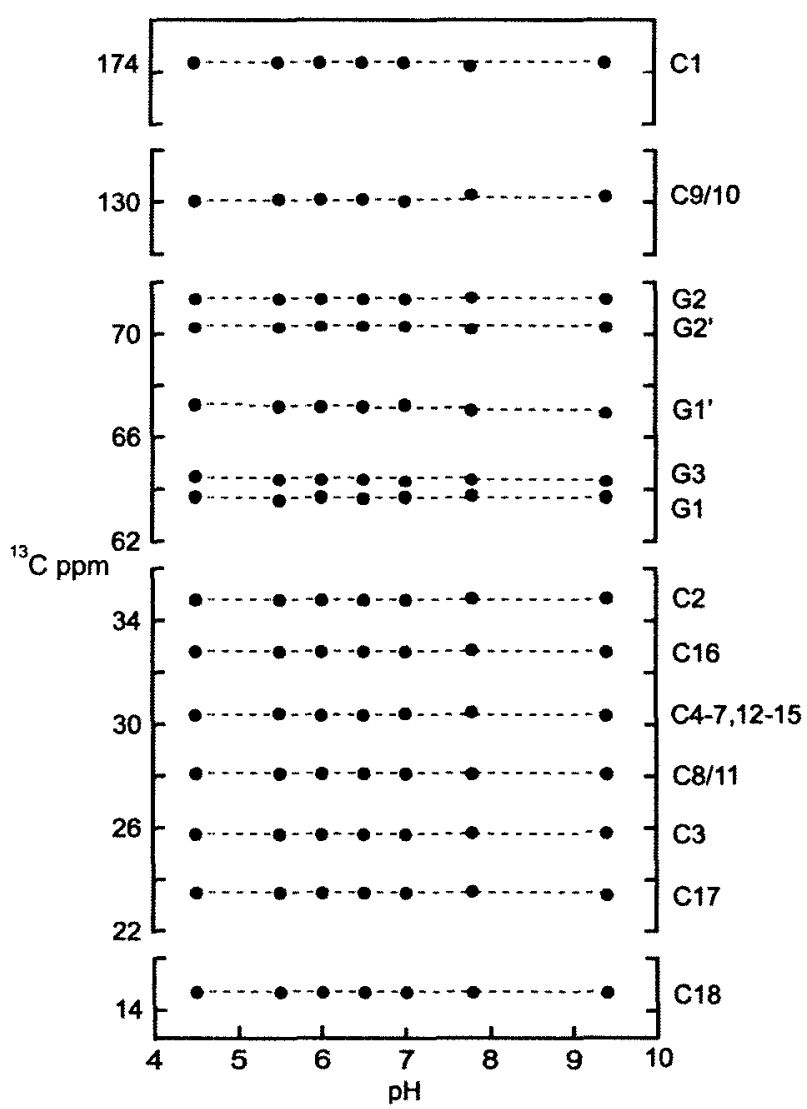

Figure 2.11. ${ }^{13} \mathrm{C}$ MAS NMR spectra of TOCL in phosphate buffer (a) and site specific chemical shifts (b).

Similarly, Figure 2.12 and Figure 2.13 show that ${ }^{31} \mathrm{P}$ chemical shifts of TOCL are also independent of $\mathrm{pH}$. Note that in these spectra, the buffer signal moves downfield with increased $\mathrm{pH}$. 



Figure 2.12. ${ }^{31} \mathrm{P}$ MAS NMR spectra of TOCL.



Figure 2.13. ${ }^{31} \mathrm{P}$ chemical shifts of TOCL $(\bullet)$ and phosphate/citrate phosphate buffer $(x)$ as function of $\mathrm{pH}$. 
It is instructive to compare the behavior of phosphoric acid when it is titrated and observed by NMR with the behavior of Phosphodiesters, such as CL. This comparison is appropriate since both phosphoric acid and phosphodiester are neutral and have similar $\mathrm{pK}_{\mathrm{a}}$ values (Figure 2.14). The results of an NMR titration of phosphoric acid are shown in Figure 2.15.

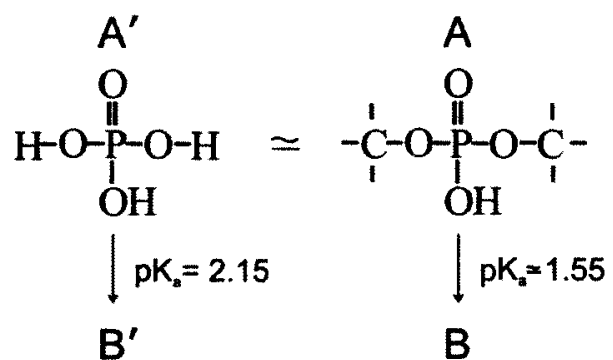

Figure 2.14. Analogy between phosphoric acid and phosphodiester.

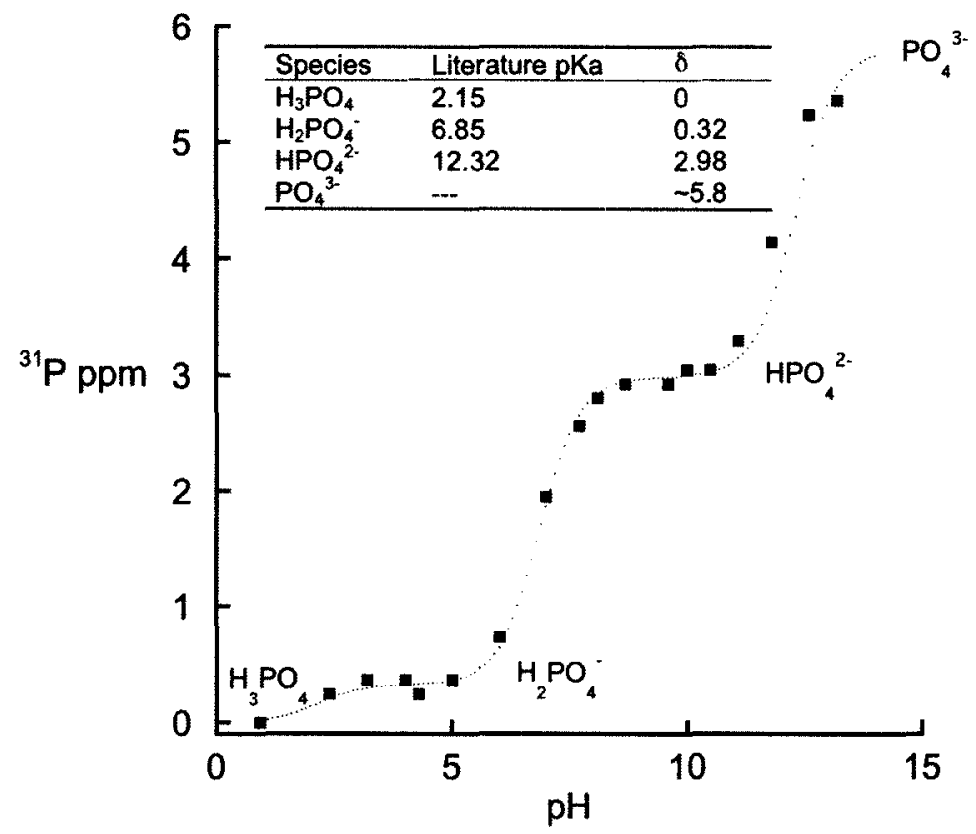

Figure 2.15. NMR titration curve for $\mathrm{H}_{3} \mathrm{PO}_{4}$. $\mathrm{pK}_{\mathrm{a}}$ values are from [84].

The change in chemical shift upon deprotonation of phosphoric acid, $\mathrm{H}_{3} \mathrm{PO}_{4}$, is $0.32 \mathrm{ppm}$ and we expect a similarly small change for deprotonation of 
phosphodiesters like CL. While ${ }^{31} \mathrm{P}$ is not a particularly sensitive probe for this, our experiments have enough resolution to see such small changes and we did not observe any change in ${ }^{31} \mathrm{P}$ shifts with titration.

\subsection{Discussion and conclusions}

Well resolved ${ }^{13} \mathrm{C}$ and ${ }^{31} \mathrm{P}$ MAS NMR spectra of TOCL show $\mathrm{pH}$ independent chemical shifts and indicate no titration over the physiologically relevant $\mathrm{pH}$ range tested. Moreover, ${ }^{1} \mathrm{H}$ MAS NMR spectra show no peak for an acid proton stabilized by a hydrogen bond. Thus, we conclude that the structural hypothesis for the CL's headgroup in which phosphates form a tight bicyclic structure $\mathrm{H}$-bonding to the hydroxyl group of the connecting glycerol and trap a proton from the environment [72] is incorrect.

To appreciate this conclusion, we estimate the $\mathrm{H}$-bond energy required to raise a phosphodiester $\mathrm{pK}_{\mathrm{a}}$ from a normal value, $\mathrm{pK}_{\mathrm{a}} \sim 1.5$, to a value that would make it a proton storage trap at physiological $\mathrm{pH}$ values, $\mathrm{pK}_{\mathrm{a}} \sim 7.5$. To do this, consider the thermodynamic cycle (Figure 2.16 ) in which the singly protonated cardiolipin, $\mathrm{CLH}$, can either lose a proton directly with $\mathrm{pK}_{\mathrm{a} 1}$ or form a stabilizing bond, CLH---B, with free energy change $\Delta \mathrm{G}_{\text {stab }}<0$.



Figure 2.16. Model for the estimation of energy of stabilizing bond. 
Subsequently, $\mathrm{CLH}---\mathrm{B}$, can lose its proton with $\mathrm{pK}_{\mathrm{a} 2}>\mathrm{pK}_{\mathrm{a} 1}$. This cycle shows that the $\mathrm{pK}_{\mathrm{a}}$ 's and $\Delta \mathrm{G}_{\mathrm{stab}}$ are related by

$$
\Delta \mathrm{G}_{\mathrm{stab}}+\Delta \mathrm{G}_{2}=\Delta \mathrm{G}_{1}
$$

or

$$
\Delta p K_{\mathrm{a}}=p K_{\mathrm{a} 2}-p K_{\mathrm{a} 1}=-\Delta \mathrm{G}_{\mathrm{stab}} /(2.303 R \mathrm{R}) \sim-\Delta \mathrm{G}_{\mathrm{stab}} /(5.8 \mathrm{~kJ} / \mathrm{mol}) .
$$

If the stabilizing interaction is a hydrogen bond, as suggested in [72], then $\left|\Delta G_{\text {stab }}\right|$ is not more than $12 \mathrm{~kJ} / \mathrm{mol}$ and, consequently, $\Delta p K a$ is not more than 2 . To stabilize a phosphodiester, $\mathrm{pK}_{\mathrm{a} 1} \sim 1.5$, so that its $\mathrm{pK}_{\mathrm{a} 2}$ is increased to 7.5 , would require stabilization in excess of $35 \mathrm{~kJ} / \mathrm{mol}$. We view this as unrealistic. We conclude that there is no evidence or chemical basis that lamellar CL possesses a titratable group with a $\mathrm{pK}_{\mathrm{a}}$ in the range of 4 to 9 .

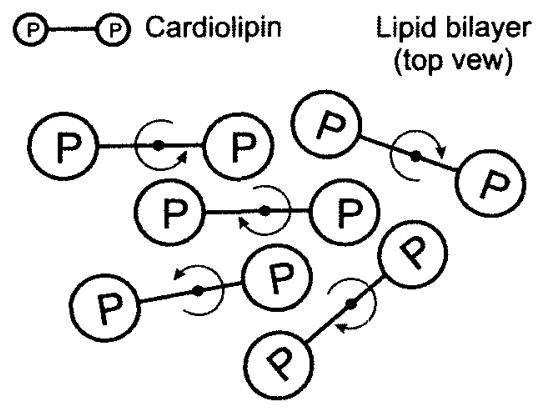

Figure 2.17. Model for the axially symmetric reorientation of $\mathrm{CL}$ within the bilayer.

Our results also show that the ${ }^{31} \mathrm{P}$ CSA of the TOCL headgroup is axially symmetric and the anisotropy is less than that of PC. We conclude that it is less ordered than the flexible headgroup of PC and suggest the following model for lamellar cardiolipin membranes (Figure 2.17). Headgroups within a CL molecule are flexibly connected and each $\mathrm{CL}$ molecule reorients with axially symmetric motion within the membrane bilayer. 


\section{CHAPTER 3}

\section{${ }^{2} \mathrm{H}$ MULTIPLE QUANTUM NMR}

\subsection{Introduction}

The advent of two-dimensional Fourier transform spectroscopy [85-86] triggered the development of new NMR fields: homo- and heteronuclear correlation spectroscopy and multiple quantum (MQ) spectroscopy [86-87]. The availability of the second dimension is essential for $M Q$ spectroscopy, since coherent MQ oscillations of spins do not produce magnetic dipole radiation [88], i.e. they induce no signal and can only be detected indirectly after conversion to observable single quantum (1Q) coherence. At the initial stage of development, MQ spectroscopy dealt with improvement of spectral resolution via elimination of quadrupolar and field inhomogeneity broadening $[86-87,89]$, with relaxation time measurements [90-92], and with detection of couplings [93-94]. The spectrum of applications continues and is now used in measurements of dipolar couplings [95-97], detection of transient ordering in gases [98] and fluids [99-100], magnetic resonance imaging [101-105], and MQ diffusion studies [106-107] etc.

In spite of the diversity of applications, the pulse sequences employed in these experiments underwent only modest changes. The initial three-pulse experiment was supplemented with refocusing pulses and with 16-step phase 
cycles for signal filtration. Even the most recent studies exploit relatively simple pulse sequences and phase cycles. In some cases it suffices, but in others it leads to partial cancellation of the desired signals that have opposite phases. This leads to dispersive signals and poor $\mathrm{S} / \mathrm{N}$ in general.

In this study, we have designed a more general pulse sequence and phase cycle. The ultimate goal is to address the mechanism of elasticity in elastin by observing the solvent part of an elastin sample. The solvent is potentially ordered on the surface of the elastin fiber, and our task is to detect the amount of ordered solvent, while suppressing the signal from bulk, isotropically reorienting solvent. This is relevant to an alternative hypothesis about the mechanism of elasticity that is based on the hydrophobic effect. MQ spectroscopy is useful in this case because it allows one to distinguish ordered, anisotropically reorienting water, from the bulk, isotropic water. MQ NMR has been used for solvent ordering measurements in a variety of systems, including purple membrane suspensions [99], polymer membranes like Nafion [100], biological samples such as blood vessels [105], cartilage [104], spinal disk tissue [108-109], collagen [103, 110-111], and elastin [112-113]. We have improved on the pulse sequence commonly used, and devised a method for analysis of the results that allows us to estimate the amount of ordered water in the sample and the strength of the residual quadrupolar coupling, i.e., the degree to which the water is ordered at the protein surface.

In this chapter, I present background information on MQ spectroscopy, a description of the development of a new pulse sequence and phase cycle, 
experimental verification of the sequence, and the method for analysis of the results. Methods described in this theoretical chapter are then used for the study of the mechanism of elastin's elasticity, described in the following chapter. The approach is general and can be used to study solvent ordering in other systems.

\subsection{MQ spectroscopy background}

\section{Density operator}

The state of a quantum system is described by the wavefunction, $\psi$, that can be expressed as a linear combination of eigenstates:

$$
\Psi=|\Psi\rangle=a_{1}\left|\varphi_{1}\right\rangle+a_{2}\left|\varphi_{2}\right\rangle+\ldots=\sum_{i} a_{i}\left|\varphi_{i}\right\rangle
$$

with coefficients $a_{i}=\left\langle\varphi_{i} \mid \psi\right\rangle$. The basis functions, $\left|\varphi_{i}\right\rangle$, are orthogonal $\left\langle\varphi_{i} \mid \varphi_{j}\right\rangle=0$ and normalized $\left\langle\varphi_{i} \mid \varphi_{i}\right\rangle=1$, and the wavefunction, $\psi$, is a vector:

$$
\Psi=\left(\begin{array}{c}
a_{1} \\
a_{2} \\
\vdots
\end{array}\right)
$$

Evolution of the wavefunction is governed by the time-dependent Schrödinger equation,

$$
H|\Psi\rangle=i \frac{\partial|\psi\rangle}{\partial t},
$$

where $\hbar$ is set to 1 , and $\mathrm{H}$ is the interaction Hamiltonian. In the case of a timeindependent Hamiltonian, the solution is 


$$
|\Psi(t)\rangle=e^{-i H t}|\Psi(0)\rangle
$$

To make $\mathrm{H}$ time-independent, we use rotating frame Hamiltonians that remove the high frequency Larmor precession, $\omega_{0}$, resulting from the large Zeeman interaction. With this, peak positions have frequency $\omega_{0}-\omega_{\mathrm{RF}}$ that are defined relative to the center of the spectrum, $\omega_{\mathrm{RF}}$. For our purposes, there are three relevant spin Hamiltonians. Two of these, offset and quadrupolar, are internal, $\mathrm{H}_{\mathrm{os}}$ and $\mathrm{H}_{\mathrm{q}}$, and are the energies of the nuclear spin interacting with the surrounding electrons. The third Hamiltonian, $H_{R F}$, is for the applied resonant RF field that the spectroscopist uses to manipulate the spin system.

$$
\begin{gathered}
H_{o s}=-\Delta \omega_{o s} I_{z}, \\
H_{q}=\frac{1}{2 I+1} \omega_{q}\left(3 l_{z}^{2}-I(I+1)\right), \\
H_{R F}=-\omega_{1} l_{k},
\end{gathered}
$$

where $I_{k}(k=x, y, z)$ is the $k$-component of spin angular momentum, $I$ is a spin quantum number, $\Delta \omega_{o s}=\omega_{0}-\omega_{R F}$ is an offset frequency, $\omega_{q}$ is a quadrupolar coupling, and (as we shall see) an RF pulse of duration $t$ and strength $\mathrm{\gamma B}_{1}$ rotates $1 Q$ coherence through an angle $\theta=\omega_{1} t$ about the $k$-axis. Since the Zeeman and chemical shielding interactions depend in the same way on spin variables, $\Delta \omega_{o s}$ contains the effect of chemical shielding and the small difference between a spin's precession frequency and the rotating frame frequency. In solids and semisolids, chemical shielding is spatially anisotropic, so $\Delta \omega_{\text {os }}$ depends on molecular orientation. 
In quantum mechanics, the expectation value $\langle\Omega\rangle$ of an observable, $\Omega$, is given by

$$
\langle\Omega\rangle=\langle\psi|\Omega| \psi\rangle=\sum_{i, j} a_{i}^{*} a_{j}\left\langle\varphi_{i}|\Omega| \varphi_{j}\right\rangle=\sum_{i, j} a_{i}^{*} a_{j} \Omega_{i j}
$$

The matrix elements $a_{i}^{*} a_{j}$ are tabulated in the form of a Hermitian $(2 l+1) \times(2 l+1)$ matrix, $\rho_{i j}$, which is known as the density matrix or density operator $\rho$. Therefore, $\langle\Omega\rangle=\sum_{\mathrm{i}, \mathrm{j}} \Omega_{\mathrm{ij}} \rho_{\mathrm{ij}}=\operatorname{Tr}(\Omega \rho)$.

Comparison of the above shows that the density operator is the direct product of the wave vector with itself,

$$
\rho=|\psi\rangle\langle\psi|=\left(\begin{array}{ccc}
a_{1}^{*} a_{1} & a_{1}^{*} a_{2} & \cdots \\
a_{2}^{*} a_{1} & a_{2}^{*} a_{2} & \cdots \\
\vdots & \vdots & \ddots
\end{array}\right) .
$$

The density operator contains all information about the dynamics of the spin system. Elements of the density matrix have clear physical meaning: the diagonal element $\rho_{i i}$ represents the population of energy level $i$, while the offdiagonal element $\rho_{i j}$ corresponds to the transition between $|i\rangle$ and $|j\rangle$ states.

Time evolution of the density operator is then given by

$$
\begin{aligned}
\frac{\partial \rho}{\partial \mathrm{t}} & =\frac{\partial|\Psi\rangle\langle\psi|}{\partial \mathrm{t}}=\frac{\partial|\Psi\rangle}{\partial \mathrm{t}}\langle\Psi|+| \Psi\rangle \frac{\partial\langle\psi|}{\partial \mathrm{t}} \\
& =-\mathrm{iH}|\Psi\rangle\langle\Psi|+\mathrm{i}| \Psi\rangle\langle\Psi| \mathrm{H}=-\mathrm{iH} \rho+\mathrm{iH} \rho \\
& =-\mathrm{i}[\mathrm{H}, \rho],
\end{aligned}
$$


and is known as the Liouville-von Neumann equation. The solution of this equation, in the case of time-independent, rotating frame Hamiltonians is

$$
\rho(t)=e^{-i H t} \rho(0) e^{i H t},
$$

where $\rho(0)$ describes the spin system at $t=0$.

\section{Single transition operators and coherences}

A logical extension of the analogy between the wavefunction and the density operator is to formulate the density operator as a linear combination of orthogonal and normalized basis operators, $\mathrm{O}_{\mathrm{i}}$ :

$$
\rho=\sum_{i} a_{i} O_{i}
$$

Insofar as the basis operators cover the space, their choice is arbitrary. It is convenient to choose operators with clear physical meaning. With trace orthogonality, the expectation value of each of the basis operators is equal to the corresponding coefficient in the linear combination, $\left\langle\mathrm{O}_{i}\right\rangle=\mathrm{a}_{\mathrm{i}}$.

For ${ }^{2} \mathrm{H}, \mathrm{I}=1$, there are $2 \mathrm{I}+1=3$ possible spin states and the density operator has $3 \times 3=9$ elements (we refer the reader to Appendix A for the similar description of ${ }^{17} \mathrm{O}$ ). Therefore, to represent the density operator as a linear combination of basis operators, a 9 operator basis set is required. We use operators related to the " $x, y$ and $z$ components" of the 3 transitions. These "single transition operators", $\mathrm{l}_{x}^{\mathrm{i}, \mathrm{j}}, \mathrm{l}_{y}^{\mathrm{i}, \mathrm{j}}$ and $\mathrm{l}_{z}^{\mathrm{i}, \mathrm{j}}$, describe selective excitation of transitions between states $|i\rangle$ and $|j\rangle$. These operators are defined as follows [114]: 


$$
\begin{aligned}
& \left\langle i||_{x}^{\mathrm{rs}} \mid \mathrm{j}\right\rangle=\frac{1}{2}\left(\delta_{\mathrm{ir}} \delta_{\mathrm{js}}+\delta_{\mathrm{is}} \delta_{\mathrm{jr}}\right) \\
& \left\langle i||_{\mathrm{y}}^{\mathrm{rs}} \mid \mathrm{j}\right\rangle=\frac{i}{2}\left(-\delta_{\mathrm{ir}} \delta_{\mathrm{js}}+\delta_{i s} \delta_{\mathrm{jr}}\right) \\
& \left\langle i||_{\mathrm{z}}^{\mathrm{s}} \mid \mathrm{j}\right\rangle=\frac{1}{2}\left(\delta_{\mathrm{ir}} \delta_{\mathrm{jr}}-\delta_{\mathrm{is}} \delta_{\mathrm{js}}\right)
\end{aligned}
$$

The $\mathrm{z}$-component operators, $\mathrm{l}_{\mathrm{z}}^{-1,0}, \mathrm{l}_{\mathrm{z}}^{0,1}$ and $\mathrm{I}_{\mathrm{z}}^{-1,1}$, correspond to level populations and $\mathrm{I}_{z}^{-1,0}+\mathrm{I}_{z}^{0,1}=\mathrm{I}_{z}^{-1,1} \quad\left(\mathrm{I}_{z}^{\mathrm{i}, \mathrm{j}}\right.$ are not linearly independent). The "transverse" components, $l_{k}^{i, j}$ (where $k=x, y$ ), are known as coherences and are closely related to the transverse magnetizations of the allowed transitions, $I_{y}=l_{y}^{-1,0}+l_{y}^{0,1}$ and $I_{x}=I_{x}^{-1,0}+I_{x}^{0,1}$. A coherence between two eigenstates $|i\rangle$ and $|j\rangle$ is non-zero when $\rho_{i j}(t)=a_{i}^{*} a_{j}$ does not vanish [115]. Each eigenstate is characterized by magnetic quantum number $\mathrm{m}$. The change in magnetic quantum number, $\Delta \mathrm{m}$, is called the order of the coherence. Single quantum coherences $(\Delta m= \pm 1)$ are the observable transverse magnetizations, and multiple quantum coherences $(\Delta m \neq \pm 1)$ for $\mathrm{I}=1$ are $\mathrm{I}_{x}^{-1,1}$ and $\mathrm{I}_{y}^{-1,1}$. Explicit $3 \times 3$ matrices for the 9 basis operators of ${ }^{2} \mathrm{H}$ are given in Table 3.1 and the Octave script for their calculation is given in Appendix B. 
Table 3.1. Matrix representations of single transition operators, $\mathrm{l}_{\mathrm{k}}^{\mathrm{ij}}$, for $\mathrm{I}=1$.

\begin{tabular}{|c|c|c|c|c|c|c|c|c|c|}
\hline $\mathrm{i} \rightarrow \mathrm{j}$ & & $x=x$ & & & $k=y$ & & & $k=2$ & \\
\hline \multirow{3}{*}{$-1 \rightarrow 0$} & 0 & $1 / 2$ & $0)$ & 0 & $-i /$ & $0)$ & $(1 / 2$ & 0 & $0)$ \\
\hline & $1 / 2$ & 0 & 0 & $\mathrm{i} / 2$ & 0 & 0 & 0 & -1 & 120 \\
\hline & 0 & 0 & $0)$ & 0 & 0 & 0) & 0 & 0 & 0) \\
\hline \multirow{3}{*}{$0 \rightarrow 1$} & 10 & 0 & 0 & $(0$ & 0 & 0 & $(0$ & 0 & 0 \\
\hline & 0 & & $1 / 2$ & 0 & 0 & $-i / 2$ & & $1 / 2$ & 0 \\
\hline & $\begin{array}{ll}0 & 1\end{array}$ & 12 & 0 & 0 & 12 & 0 & 0 & 0 & $-1 / 2$ \\
\hline \multirow{3}{*}{$-1 \rightarrow 1$} & 0 & 0 & $1 / 2)$ & 0 & 0 & $-i / 2)$ & $(1 / 2$ & 0 & 0 \\
\hline & 0 & 0 & 0 & 0 & 0 & 0 & 0 & 0 & 0 \\
\hline & $1 / 2$ & 0 & 0 & $(i / 2$ & 0 & 0 & 0 & 0 & $-1 / 2$ \\
\hline
\end{tabular}

Other useful operators are:

$$
\begin{aligned}
& I_{+}=\left(\begin{array}{lll}
0 & 0 & 0 \\
1 & 0 & 0 \\
0 & 1 & 0
\end{array}\right) \quad I_{-}=\left(\begin{array}{lll}
0 & 1 & 0 \\
0 & 0 & 1 \\
0 & 0 & 0
\end{array}\right) \quad I_{z}=\left(\begin{array}{ccc}
-1 & 0 & 0 \\
0 & 0 & 0 \\
0 & 0 & 1
\end{array}\right) \\
& I_{x}=\frac{1}{2}\left(I_{+}+I_{-}\right)=I_{x}^{-1,0}+l_{x}^{0,1}=\left(\begin{array}{ccc}
0 & 1 / 2 & 0 \\
1 / 2 & 0 & 1 / 2 \\
0 & 1 / 2 & 0
\end{array}\right) \\
& I_{y}=\frac{i}{2}\left(I_{-}-I_{+}\right)=I_{y}^{-1,0}+l_{y}^{0,1}=\left(\begin{array}{ccc}
0 & -i / 2 & 0 \\
i / 2 & 0 & -i / 2 \\
0 & i / 2 & 0
\end{array}\right) \\
& Q=3 I_{z}^{2}-2=\left(\begin{array}{ccc}
1 & 0 & 0 \\
0 & -2 & 0 \\
0 & 0 & 1
\end{array}\right)
\end{aligned}
$$

We note that these 9 operators (Table 3.1) are a straightforward generalization of Pauli spin matrices, i.e., matrix representations of $I_{x}, I_{y}$ and $I_{z}$, 
for $I=1 / 2$. Moreover, these matrices transform under rotations in the same way as the classical magnetization vectors in Larmor's theorem.

A convenient way to trace orthogonalize $\left(\mathrm{TrO}_{i} \mathrm{O}_{j}=\delta_{i j}\right)$ the basis operators introduced above, is to replace the $z$ components, $\mathrm{I}_{z}^{-1,0}, \mathrm{I}_{z}^{0,1}$ and $\mathrm{I}_{z}^{-1,1}$, with $\mathrm{I}_{z}, \mathrm{H}_{\mathrm{a}}$ and 1 (respectively, $\mathrm{I}_{z}^{-1,0}+\mathrm{l}_{z}^{0,1}+\mathrm{I}_{z}^{-1,1}, \mathrm{Q}$ related to the quadrupolar Hamiltonian and the identity matrix). The expectation value of $I_{z}$ corresponds to the net $z$ magnetization or Zeeman order and the expectation value of $H_{q}$ is similarly referred to as quadrupolar order. With the 6 single transition coherences, $i_{k}^{i, j}$ $(k=x, y)$, these operators span the 9-dimensional space of $I=1$. Since $\rho=\sum_{i} a_{i} O_{i}$, multiplication of both sides by $O_{j}$ gives: $O_{j} \rho=\sum_{i} a_{i} O_{j} O_{i}$. Then, $\operatorname{Tr}\left(\mathrm{O}_{j} \rho\right)=\sum_{i} a_{i} \operatorname{Tr}\left(O_{j} O_{i}\right)$, and orthogonality $\left(\operatorname{TrO}_{i} \mathrm{O}_{j}=\delta_{i j}\right)$ confirms that $\left\langle\mathrm{O}_{i}\right\rangle=a_{i}$, i.e. the expansion coefficient $a_{i}$ is the expectation value $<O_{i}>$. For example, equilibration in a magnetic field generates population difference, but no net transverse magnetization, i.e. $\rho_{\mathrm{eq}}=1+\mathrm{cl}_{\mathrm{z}}$ where $\mathrm{c}$ is the Curie law constant.

\section{Density operator manipulations: RF pulses and quadrupolar coupling}

Evolution of the density matrix has the well-known form of a matrix transformation, $\mathrm{R}^{\mathrm{t}} \rho \mathrm{R}$, where " $\mathrm{t}$ " notes Hermitian conjugate and $\mathrm{R}$ is a propagator. Application of an RF pulse, transforms the density operator by $R=\exp \left(i \omega_{1} l_{k} t\right),(k=x, y)$. This is the form of a rotation, i.e. an RF pulse acts as a rotation about the "k-axis" through an angle $\theta=\omega_{1} t$. Consequently, application of 
a $90^{\circ}$ pulse $\left(\theta=\omega_{1} t\right)$ with phase $\varphi$ to the equilibrium density operator can be summarized by the following

$$
\mathrm{I}_{z} \stackrel{90_{0}}{\longrightarrow} 1 / \sqrt{2}\left(l_{y}^{-1,0}+l_{y}^{0,1}\right) \text {. }
$$

The identity operator and the Curie constant have been dropped since they are not affected by the unitary transformations of quantum mechanics. Simply stated, the $90^{\circ}$ pulse converts a population difference into transverse single quantum coherences of both transitions. If the $90^{\circ}$ pulse is followed by an evolution period in which the spins evolve under chemical shielding alone, i.e., as in solution NMR, then the density operator is subject to the transformation

$$
R=\exp \left(i \Delta \omega_{o s} l_{z} t\right),
$$

and we obtain

$$
1 / \sqrt{2}\left(l_{y}^{-1,0}+l_{y}^{0,1}\right) \stackrel{H_{o s}, t}{\longrightarrow} 1 / \sqrt{2}\left(l_{y}^{-1,0}+l_{y}^{0,1}\right) \cos \left(\Delta \omega_{o s} t\right)-1 / \sqrt{2}\left(l_{x}^{-1,0}+l_{x}^{0,1}\right) \sin \left(\Delta \omega_{o s} t\right)
$$

In geometrical terms, both $1 Q$ coherences rotate about $z$-axis at the same frequency, $\Delta \omega_{o s}$, so they are detected as a single line. Alternatively, in a solid, evolution after the pulse is dominated by the quadrupolar coupling. The transformed density operator is easily calculated by first rewriting $H_{q}$ using single transition operators:

$$
\begin{gathered}
H_{q}=\omega_{q}\left(l_{z}^{-1,0}-l_{z}^{0,1}\right), \\
1 / \sqrt{2}\left(l_{y}^{-1,0}+l_{y}^{0,1}\right) \stackrel{H_{q}, t}{\longrightarrow} 1 / \sqrt{2}\left(l_{y}^{-1,0}+l_{y}^{0,1}\right) \cos \left(\omega_{q} t\right)+1 / \sqrt{2}\left(l_{x}^{-1,0}+l_{x}^{0,1}\right) \sin \left(\omega_{q} t\right) .
\end{gathered}
$$


The two coherences counter rotate at the same frequency, $\omega_{q}$, but with different sense and are observed in the spectrum as a doublet with lines at $\pm \omega_{\mathrm{q}}$. The solid and solution results differ due to the quadratic dependence of $\mathrm{H}_{\mathrm{q}}$ on the angular momentum operator, $\mathrm{I}_{\mathrm{z}}$.

\section{MQ coherence preparation, evolution and detection}

Recall that the goal is to detect the signal from transiently ordered water molecules and suppress the background signal from bulk water, which is, potentially in large excess. The non-zero quadrupolar coupling is the essential feature of the ordered water molecules that can be used for filtration of their signal from the bulk water signal. To implement this, consider the following twopulse train in Figure 3.1 originally described by Ernst and Wokaun [116].

$$
90 x-T-90 x
$$

Figure 3.1. "Pulse sequence" for the preparation of MQ coherence.

For simplicity, a small frequency offset is neglected during the short period, $T=\pi / 2 \omega_{Q}$, and the transformations are:

$$
\mathrm{I}_{z} \stackrel{90_{0}}{\longrightarrow} 1 / \sqrt{2}\left(l_{y}^{-1,0}+l_{y}^{0,1}\right) \stackrel{H_{q}, T=\pi / 2 \omega_{q}}{\longrightarrow} 1 / \sqrt{2}\left(l_{x}^{-1,0}-l_{x}^{0,1}\right) \stackrel{90_{0}}{\longrightarrow}-l_{y}^{-1,1} .
$$

The net effect is that $I_{z}$ is completely converted into double quantum (2Q) coherence. In practice, this sequence is a general method to prepare $2 \mathrm{Q}$ coherence, i.e. it is analogous to a $90^{\circ}$ pulse for the preparation of $1 Q$ coherence. Note, that if $\omega_{q}=0$, no $2 Q$ coherence is created. 


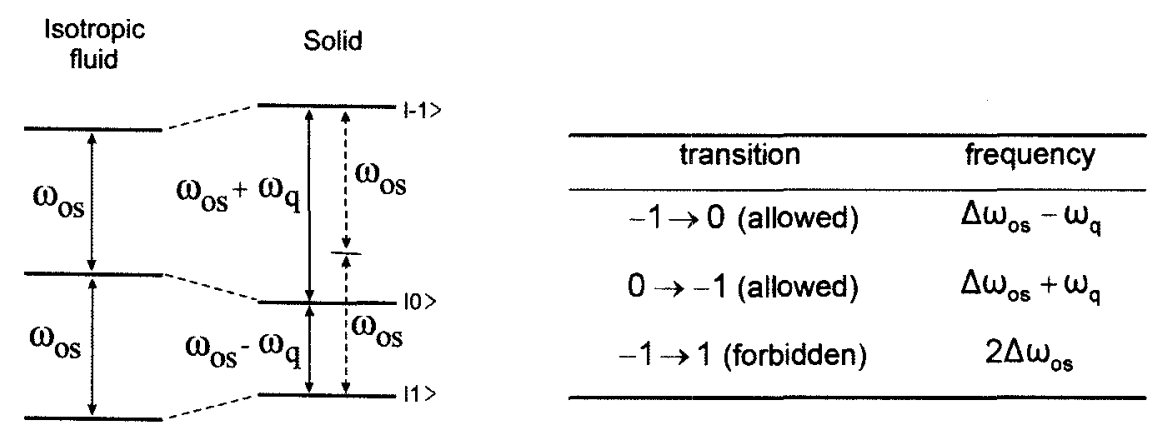

Figure 3.2. Energy level scheme for nuclei with $I=1$.

Another unique feature of $M Q$ coherence is their dependence on the offset frequency. The observed offset for an $n^{\text {th }}$-order coherence is $n \Delta \omega_{o s}$. Consider $2 \mathrm{Q}$ coherence example. The energy level scheme in Figure 3.2 shows two familiar cases. In isotropic fluids, the quadrupolar coupling is zero and the allowed transitions appear as a single line at the chemical shift frequency, while in a solid a doublet centered at $\Delta \omega_{o s}$ with splitting $2 \omega_{q}$ is observed. As anticipated, $2 Q$ coherence evolves at twice the offset frequency $2 \Delta \omega_{o s}$ and is independent of the quadrupolar coupling. Note, no independence on $\omega_{q}$ drove the initial interest in the detection of $M Q$ transitions, since the corresponding spectral lines have no quadrupolar broadening and thus increases resolution.

The signal induced in the NMR coil is proportional to $I_{y}=I_{y}^{-1,0}+l_{y}^{0,1}$ and $I_{x}=I_{x}^{-1,0}+I_{x}^{0,1}$, which are orthogonal to $I_{k}^{-1,1},(k=x, y)$. Thus, $2 Q$ coherence cannot be directly detected. To circumvent this problem, an indirect dimension, the evolution dimension, is introduced during which the $2 Q$ coherence evolves as a function of time. Then $2 Q$ coherence is transferred to single quantum coherence that is then detected as a function of $t_{2}$ yielding a $2 D$ array of free induction 
decays (FID's), $S\left(t_{1}, t_{2}\right)$. It is important to understand, that coherence transfer in itself, does not provide the desired $2 \mathrm{Q}$ selectivity, and it is essential for the spins to undergo a coherence specific manipulation, such as the phase shift of the transmitter and/or the offset dependence, to give coherence specific filtration. In other words, the coherences (1Q coherence or transverse magnetization) are subjected to their characteristic response to shifts of the transmitter phase and/or offset frequency in such a way that $1 \mathrm{Q}$ coherence is either filtered out or, in a $2 \mathrm{D}$ experiment, separated in frequency from MQ coherences.

The evolution of $2 Q$ coherence under $H_{q}$ and $H_{o s}$ is given by

$$
--_{y}^{-1,1} \stackrel{H_{q}+H_{o s}, t_{1}}{\longrightarrow}--_{y}^{-1,1} \cos \left(2 \Delta \omega_{o s} t_{1}\right) .
$$

Conversion of $2 \mathrm{Q}$ coherence to single quantum coherence is affected by a $90^{\circ}$ pulse:

$$
-\mathrm{l}_{y}^{-1,1} \cos \left(2 \Delta \omega_{o s} t_{1}\right) \stackrel{90_{0}}{\longrightarrow}-\frac{1}{4} \cos \left(2 \Delta \omega_{o s} t_{1}\right)\left(l_{y}^{-1,0}+l_{y}^{0,1}\right)
$$

Double Fourier transformation of $S\left(t_{1}, t_{2}\right)$ yields a two-dimensional spectrum with an indirect frequency axis, $F_{1}$, and a direct or detection axis, $F_{2}$. The $2 \mathrm{Q}$ transition is observed as a signal at twice the offset frequency in the indirect dimension, $F_{1}$, and only ordered water deuterons with non-zero quadrupolar coupling give signals at $F_{1}=2 \Delta \omega_{O S}$. 


\subsection{Pulse sequence and phase cycle development}

\section{Pulse sequence}

The formalism described above is readily implemented by numerical methods for simulating any pulse sequence. We simulate pulse sequences and phase cycles using a scripted language, Octave [117]. As a starting point we have used the pulse sequence shown in Figure 3.3 [84-87, 93-96].

$$
\begin{aligned}
& 90_{\alpha}-\frac{t_{1}}{2}-180_{\alpha+90}-\frac{t_{1}}{2}-90_{\alpha}-t_{2}-90_{Y}-\text { FID } \\
& \left|\quad \operatorname{prepare}\left(t_{1}\right) \quad-\right| \text { evolve }\left(t_{2}\right) \mid \text { convert/detect }\left(t_{3}\right) \mid
\end{aligned}
$$

Figure 3.3. Simple pulse sequence for MQ NMR.

To summarize, note that after the $1^{\text {st }} 90^{\circ}$ pulse, transverse magnetization from the two 1Q coherences evolve under the quadrupolar coupling and fan out at the angular frequencies $\pm \omega_{\mathrm{q}}$. The $180^{\circ}$ pulse refocuses the offset interaction so that it has no net effect during the preparation period, and the accumulated phase difference of the $1 Q$ coherences is $2 \omega_{q} t_{1}$. With $\omega_{q} t_{1}=45^{\circ}\left(t_{1}=1 /\left(4 v_{q}\right)\right)$, the $1 Q$ coherences are "antiparallel" and the state is pure $1 \mathrm{Q}$ anti-phase coherence, $I_{x}^{-1,0}-I_{x}^{0,1}$. The $2^{\text {nd }} 90^{\circ}$ pulse converts anti-phase coherence into $2 Q$ coherence. The $2 \mathrm{Q}$ coherence (unobservable) precesses at twice the offset frequency during the evolution period. Since coherent evolution of spins is unitary, the final $90^{\circ}$ pulse converts $2 Q$ coherence back to observable anti-phase $1 Q$ coherence, and a doublet with signals of opposite phase are detected. An obvious problem with this sequence is that if the quadrupolar coupling is comparable to the linewidth, 
the two $1 \mathrm{Q}$ coherences that are observed have opposite phase and signal cancellation occurs.

To overcome this, we have implemented the usual density operator formalism to account for both coherent evolution and relaxation of the spin system, and designed the pulse sequence shown in Figure 3.4.

$$
\begin{aligned}
& 90_{\alpha}-\frac{t_{1}}{2}-180_{\alpha+90}-\frac{t_{1}}{2}-90_{\alpha}-\frac{T-t_{2}}{2}-180_{\beta}-\frac{T+t_{2}}{2}-t_{2}-90_{V}-\frac{t_{1}}{2}-180_{Y+90}-\frac{t_{1}}{2}-90_{Y}-T^{\prime}-900-\text { FID } \\
& +\quad \text { prepare }\left(t_{1}\right) \rightarrow \text { evolve }\left(T, t_{2}\right)+\quad+\quad \text { convert }\left(t_{1}\right)=\left|\operatorname{detect}\left(T^{\prime}, t_{3}\right)\right|
\end{aligned}
$$

Figure 3.4. Advanced pulse sequence for the detection of MQ coherence.

\section{$2 Q$ preparation}

The preparation period is equivalent to that described above and the overall transformation in this period is

$$
\mathrm{I}_{z} \stackrel{90_{a}-\frac{\mathrm{t}_{1}}{2}-180_{a+80}-\frac{\mathrm{t}_{1}}{2}-90_{a}}{\longrightarrow}-\cos \left(\omega_{\mathrm{q}} \mathrm{t}_{1}\right) \mathrm{I}_{z}+2 \sin \left(\omega_{\mathrm{q}} \mathrm{t}_{1}\right)\left[\mathrm{l}_{\mathrm{y}}^{-1,1} \cos (2 \alpha)-\mathrm{I}_{\mathrm{x}}^{-1,1} \sin (2 \alpha)\right] .
$$

For example, advancing the transmitter phase a from $0^{\circ}$ to $90^{\circ}$, leaves the polarization unchanged and inverts $2 Q$ coherence. In general, if rotating the transmitter phase by $\alpha$ rotates the $1 Q$ by $\alpha$, then the $2 Q$ coherence is rotated in the same sense by $2 \alpha$.

\section{Constant time evolution}

Typically, $1 \mathrm{Q}$ signals are eliminated in $2 \mathrm{Q}$-filtered experiments by phase cycling, i.e., $2 Q$ filtration. However, in cases where the $1 Q$ and $2 Q$ signals overlap and the former is potentially much larger, the demands on filtration are very high, so it is useful to also separate signals by the order of their coherence, 
i.e., their different dependence on the offset frequency. We use a constant time evolution period so that relaxation effects during the evolution period are independent of the evolution time, $t_{1}$, and depend only on the length of the evolution period, $\mathbf{T}$, which should be kept to a minimum for purposes of good signal to noise. Finally, given an efficient probe, $v_{o s}$ can be made sufficiently large so that $T$ is short compared to $T_{2}^{2 Q}$ (transverse relaxation time of $2 Q$ signal). In this way, there is negligible signal loss due to relaxation during evolution.

Since $I_{z}$ commutes with $H_{q}$ and $H_{o s}$, it is stationary and only $2 Q$ coherence evolves during the evolution period. If $t_{1}=0$, it is readily shown that $2 \mathrm{Q}$ coherence is refocused. Thus, aside from a phase shift and relaxation, the density operator is unchanged in the evolution period for arbitrary $\mathrm{T}$. Straightforward calculation shows that for non-zero $t_{1}, 2 Q$ coherence rotates clockwise in the rotating frame at frequency $2 \omega_{o s}$ as follows:

$$
\rho\left(\alpha, t_{1}, t_{2}\right)=\left\{\begin{array}{c}
l_{z} \cos \left(\omega_{q} t_{1}\right)-2 \cos (2 \alpha) \cos (4 \beta) \sin \left(\omega_{q} t_{1}\right) \\
\times\left[l_{y}^{-1,1} \cos \left(2 \omega_{o s} t_{2}\right)+l_{x}^{-1,1} \sin \left(2 \omega_{o s} t_{2}\right)\right], \alpha=0^{\circ}, 90^{\circ} \ldots \\
l_{z} \cos \left(\omega_{q} t_{1}\right)-2 \cos (2(\alpha-45)) \cos (4 \beta) \sin \left(\omega_{q} t_{1}\right) \\
\times\left[l_{x}^{-1,1} \cos \left(2 \omega_{o s} t_{2}\right)-l_{y}^{-1,1} \sin \left(2 \omega_{o s} t_{2}\right)\right], \alpha=45^{\circ}, 135^{\circ} \ldots
\end{array}\right\},
$$

i.e., the density operator contains three terms: $I_{z}, I_{y}^{1,1}$ and $I_{x}^{-1,1}$. 


\section{Conversion}

The transformations of quantum mechanics are unitary so the conversion of $2 \mathrm{Q}$ coherence to observable $1 \mathrm{Q}$ coherence is analogous to the preparation period. With $Y=0^{\circ}$ and $t_{1}=1 / 4 v_{q}$ the transformations are

$$
\begin{aligned}
& \mathrm{I}_{\mathrm{z}} \stackrel{90_{0}}{\longrightarrow} \mathrm{I}_{\mathrm{y}} \stackrel{\frac{\mathrm{t}_{1}}{2}-(180)_{80}-\frac{\mathrm{t}_{1}}{2}}{\longrightarrow} \sqrt{2}\left(\mathrm{I}_{\mathrm{x}}^{-1,0}-\mathrm{I}_{\mathrm{x}}^{0,1}\right) \\
& \mathrm{I}_{x}^{-1,1} \stackrel{90_{0}}{\longrightarrow} 1 / 2\left(\mathrm{l}_{x}^{-1,1}-1 / 6 \mathrm{Q}\right) \stackrel{\frac{\mathrm{t}_{1}}{2}-(180)_{0}-\frac{\mathrm{t}_{1}}{2}}{\longrightarrow} 1 / 2\left(\mathrm{l}_{x}^{-1,1}-1 / 6 \mathrm{Q}\right) \\
& I_{y}^{-1,1} \stackrel{90_{0}}{\longrightarrow}-1 / \sqrt{2}\left(I_{x}^{-1,1}-I_{x}^{0,1}\right)-\stackrel{\frac{t_{1}}{2}-(180)_{90}-\frac{t_{1}}{2}}{\longrightarrow} I_{y} .
\end{aligned}
$$

\section{Alignment and purge}

The $I_{z}$ term in the evolution period evolves to an observable signal, which is unwanted for $2 Q$ filtration and eliminated by a suitable phase cycle. Whereas $\mathrm{I}_{\mathrm{y}}^{-1,1}$ is completely converted into an observable signal by pulse phases that are integral multiples of $90^{\circ}, \mathrm{l}_{\mathrm{x}}^{-1,1}$ is similarly converted only if the phase is an oddintegral multiple of $45^{\circ}$. Consequently, conversion of $2 \mathrm{Q}$ coherence selects either $\mathrm{I}_{y}^{-1,1}$ or $\mathrm{l}_{x}^{-1,1}$. In other words, the signal originating from $2 \mathrm{Q}$ coherence is amplitude rather than phase modulated in $t_{2}$.

Observable $1 \mathrm{Q}$ coherences created in this way are in anti-phase and are aligned by the $180^{\circ}$ pulse with delay $t_{1}=1 / 4 v_{q}$. Unoriented samples, for example, have a distribution of couplings so the anti-phase coherences cannot, 
in general, be "refocused", i.e., realigned independent of $v_{q}$. For arbitrary $t_{1}$ and $Y=0^{\circ}, 90^{\circ}, 180^{\circ}$ and $270^{\circ}$ the result is

$$
r_{y}^{-1,1} \stackrel{90_{y}-\frac{t_{1}}{2}-180_{y+90}-\frac{t_{1}}{2}}{\longrightarrow}\left\{\begin{array}{l} 
\pm 1 / 2\left(l_{x}^{0,1}-l_{x}^{-1,0}\right) \cos \omega_{q} t_{1} \pm l_{y} \sin \omega_{q} t_{1}, \quad \text { for } y=0^{\circ} \text { or } 180^{\circ} \\
\pm 1 / 2\left(l_{y}^{-1,0}-l_{y}^{0,1}\right) \cos \omega_{q} t_{1} \pm l_{x} \sin \omega_{q} t_{1}, \quad \text { for } y=90^{\circ} \text { or } 270^{\circ}
\end{array}\right\} \text {. }
$$

From the above, it is apparent that phases of undesired anti-phase and desired in-phase coherence shift in the same way with the transmitter phase. Therefore a phase cycle cannot be used to cancel the anti-phase components while preserving the in-phase components. An alternative, used here, is a purge: two $90^{\circ}$ pulses separated by a delay, $T^{\prime}$, such that $T_{2}^{2 Q} \ll T^{\prime} \ll T_{1}\left(T_{1}\right.$ is longitudinal relaxation time). The initial pulse converts the anti-phase component into rapidly decaying $2 Q$ coherence and stores the in-phase component as long lasting $\mathrm{I}_{\mathrm{z}}$. Following the delay, $\mathrm{I}_{\mathbf{z}}$ is reconverted to the in-phase signal. Effectively, the purge period removes the anti-phase component from the signal.

The consequences of eliminating the alignment of $1 Q$ coherences and not purging are shown in Figure 3.5, for two cases, when the quadrupolar doublet is larger than or comparable to the $1 \mathrm{Q}$ linewidth. The latter is of particular importance, for example, when a solvent interacts with a surface and is only weakly ordered. In this case, there is severe signal loss from cancellation of the two coherences of the opposite phase. Superficially, the absorption and dispersion signals appear interchanged and the latter is typically reported with the characteristic feature that the wings of the signal drop below the baseline. With refocusing, there is no signal cancellation and the absorption mode signal is 
observed with greater sensitivity. With the purge, peak intensities are independent of the linewidth and proportional to $\sin ^{2} \omega_{q} t_{1}$.

A recent study of elastin [112-113] by $2 Q$ NMR reports dispersion signal which hampers quantitative analysis. The pulse sequence designed here successfully circumvents these problems due to addition of alignment and purging and allows observation of an absorption mode signals. 

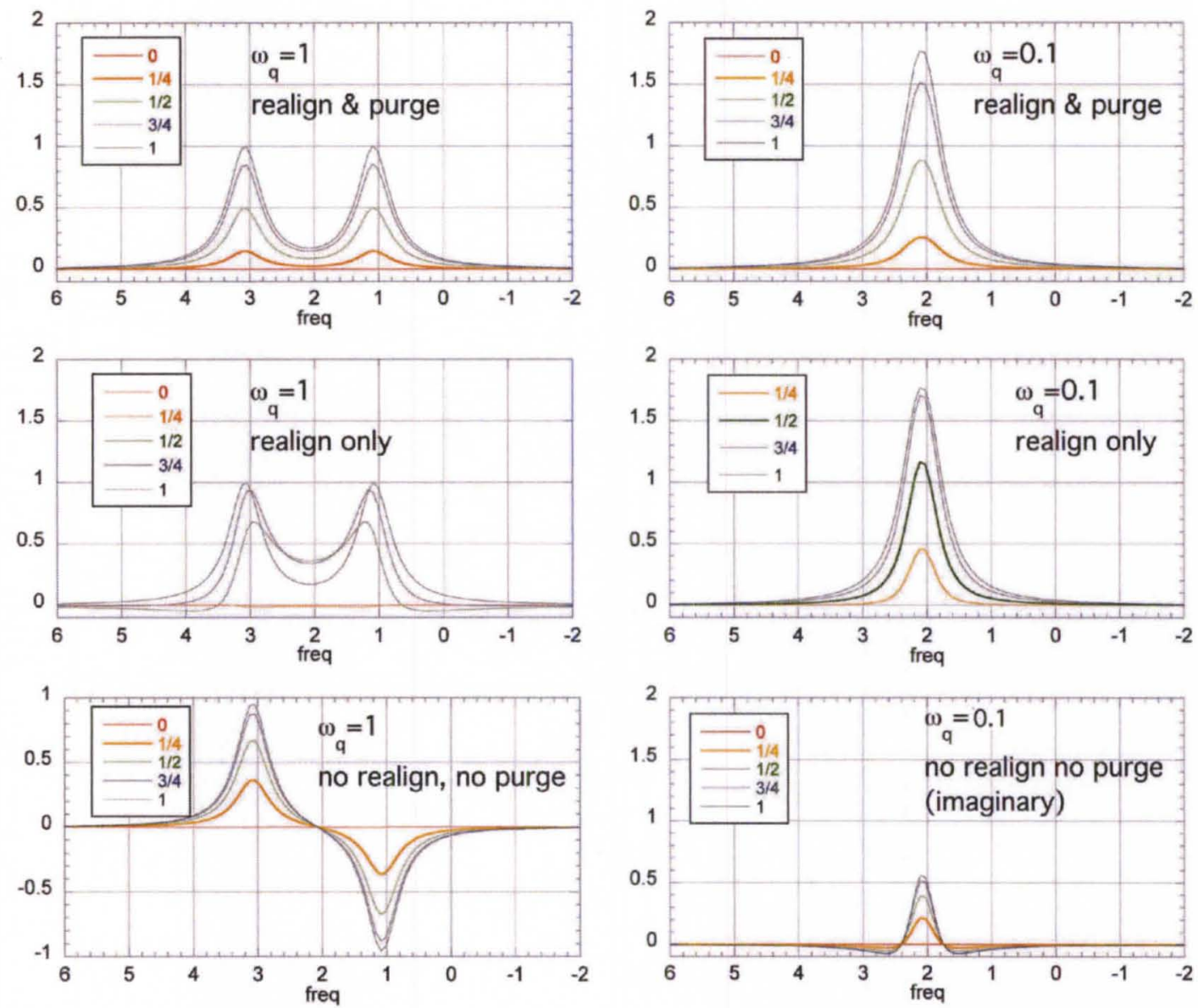

Figure 3.5. Simulated $2 Q$ spectra as a function of the preparation/conversion time, $t_{1}$ (in boxes as a fraction of $1 / 4 v_{q}$ ). Spectra on the left have resolved quadrupolar splittings and on the right the splitting, while non-zero, is obscured by the linewidth. In the top row, the complete sequence described herein is used and signal intensities are $\prec \sin ^{2} \omega_{\mathrm{q}} t_{1}$. In the middle row, purge is eliminated and the signal intensity in the weakly coupled case depends also on the linewidth. In the bottom row, alignment and purge are eliminated. Here, the $1 \mathrm{Q}$ coherences are observed in anti-phase with nearly complete cancellation of the absorption mode signal in the weak coupling case. The more easily recognized dispersion signal is shown. 


\section{Phase cycle}

It is important to note, that the pulse sequence alone does not provide the necessary elimination of the $1 \mathrm{Q}$ signal, and the role of the phase cycle should not be underestimated. The phase cycle is responsible for the elimination of unwanted coherences and consequently determines what is detected during $t_{3}$.

The signal arising from the $I_{z}$ component of the density operator at the end of the preparation period is removed by cycling the preparation phase. For example, shifting $\alpha$ by $90^{\circ}$ inverts the $2 Q$ coherence and leaves the signal originating from $\mathrm{I}_{2}$ unchanged, so subtracting the resulting signals removes the later. The signal arising from the spins with zero quadrupolar coupling is also eliminated by the phase cycle.

Table 3.2 summarizes a 16-step phase cycle that, in principle, suffices to accomplish the goal of complete $1 \mathrm{Q}$ cancellation. In practice, to cancel also artifacts originating from misset pulse widths and hardware, we use a 64-step phase cycle. The phase of the refocusing pulse in the evolution period is cycled $45^{\circ}, 135^{\circ}, 225^{\circ}$ and $315^{\circ}$, for each of the 16 steps in Table 3.2. Overall, the sequence has accumulated 64 steps. Consequently, the number of transients in the experiment should be a multiple of 64 in order to cycle through all the phases equally. 
Table 3.2. Phase cycle to cancel $1 Q$ signal.

\begin{tabular}{|c|c|c|c|c|c|c|c|c|c|c|c|}
\hline step & $\alpha$ & $\beta$ & $\gamma$ & start & $\begin{array}{l}\text { prep, } \\
2 \mathrm{Q}\end{array}$ & $\begin{array}{l}\text { evol, } \\
2 Q^{*}\end{array}$ & $\begin{array}{l}\text { conv, } \\
2 \mathrm{Q}\end{array}$ & $\begin{array}{l}\text { prep, } \\
10\end{array}$ & $\begin{array}{l}\text { evol, } \\
1 Q\end{array}$ & $\begin{array}{l}\text { conv, } \\
1 \mathrm{Q}\end{array}$ & revr \\
\hline 1 & 0 & 45 & 0 & $I_{z}$ & $+a l_{y}^{-1,1}$ & $+\left.a^{\prime}\right|_{y} ^{-1,1}$ & $-a^{\prime \prime} I_{y}$ & $-b l_{z}$ & $+b I_{z}$ & $-\left.2 b\right|_{y}$ & 0 \\
\hline 2 & 0 & 45 & 90 & $"$ & $+a I_{y}^{-1,1}$ & $+\left.a^{\prime}\right|_{y} ^{-1,1}$ & $-\left.a^{\prime \prime}\right|_{y}$ & $-b l_{z}$ & $+b_{z}$ & $+\left.2 b\right|_{x}$ & 90 \\
\hline 3 & 0 & 45 & 180 & $"$ & $+a_{y}^{-1,1}$ & $+a^{\prime} I_{y}^{-1,1}$ & $+a^{\prime \prime} I_{y}$ & $-b l_{z}$ & $+\mathrm{bl}_{z}$ & $+\left.2 b\right|_{y}$ & 180 \\
\hline 4 & 0 & 45 & 270 & “ & $+\left.a\right|_{y} ^{-1,1}$ & $+a^{\prime} \mathrm{I}_{\mathrm{y}}^{-1,1}$ & $+a^{\prime \prime} I_{y}$ & $-b I_{z}$ & $+b I_{z}$ & $-\left.2 b\right|_{x}$ & 270 \\
\hline 5 & 90 & 45 & 0 & " & $-\left.a\right|_{y} ^{-1,1}$ & $-\left.a\right|_{y} ^{-1,1}$ & $+a^{n} I_{y}$ & $-b I_{z}$ & $+b l_{z}$ & $-\left.2 b\right|_{y}$ & 180 \\
\hline 6 & 90 & 45 & 90 & $"$ & $-\left.a\right|_{y} ^{-1,1}$ & $-\left.a\right|_{y} ^{-1,1}$ & $+a^{\prime \prime} I_{y}$ & $-b I_{z}$ & $+\mathrm{bl}_{z}$ & $+\left.2 b\right|_{x}$ & 270 \\
\hline 7 & 90 & 45 & 180 & $"$ & $-\left.a\right|_{y} ^{-1,1}$ & $-\mathrm{al}_{\mathrm{y}}^{-1,1}$ & $-\left.a^{\prime \prime}\right|_{y}$ & $-b l_{z}$ & $+b_{z}$ & $+\left.2 b\right|_{y}$ & 0 \\
\hline 8 & 90 & 45 & 270 & $"$ & $-\left.a\right|_{y} ^{-1,1}$ & $-\left.a\right|_{y} ^{-1,1}$ & $-a^{\prime \prime} I_{y}$ & $-b I_{z}$ & $+b I_{z}$ & $-\left.2 b\right|_{x}$ & 90 \\
\hline 9 & 180 & 45 & 0 & $"$ & $+a l_{y}^{-1,1}$ & $+a^{\prime} I_{y}^{-1,1}$ & $-a^{\prime \prime} I_{y}$ & $-b I_{z}$ & $+b_{z}$ & $-\left.2 b\right|_{y}$ & 0 \\
\hline 10 & 180 & 45 & 90 & $"$ & $+a l_{y}^{-1,1}$ & $+a^{\prime} I_{y}^{-1,1}$ & $-a^{\prime \prime} I_{y}$ & $-b I_{z}$ & $+b l_{z}$ & $+\left.2 b\right|_{x}$ & 90 \\
\hline 11 & 180 & 45 & 180 & $"$ & $+\left.a\right|_{y} ^{-1,1}$ & $+a^{\prime} I_{y}^{-1,1}$ & $+a^{\prime \prime} I_{y}$ & $-b I_{z}$ & $+b I_{z}$ & $+\left.2 b\right|_{y}$ & 180 \\
\hline 12 & 180 & 45 & 270 & $"$ & $+a l_{y}^{-1,1}$ & $+a^{\prime} I_{y}^{-1,1}$ & $+a^{\prime \prime} I_{y}$ & $-b I_{z}$ & $+b \mathrm{I}_{z}$ & $-\left.2 b\right|_{x}$ & 270 \\
\hline 13 & 270 & 45 & 0 & $"$ & $-\left.a\right|_{y} ^{-1,1}$ & $-a l_{y}^{-1,1}$ & $+\left.a^{\prime \prime}\right|_{y}$ & $-b I_{z}$ & $+b l_{z}$ & $-\left.2 b\right|_{y}$ & 180 \\
\hline 14 & 270 & 45 & 90 & $"$ & $-a l_{y}^{-1,1}$ & $-a l_{y}^{-1,1}$ & $+a^{\prime \prime} I_{y}$ & $-b I_{z}$ & $+\mathrm{bl}_{\mathrm{z}}$ & $+\left.2 b\right|_{x}$ & 270 \\
\hline 15 & 270 & 45 & 180 & $"$ & $-\mathrm{al}_{y}^{-1,1}$ & $-\mathrm{al}_{\mathrm{y}}^{-1,1}$ & $-a^{\prime \prime} I_{y}$ & $-\mathrm{bl}_{z}$ & $+\left.b\right|_{z}$ & $+\left.2 b\right|_{y}$ & 0 \\
\hline 16 & 270 & 45 & 270 & $"$ & $-\left.a\right|_{y} ^{-1,1}$ & $-a l_{y}^{-1,1}$ & $-a^{\prime \prime} I_{y}$ & $-b I_{z}$ & $+b l_{z}$ & $-\left.2 b\right|_{x}$ & 90 \\
\hline${ }^{+}$Sum & & & & & & & $-\left.16 a^{n}\right|_{y}$ & & & 0 & \\
\hline
\end{tabular}

${ }^{*}$ The term containing $\mathrm{I}_{\mathrm{x}}^{-1,1}$ is neglected since it is not converted into an observable.

+ Signals are rotated according to the receiver phase before summing. 


\subsection{Types of experiments}

Depending on the experimental goal, it is possible to construct different types of experiments by slight variations of the pulse sequence on Figure 3.4.

\section{Preparation curve}

To determine the quadrupolar coupling, the evolution time $\mathrm{T}$ is fixed and $t_{1}$ values for the preparation/conversion times are incremented. Fourier transform of $S\left(t_{1}, t_{3}\right)$ with respect to $t_{3}$ yields Figure 3.6 , obtained from an elastin sample.

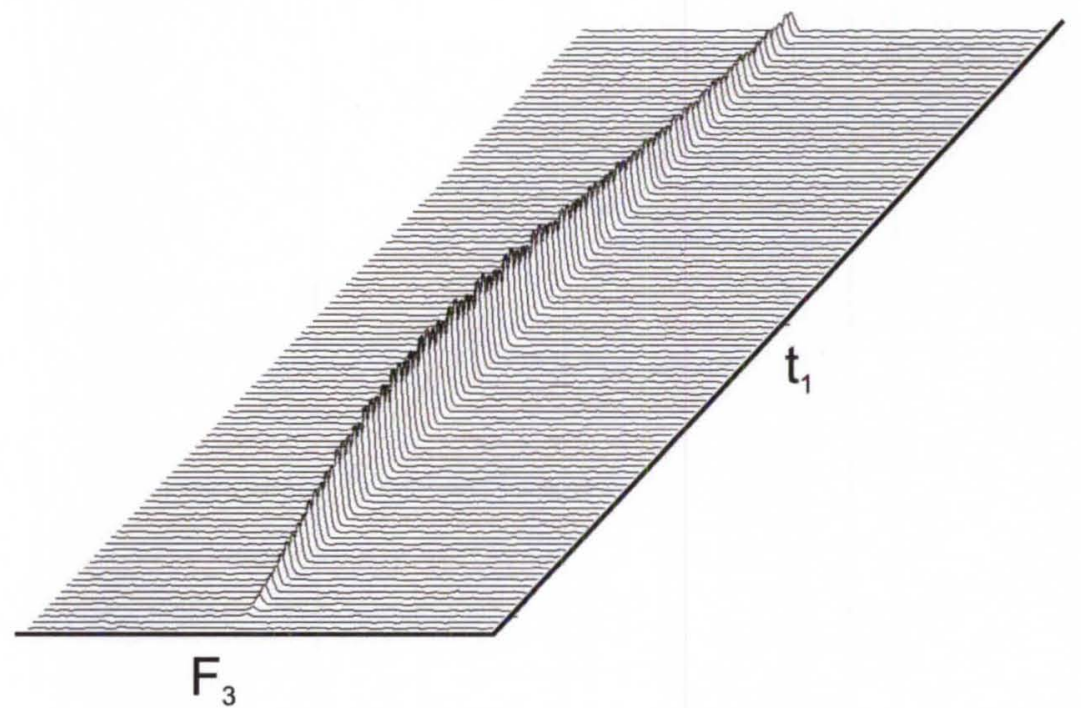

Figure 3.6. Stack plot of preparation spectra, obtained from elastin sample. Only 100 of spectra are shown (490 were taken). The peak in each spectrum corresponds to the point of the preparation curve.

The ridge plotted as a function of $t_{1}$ is hereafter referred to as a preparation curve. In practice, small oscillations of the ridge plot at the offset frequency are observed. This results from small errors in $90^{\circ}$ pulse widths and transmitter phases. These have been removed by the simple expedient of using an offset frequency that is large compared to the rate at which $2 Q$ coherence 
evolves, i.e. $v_{O S} \gg v_{q}$. After Fourier transformation of the preparation curve, offset signals are removed by zeroing the wings of the spectrum. Inverse Fourier transformation returns the undistorted preparation curve.

\section{Evolution planes}

The constant time evolution period allows separation of coherences by order because of their different dependence on the offset frequency: $1 Q$ coherence precesses at the offset frequency, and $2 Q$ coherence precesses at double the offset frequency. When the preparation time is kept constant and the length of the evolution period varies, we obtain a 2D spectrum composed of the evolution planes (Figure 3.7).

To perform this experiment efficiently, a minimal number, $2^{n}$, of $t_{2}$ increments can be recorded without causing truncation artifacts if the acquisition time, $2^{n} d_{2}\left(d_{2}\right.$ is the dwell time (d) in the indirect dimension) is an integral multiple of the offset period, $1 / v_{\text {OS }}$. In practice, we collect data for two offset periods with 8 points, $d_{2}=1 / 4 v_{O S}$, and apply a complex Fourier transform in $t_{2}$. This gives $8 F_{1}$ slices at integral multiples of the offset frequency, i.e. $F_{1}=-3 v_{O S} \ldots 2 v_{O S} \ldots 4 v_{O S}$ and the amplitude modulated $2 Q$ signal appears at $F_{1}= \pm 2 v_{o s}$ 
(a)



F3 (b)

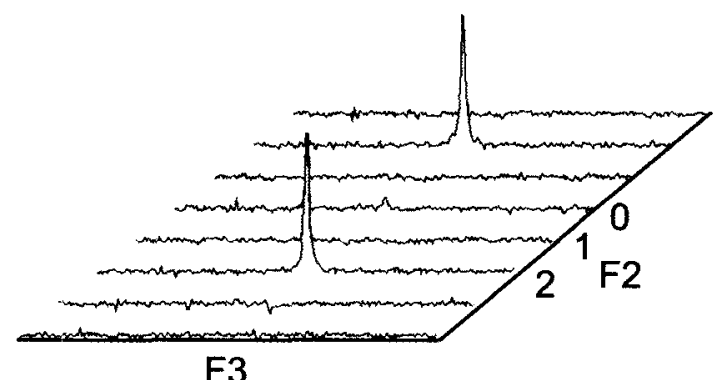

F3

Figure 3.7. Example of evolution plane, obtained from elastin sample using 16-step phase cycle (a) and 64-step phase cycle (b).

In the 2D evolution spectra, Figure 3.7 , the direct dimension is $F_{3}$ (detection) and the $2 Q$ frequencies appear in the indirect dimension, $F_{2}$. Figure 3.7 (a) shows incomplete $1 \mathrm{Q}$ cancellation with a 16 -step phase cycle. This $1 \mathrm{Q}$ "signal" would contribute to the signal, for example, in the preparation experiment, spoiling the result. Efficient 1Q silencing (Figure $3.7(b)$ ) is, however, verified for the 64-step phase cycle discussed above.

3.5. Analysis of experimental data - preparation curve fitting

During the preparation (or conversion sequence) of length $t_{1}$, single quantum coherence evolves from (or to) $I_{z}$ into (or from) $2 Q$ coherence with transfer efficiency proportional to $\sin \omega_{q} t_{1}$. Thus, the overall efficiency of the sequence for the detection of $2 Q$ coherence is $\sin ^{2} \omega_{q} t_{1}$. In addition to coherent evolution, there is relaxation that, in accord with Redfield theory, results in exponential decay, $e^{-t_{1} / T_{2}^{\text {surf }}}$, where $T_{2}^{\text {surf }}$ is the decay time for the $1 Q$ coherences 
that contribute to the $2 Q$ signal. Consequently, the overall transfer efficiency of the sequence for the fixed evolution time is

$$
S_{2 Q}\left(t_{1}\right)=A_{2 Q} \exp \left(-2 t_{1} / T_{2}^{\text {surf }}\right) \sin ^{2}\left(\omega_{q} t_{1}\right)
$$

where $A_{2 Q}$ is a constant proportional to the number of spins with $\omega_{q} \neq 0$.

For the randomly oriented samples, like elastin fibers, the presence of a non-zero quadrupolar coupling necessarily implies a distribution of quadrupolar frequencies, $\omega_{\mathrm{q}}$, since there is a random orientation of the coupling tensor with respect to the magnetic field. The expression for $S_{2 Q}\left(t_{1}\right)$ can be generalized for this by explicitly including the orientation dependence in the quadrupolar frequency, $\omega_{\mathrm{q}}(\theta)$, and integrating over the orientations, $\theta$,

$$
S_{2 Q}\left(t_{1}\right)=A_{2 Q} \exp \left(-2 t_{1} / T_{2}^{\text {surf }}\right) \int \sin ^{2}\left(\omega_{q}(\theta) t_{1}\right) d \theta
$$

or by integrating over quadrupolar frequencies with an appropriate weighting factor, $P\left(\omega_{q}\right)$, that accounts for a randomly oriented sample

$$
S_{2 Q}\left(t_{1}\right)=A_{2 Q} \exp \left(-2 t_{1} / T_{2}^{\text {surf }}\right) \int \sin ^{2}\left(\omega_{q} t_{1}\right) P\left(\omega_{q}\right) d \omega_{q}
$$

To implement either, we assume that the residual coupling is axially symmetric (Figure 3.8 ), so $\omega_{q}(0)=\omega_{q}\left(3 \cos ^{2} \theta-1\right) / 2$. With the substitution, $x=\cos \theta$, the first case is then

$$
S_{2 Q}\left(t_{1}\right)=A_{2 Q} \exp \left(-2 t_{1} / T_{2}^{\text {surf }}\right) \int_{0}^{1} \sin ^{2}\left(\frac{1}{2} \omega_{q}\left(3 x^{2}-1\right) t_{1}\right) d x
$$


The function $\mathrm{P}\left(\omega_{\mathrm{q}}\right)$ has the shape of the usual powder pattern for a single transition, shown in Figure 3.8 and calculated numerically for two different linewidths.



Figure 3.8. Calculated $P\left(\omega_{\mathrm{q}}\right)$ for an axially symmetric residual quadrupolar coupling, $\omega_{\mathrm{q}}=200 \mathrm{~Hz}$ and linewidths of $1 \mathrm{~Hz}$ and $50 \mathrm{~Hz}\left(\mathrm{~T}_{2}^{\text {surf }}=1 / \pi \Delta \mathrm{v}=318 \mathrm{~ms}\right.$ and $6.4 \mathrm{~ms}$, respectively). 
$\mathrm{S}_{2 Q}$ in the case of a single coupling is shown in Figure 3.9 (top), both in the absence of relaxation and with $\mathrm{T}_{2}^{\text {surf }} \approx 6 \mathrm{~ms}$ (linewidth $=50 \mathrm{~Hz}$ ). Simulations of this type have been used before, but agreement with experimental results is only approximate for short times $\left(t_{1}<5 \mathrm{~ms}\right)$ and, for longer times fails since the oscillations are not observed.

Simulations based on numerical integrations of eq. 1 and eq. 2 are shown in Figure 3.9 (bottom). In the absence of relaxation (black solid curve, calculations based on eq. 1 and circles for calculations based on eq. 2), the transfer efficiency builds up at a rate that increases with $\omega_{q}$ and then oscillates. However, unlike the single frequency case (top, no relaxation, black line), a nonzero limit of $1 / 2$ is approached. Calculations based on eq. 1 (red solid curve) and eq. 2 with $1 \mathrm{~Hz}$ broadening in $\mathrm{P}\left(\omega_{\mathrm{q}}\right)$ (red circles) are essentially equivalent. With the addition of relaxation, $\mathrm{T}_{2}^{\text {surf }}=6.36 \mathrm{~ms}$, the transfer efficiency decays rapidly as expected. Matching the linewidth of $P\left(\omega_{q}\right)$ to the decay rate shows a smooth, monotonic decay (solid purple line). 

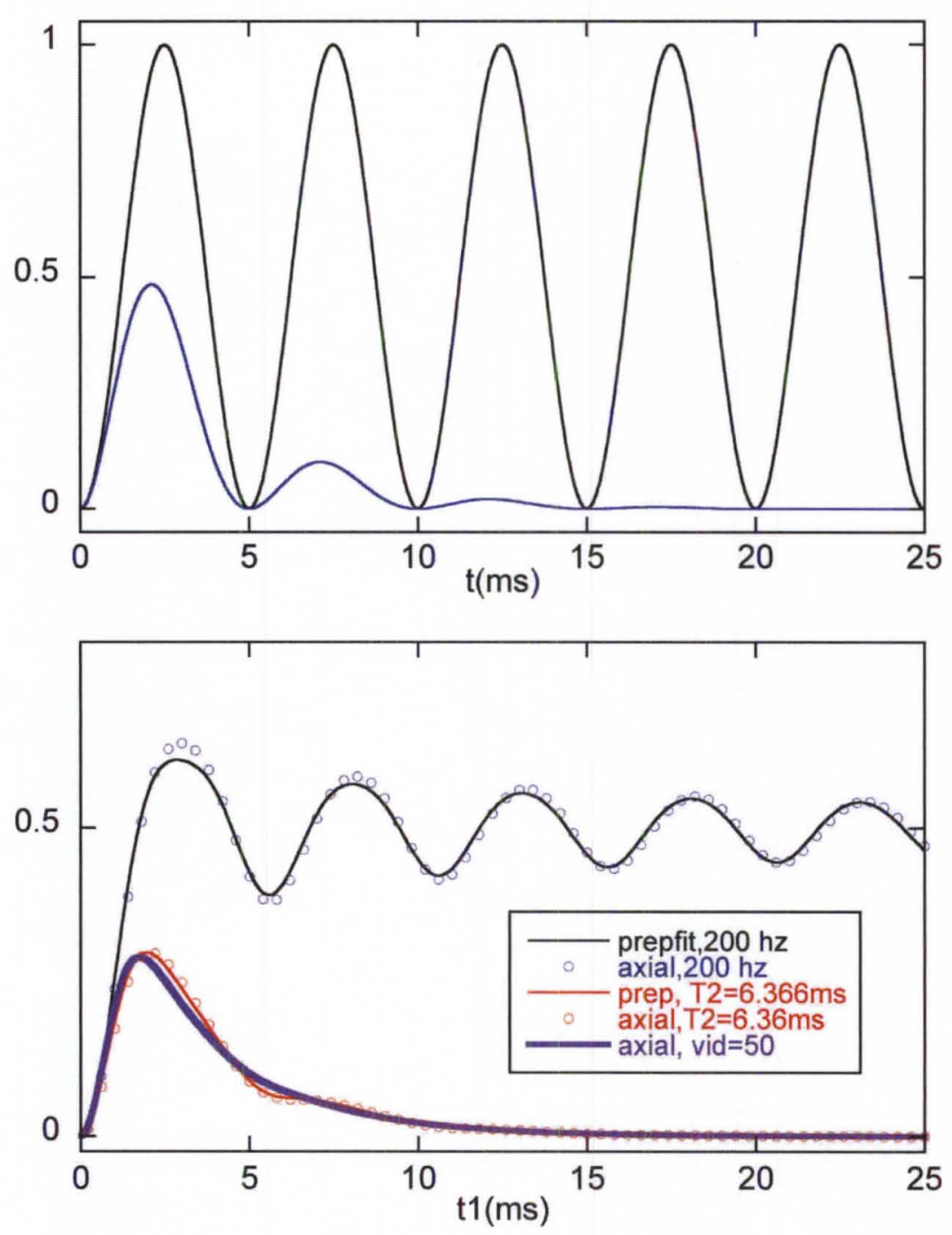

Figure 3.9. Illustration of the effect of relaxation and distribution of quadrupolar couplings on preparation curves. Top, no distribution of couplings: black curve, no relaxation, blue curve, $\mathrm{T}_{2}^{\text {surf }}=6.36 \mathrm{~ms}$. Bottom, distribution of quadrupolar couplings, with $v_{q} 200 \mathrm{~Hz}$ : black curve, no relaxation and eq. 1, circles, no relaxation and eq. 2; with relaxation $\mathrm{T}_{2}^{\text {surf }}=6.36 \mathrm{~ms}$, and linewidth $\Delta \mathrm{v}=1 \mathrm{~Hz}$, eq. 1 - red solid curve, eq. 2 - red circles; purple solid curve - assumption of axially symmetric coupling distribution and linewidth $\Delta v=50 \mathrm{~Hz}$ ) on the simulated preparation curve. 


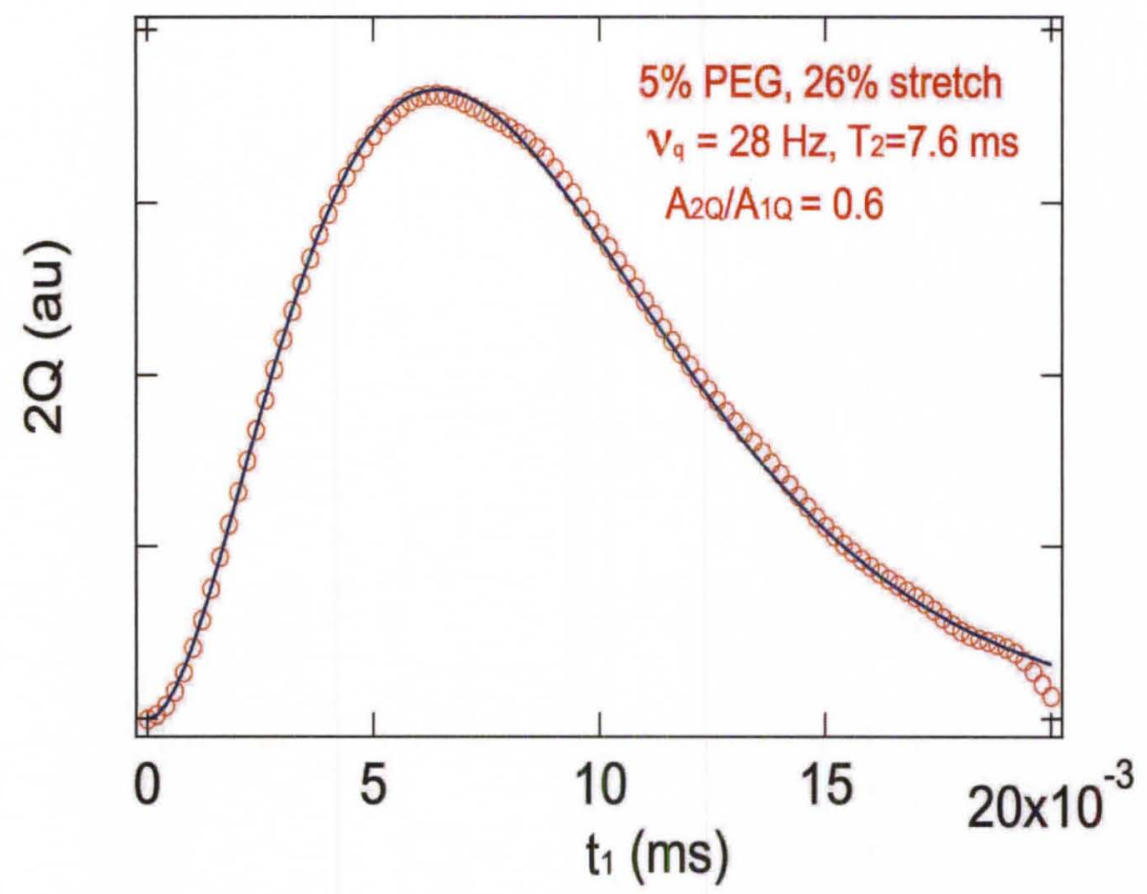

Figure 3.10. An experimental $2 Q$ preparation curve obtained from native, hydrated elastin fibers. Each data point (red circle) is the $2 Q$ filtered signal intensity at the indicated preparation/conversion time, $t_{1}$. The blue solid curve is a least squares fit of the data to the fitting function $S_{2 Q}$, eq. 2 .

An example of an experimental preparation curve obtained from the native, hydrated elastin fibers, is shown in Figure 3.10. Fitting the preparation curve with eq. 2 requires three parameters: the $2 Q$ signal amplitude, $A_{2 Q}$, the residual quadrupolar frequency, $\omega_{\mathrm{q}}=2 \pi \mathrm{v}_{\mathrm{q}}$, and $\mathrm{T}_{2}^{\text {surf }}$, the $1 \mathrm{Q}$ transverse relaxation time. It is observed that the fit is not unique unless $T_{2}^{\text {surf }}$ is determined independently. 


\subsection{Conclusions}

Based on numerical simulations, a new pulse sequence and phase cycle were designed. Experimental tests of the $2 Q$ filter show excellent $1 Q$ silencing. This required a 64-step phase cycle that overcomes limitations such as imprecise transmitter phase shifts or pulse widths. Other improvements are also implemented. The constant time evolution removes relaxation effects during this period and allows clean separation of coherences by order. This was used to confirm $2 Q$ filter efficiency. Alignment and purge increase $S / N$ due to the detection of absorptive signal rather than dispersive signals resulting in significant enhancement of $\mathrm{S} / \mathrm{N}$ and reduction of acquisition time.

A new analysis of preparation experiments was developed that accounts for the distribution of quadrupolar couplings. Fitting the preparation curve allows quantitative determination of the size of the $2 Q$ signal (the amount of ordered water), and the residual coupling (a measure of the degree to which the surface water is ordered). 


\section{CHAPTER 4}

\section{MECHANISM OF ELASTICITY IN ELASTIN}

\subsection{Introduction}

The mechanism of elasticity in materials like rubber is based on the conformational entropy of the polymer [118-120]. It is also generally assumed that the driving force for reversible recoil in elastin is an increase in entropy associated with contraction. This is consistent with the fact that the fiber contracts if temperature is increased. However, dry elastin is brittle and inelastic and the presence of solvent is crucial for elasticity. In this heterogeneous system of protein and solvent, the entropy sources are the protein, the solvent or both. The canonical view is that elastin is like rubber, i.e. stretch reduces the protein's conformational entropy. The alternative view [121-124] is based on the hydrophobic effect and proposes that stretch increases water exposed hydrophobic surface area thereby increasing solvent order and lowering solvent entropy. This is the basis of the hydrophobic effect which is generally regarded as the dominant thermodynamic driving force in protein folding [125]. While somewhat counterintuitive, the latter view is consistent with both molecular

dynamics simulations [124, 126] and calorimetric experiments on elastin [122] 
that show that the large amount of heat released upon stretch is consistent with the known thermodynamic properties of the hydrophobic effect.

To evaluate the relative contributions of backbone and solvent ordering to elastin's recoil mechanism, we use a combination of ${ }^{13} \mathrm{C}$ and $2 \mathrm{Q}$ filtered ${ }^{2} \mathrm{H}$ SSNMR of stretched and unstretched elastic fibers. This approach depends on the unique capability of SSNMR to accurately determine the averaging effect of molecular reorientation on anisotropic nuclear spin interactions; specifically ${ }^{13} \mathrm{C}$ chemical shielding and ${ }^{2} \mathrm{H}$ nuclear quadrupolar coupling.

NMR can be used to evaluate molecular ordering by the degree to which molecular motions decrease the observable anisotropy, known as the inhomogeneous broadening, that results from anisotropic chemical shielding or quadrupolar coupling. In the first approximation, the ratio of the observed broadening, $\Delta \sigma^{\text {obs }}$, in the soft solid to the broadening observed in a rigid solid (or the soft solid under appropriate conditions such as low temperature), $\Delta \sigma^{\text {static }}$, is the order parameter, $S^{(2)}$. Values of $I S^{(2)} I$ vary from 0 (for isotropic reorientation) to 1 (for a rigid solid). The lower limit that order parameters can be determined in weakly ordered systems is the ratio of the homogeneous linewidth, i.e., the linewidth in the absence of anisotropic interactions to the magnitude of the static anisotropy. Magnitudes of shielding and quadrupolar coupling anisotropies in rigid solids are summarized in Table 4.1. 
Table 4.1. Chemical shielding and quadrupolar coupling anisotropies for different nuclei.

\begin{tabular}{ccc}
\hline Spin & CS anisotropy (11.7 T) & Q anisotropy \\
\hline${ }^{1} \mathrm{H}$ & $10 \mathrm{kHz}$ & 0 \\
${ }^{13} \mathrm{C}$ & $15 \mathrm{kHz}$ & 0 \\
${ }^{15} \mathrm{~N}$ & $8 \mathrm{kHz}$ & 0 \\
${ }^{2} \mathrm{H}$ & $1.5 \mathrm{kHz}$ & $200 \mathrm{kHz}$ \\
${ }^{17} \mathrm{O}$ & $10 \mathrm{kHz}$ & $6 \mathrm{MHz}$ \\
\hline
\end{tabular}

Shielding anisotropy of ${ }^{13} \mathrm{C}$ and ${ }^{15} \mathrm{~N}$ are useful for studying backbone (polymer) ordering and the large quadrupolar coupling anisotropies of ${ }^{2} \mathrm{H}$ and ${ }^{17} \mathrm{O}$ are useful for detecting very weakly ordered water molecules. In this dissertation, the results using ${ }^{13} \mathrm{C}$ and ${ }^{2} \mathrm{H}$ spectroscopy are presented. Applications of ${ }^{15} \mathrm{~N}$ require isotopically labeled material and ${ }^{17} \mathrm{O}$ NMR introduce additional technical complications. Thus, ${ }^{15} \mathrm{~N}$ and ${ }^{17} \mathrm{O}$ experiments are indicated as future directions. To evaluate the hydrophobic effect based mechanism of elastin's recoil, the signal from water that does not interact with the protein (water that reorients isotropically and has no residual quadrupolar coupling) must be separated from the signal from water at the protein surface (water that is weakly and transiently ordered). To do this, we use the special properties of multiple quantum ${ }^{2} \mathrm{H} N M R$, chapter 3.

To explore the mechanism of elasticity by NMR, we also consider quantitative aspects of how the molecular properties observed in the NMR experiments are related to elastin's mechanical function. To make this 
connection, we use thermodynamics and establish the relation between NMR observables and thermodynamic entropy. Connecting the NMR experiment to Boltzmann entropies, $S_{B}$ [127], has been discussed by several groups [128-131] and we adapt that procedure to our experiments in section 4.2.

These studies not only allow us to relate spectroscopic NMR parameters to macroscopic, mechanical function, but are also fundamental in more general contexts. First, the hydrophobic effect is the dominant thermodynamic force for protein folding [132] and it remains poorly understood at the molecular level. Insofar as elastin is the most hydrophobic protein known and its hydrophobicity can be altered by mechanical manipulation, it is an excellent system for studying the hydrophobic effect at the molecular level. Transient interaction of solvent at the surface and diffusional exchange with surrounding solvent are inherent features of the hydrophobic effect [132] and a unique advantage of NMR compared to other experimental methods in this context is that time-dependent processes can be studied while the system is at equilibrium. Second, magnetic resonance imaging (MRI) based on MQ NMR is an emerging technique $[97,104$, 110-112] and the underlying physical chemistry of how water interacts with a soft tissue and can be used to efficiently generate $M Q$ coherence is fundamental to obtaining image contrast.

The experiments described below are unique since we investigate both solvent and protein in native material (fully hydrated, elastic protein). 


\subsection{Theory}

To connect what is measured in the NMR experiments (residual anisotropies) with thermodynamic entropy, we adapt the approach used by the groups of Wand, Kay and Palmer [128-131] that is based on the generalized order parameter, $S^{2},[129]$ determined from relaxation experiments. This line of reasoning is readily adapted to the task here where a somewhat different order parameter, $S^{(2)}$, is determined. $S^{(2)}$ and the rotational contribution to Boltzmann entropy, $S_{B}$, are related to the normalized orientational distribution function, $P^{\mathrm{eq}}(\Omega)$, by the following expressions,

$$
S^{(2)}=\frac{v_{q}}{v_{q}^{\text {static }}}=\int P_{2}(\cos \theta) P^{e q}(\theta) \sin \theta d \theta
$$

and

$$
S_{B}=-n R \int_{0}^{\pi} P^{e q}(\theta) \ln P^{e q}(\theta) \sin \theta d \theta
$$

In the above, $v_{q}$ is the observed coupling, $v_{q}^{\text {static }}$ is the static quadrupolar coupling (for ${ }^{2} \mathrm{H} v_{\mathrm{q}}^{\text {static }}=216 \mathrm{kHz}$ in ice [133]) and $P_{2}(\cos \theta)=\frac{1}{2}\left(3 \cos ^{2} \theta-1\right)$ is the second Legendre polynomial. For simplicity, we have assumed solvent dynamics are axially symmetric and thus a function only of the polar angle $\theta$. Expanding $P^{e q}(\Omega)$ in a complete set of orthogonal functions, $P_{1}(\cos \theta)[134]$,

$$
P^{e q}(\theta)=\sum S^{(1)} P_{1}(\cos \theta) \text { and } S^{(1)}=\int_{0}^{\pi} P_{1}(\cos \theta) P^{e q}(\theta) \sin \theta d \theta
$$


indicates the following problem: to determine entropy, all $S^{(1)}$ are required, but only $S^{(2)}$ is available from the NMR experiment. It is possible to overcome this problem by assuming a model distribution function that depends on a single parameter. Several groups $[128-129,134-136]$ have assumed the following model: The "bond vector" (here the unique axis of the quadrupolar coupling tensor) rotationally diffuses in a cone of semiangle $\theta_{0}$,

$$
P^{e q}(\theta)=\left\{\begin{array}{c}
\left(1-\cos \theta_{0}\right)^{-1}, 0 \leq \theta \leq \theta_{0} \\
0, \theta>\theta_{0}
\end{array}\right\} .
$$

This is the "diffusion in a cone" model [136] illustrated in (Figure 4.1) using a ${ }^{2} \mathrm{H}_{2} \mathrm{O}$ molecule. Two limiting cases are a well ordered solid, $\theta_{0}=0$, and an isotropic fluid, $\theta_{0}=\pi$. This model has been justified [129] by molecular dynamics simulations for $\theta_{0}>15^{\circ}$ and is expected to provide an upper limit for $S_{B}$ [128]. With this distribution function, the order parameter and entropy are,

$$
S^{(2)}=\frac{\cos \theta_{0}}{2}\left(1+\cos \theta_{0}\right) \text { and } S_{B}=n R \ln \left(1-\cos \theta_{0}\right) \text {, }
$$

where $\mathrm{n}$ is the number of moles of the solvent and $\mathrm{R}$ is the gas constant. The assumption is that it is equally probable for the "bond vector" to explore any orientation within the shaded area (Figure 4.1). For the case of interest here, the solvent is weakly ordered, i.e., $\theta_{0}$ approaches $\pi$. Therefore it is convenient to define the small angle $\theta_{1}=\pi-\theta_{0}$. The "bond vector" in this case can occupy any orientation on the sphere except within the cone of semiangle $\theta_{1}$. 


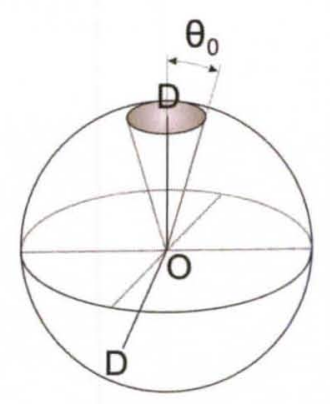

Well ordered

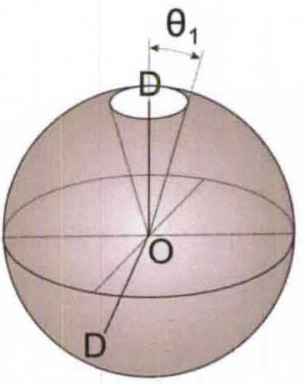

Weakly ordered

Figure 4.1. The diffusion in a cone model for a water molecule. The D-O bond explores orientations within the shaded area. The narrow cone of semiangle $\theta_{0}$ is for well ordered water and the sphere excluding the cone of semiangle $\theta_{1}=\theta_{0}-\pi$ is for weakly ordered water.

With Taylor series expansion in $\theta_{1}$, the following expressions are obtained for the order parameter and entropy:

$$
\mathrm{S}^{(2)}=-\frac{1}{4} \theta_{1}^{2} \text { and } \mathrm{S}_{\mathrm{B}}=\mathrm{nR} \ln 2-\frac{1}{4} \mathrm{nR} \theta_{1}^{2},
$$

where the first term of $S_{B}$ describes an isotropic water with order parameter $S^{(2)}=0$. Combining the above and taking into account the cancellation of the RIn2 factor (since the total amount of water in the sample does not change with stretch) gives a simple expression for estimating the entropy change from the NMR data:

$$
\Delta S_{B}=R\left(n_{12} S_{12}^{(2)}-n_{11} S_{11}^{(2)}\right)
$$




\subsection{Materials and methods}

Elastin fibers from bovine neck ligament, prepared by the neutral extraction method [41], were obtained from Elastin Products Co., Owensville, Missouri.

Static ${ }^{13} \mathrm{C}$ NMR spectra of relaxed and $\sim 50 \%$ stretched elastin fiber were used to evaluate polymer residual shielding anisotropies. Spin-lattice relaxation (longitudinal relaxation) times, $\mathrm{T}_{1}$, of different carbon types (backbone, $\mathrm{C}^{\prime}$ and $\mathrm{C}_{\alpha}$, and side-chain methyl groups, $\mathrm{CH}_{3}$ ) were also measured using the standard inversion recovery experiment with sequence $180^{\circ}-T-90^{\circ}-\mathrm{FID}$. In this experiment, equilibrium longitudinal magnetization is first inverted (from $+z$ to $-z$ ) by a $180^{\circ}$ pulse and magnetization evolves back due to spin-lattice relaxation during the recovery time, $T$. Subsequently, a $90^{\circ}$ pulse converts the longitudinal magnetization into detected transverse magnetization and the experiment is repeated with different $\mathrm{T}$ values. The exponential rise of peak intensity, $\mathrm{S}$, as a function of recovery time was least squares fit to the function $S=S_{0}\left[1-(1+a) \exp \left(-t / T_{1}\right)\right]$ to determine $T_{1}$.

The fraction of water in the elastin sample that is ordered and the degree to which that water is ordered are determined with the $M Q$ preparation experiment described in chapter 3 (Figure 3.4) in combination with Hahn echo experiments. Regarding the former, a constant time, $t_{2}=40 \mu \mathrm{s} \ll \mathrm{T}_{2}^{2 \mathrm{Q}} \approx 10 \mathrm{~ms}$, was used to minimize attenuation of $2 Q$ signal through relaxation during the $2 \mathrm{Q}$ evolution period. Absorption mode spectra were obtained with a purge time, $\mathrm{T}^{\prime}=15 \mathrm{~ms}$, which was found to be sufficient to eliminate antiphase 
coherence. The 2D preparation experiments were analyzed using the following function introduced in chapter 3 ,

$$
S_{2 Q}\left(t_{1}\right)=A_{2 Q} \exp \left(-2 t_{1} / T_{2}^{\text {surf }}\right) \int_{0}^{\pi} \sin ^{2}\left(\frac{1}{2} \omega_{q}\left(3 \cos ^{2} \theta-1\right) t_{1}\right) \sin \theta d \theta
$$

This function has three parameters: $A_{2 Q}, T_{2}^{\text {surf }}$ and $\omega_{q}$ that are fit to experiment. $A_{2 Q}$ is proportional to the ordered water fraction in the sample, $\omega_{q}$ is the residual quadrupolar coupling of the ordered water and $T_{2}^{\text {surf }}$ accounts for spin relaxation during the preparation and conversion periods of length $t_{1}$. This experiment is shown schematically in Figure 4.2, (b) and compared with a simple 1Q experiment, Figure 4.2, (a). 


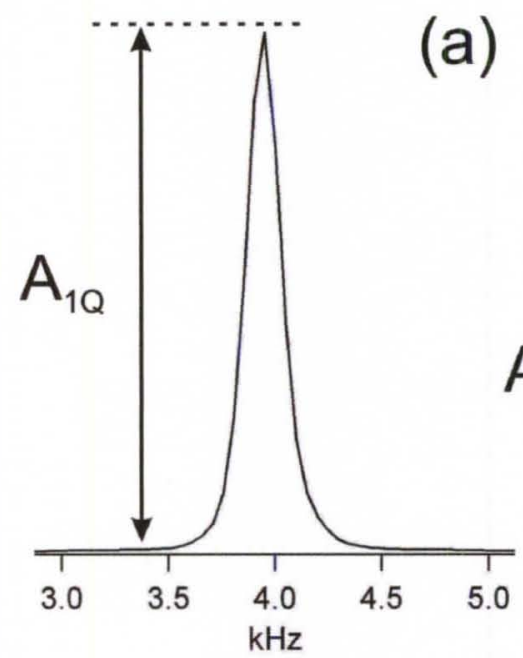

(b)



Figure 4.2. Parameters for the evaluation of the fraction of ordered water in the sample and the residual quadrupolar coupling from $1 Q$ (a) and $2 Q$ (b) experiments. The signal from all water in the sample, $A_{1 Q}$, is the peak intensity of the 1Q signal evaluated from a Hahn echo $\left(\mathrm{T} \ll T_{2}\right)$ spectrum, (a). In the 2D preparation experiment, (b), peak intensities are plotted as a function of preparation/conversion time, $t_{1}$. 
The $1 Q$ signal intensity, $A_{1 Q}$, is proportional to the total amount of water in the sample and $A_{2 Q}$ is proportional to the number of ordered waters, i.e., spins with non-zero quadrupolar coupling. The proportionality constant is a property of the NMR instrument. Thus, it is the same in both cases so the ratio $A_{2 Q} / A_{1 Q}$ is the fraction of waters in the sample that have non-zero quadrupolar coupling. While $A_{1 Q}$ is directly available from the $1 Q$ spectrum (a Bloch decay or a Hahn echo), extraction of $A_{2 Q}$ from the $2 Q$ experiment is more difficult. This is because the $1 Q$ transverse relaxation rate is larger than the quadrupolar coupling, $1 / T_{2} \geq v_{q}$, i.e., the quadrupolar splitting is unresolved. Moreover, preparation/conversion times, $t_{1}$, are also comparable to $v_{q}$. This is why $S_{2 Q}$, Figure 4.2 , (b), depends on both relaxation and quadrupolar coupling. The upper dotted curve shows the diminution of signal from relaxation and the lower curves (experiment, red, and fit, blue) show the combined effects of relaxation and coherent evolution under the quadrupolar interaction. The parameters $\omega_{q}, A_{2 Q}$ and $T_{2}^{\text {surf }}$ are highly correlated and, even in cases when the data has high $S / N$, unique values of all three parameters are not determined by this data alone. Consequently, to obtain unique parameter values, the fit is constrained to the value of $T_{2}^{\text {surf }}$ measured independently.

Three different $T_{2}$ relaxation times were measured, $T_{2}^{\text {surf }}, T_{2}^{\text {bulk }}$ and, for comparison, the relaxation time of pure solvent, $T_{2}^{\text {pure }} \cdot T_{2}^{\text {bulk }}$ is the composite transverse relaxation time for all observed water in the elastin sample ("bulk" 
designates all the solvent). This and $T_{2}^{\text {pure }}$ were measured with a standard Hahn echo experiment, $90^{\circ}-\mathrm{T} / 2-180^{\circ}-\mathrm{T} / 2-\mathrm{FID},[137] . \mathrm{T}_{2}^{\text {surf }}$ is the relaxation time of the solvent that interacts with the protein surface ("surf" designates solvent interacting with the protein surface). $T_{2}^{\text {surf }}$ was measured with a $2 Q$ filtered version of Hahn echo sequence, i.e., replacing the usual $90^{\circ}$ pulse in the Hahn echo with the $2 Q$ filtration sequence described in chapter 3 . Values of $T_{2}^{\text {suff }}$ measured in hydrated elastin at different hydration levels and with added polyethylene glycol (PEG, $\langle M W\rangle=6 \mathrm{kDa}$ ) are shown in Table 4.2. In most cases, the assumption of transverse relaxation characterized by a single exponential time-constant, $T_{2}^{\text {surf }}$, is verified. The exceptions are for pure aqueous solvent where the relaxation rate is more rapid at longer times, $t_{1}>10 \mathrm{~ms}$. Enhanced, non-exponential relaxation is a well-known consequence of diffusion or spin exchange and likely represents the fact that solvent is only transiently ordered at the protein surface [138]. That said, for times $t_{1}<10 \mathrm{~ms}$, the effects of relaxation on the $M Q$ experiment are quantitatively consistent with single exponential relaxation, i.e., eq. 1 , and our model operationally accounts for this. 
Table 4.2. $\mathrm{T}_{2}^{\text {surf }}$ relaxation times as a function of PEG and the amount of solvent.

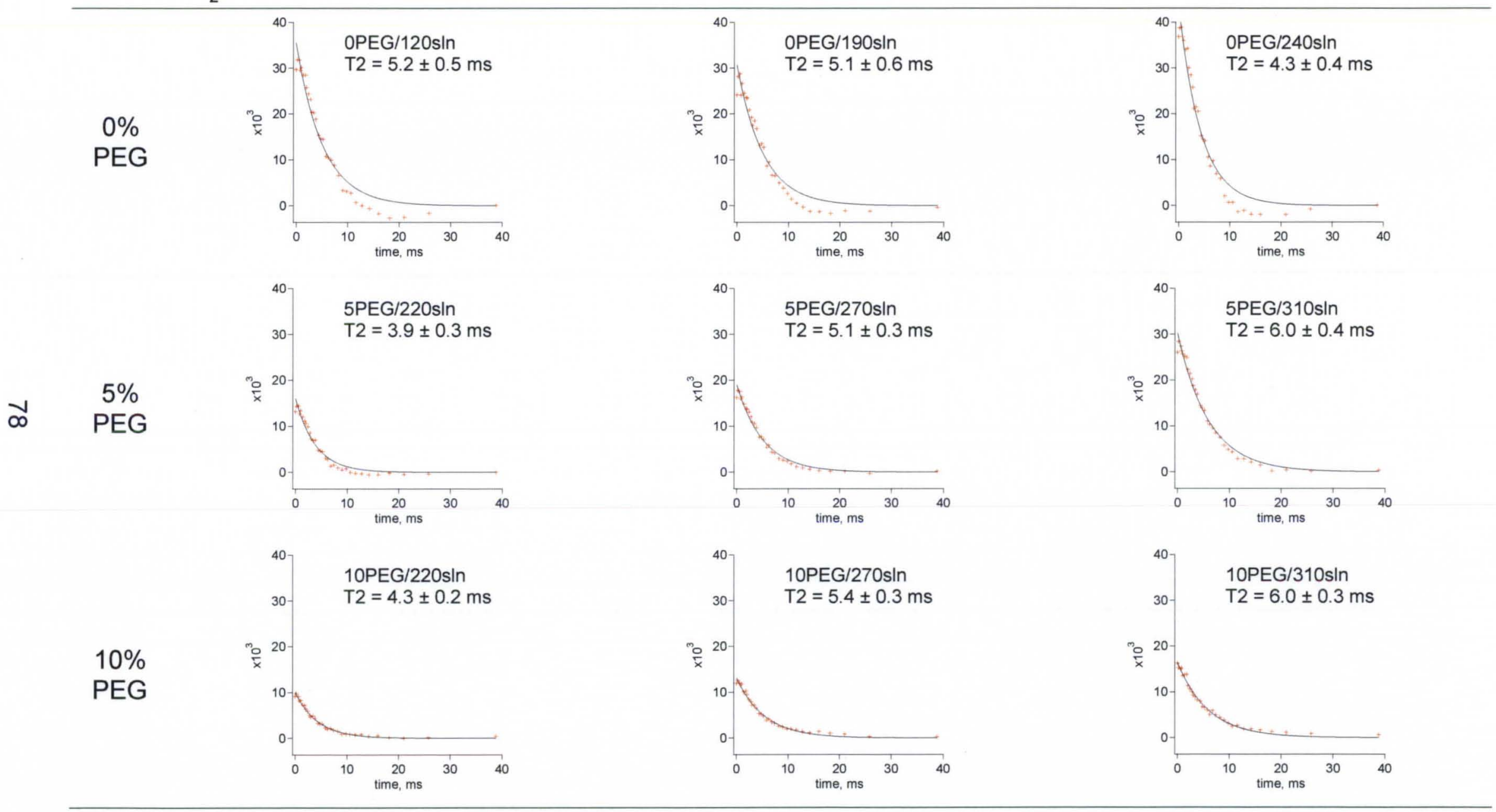


Table 4.3. Dependence of ${ }^{2} \mathrm{H} 2 \mathrm{Q}$ signal size of elastin fiber on the amount of solvent in the sample at constant PEG (5\% PEG) and constant stretch (39\% stretch).

$220 \% \mathrm{w}_{\text {sol }} / \mathrm{w}_{\text {pro }}$

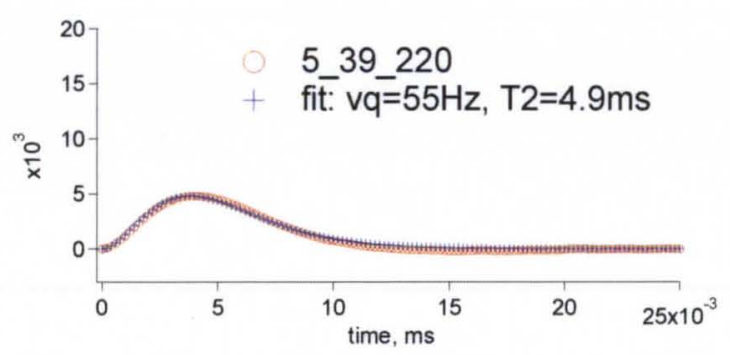
$270 \% \mathrm{w}_{\text {sol }} / \mathrm{w}_{\text {pro }}$

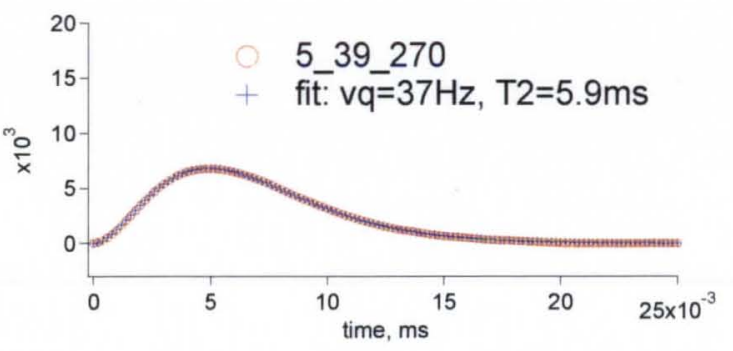

$310 \% \mathrm{w}_{\mathrm{sol}} / \mathrm{w}_{\mathrm{pro}}$

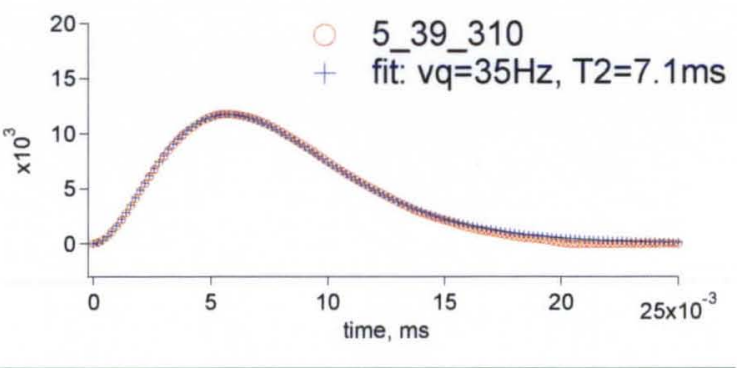


The data in Table 4.2 also shows that results are dependent on the \% water in the sample, defined as

$$
\% w_{\text {sol }} / w_{\text {pro }}=\frac{\text { weight of hydrated sample }- \text { weight of dry sample }}{\text { weight of dry fiber }} \times 100 \% \text {. }
$$

Table 4.3. The above results show, that in this two-phase system, maintaining the solvent/protein mass (weight) ratio constant is essential for valid comparison of the samples when another variable such as temperature or stretch is varied. Results presented in this dissertation were obtained at constant sample hydration level of $\sim 300 \% \mathrm{w}_{\mathrm{so}} / \mathrm{w}_{\mathrm{pro}}$.

To systematically vary the thermodynamic activity of water while holding $w_{\text {sol }} / w_{\text {pro }}$ constant, we varied the amount of PEG in the solvent [139]. The choice of PEG with $\langle M W\rangle=6 \mathrm{kDa}$ was dictated by having $\langle\mathrm{MW}\rangle>1 \mathrm{kDa}$ to prevent interactions of PEG with intrafibrillar water. It has been shown that a mixed $\mathrm{PEG} / \mathrm{H}_{2} \mathrm{O}$ solvent does not significantly alter elastin's microscopic structure [139]. Adding PEG decreases the amount of water available for hydrating elastin without decreasing the solvent volume.

Samples for stretch dependent experiments used single, weighed elastin fibers $(\sim 1 \mathrm{~mm} \times 10 \mathrm{~mm}$ and $\sim 5-10 \mathrm{mg})$ glued to plastic (G10) rods, equilibrated against solvent to a known mass and then sealed in a $3 \mathrm{~mm}$ NMR tube as shown in Figure 4.3. Fibers were stretched by moving the rods and securing their positions with either parafilm or epoxy glue. 


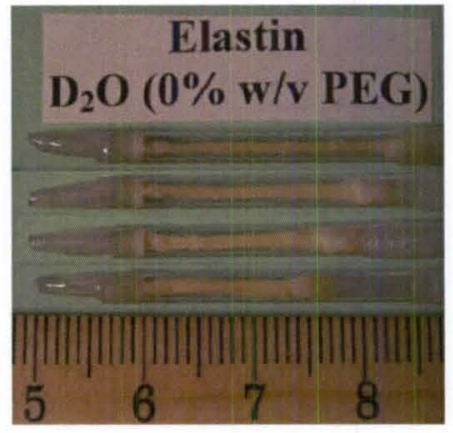

Figure 4.3. Elastin samples at a different stretch.

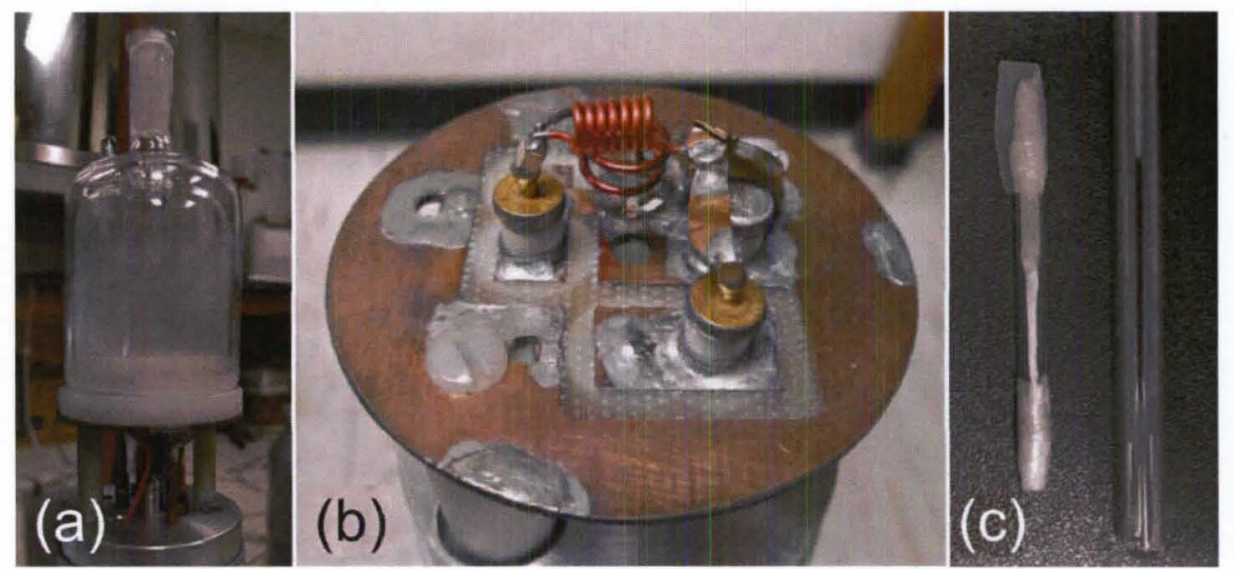

Figure 4.4. Experimental apparatus (a) Probe with Dewar for temperature controlled experiments. (b) Double resonance ${ }^{1} \mathrm{H} / I^{2} \mathrm{H}$ or ${ }^{1} \mathrm{H} /{ }^{13} \mathrm{C}$ static sample NMR probe. (c) Hydrated elastin sample in a $3 \mathrm{~mm}$ NMR tube.

Spectra were obtained with a home built $11.7 \mathrm{~T}$ instrument and the ${ }^{1} \mathrm{H} /{ }^{2} \mathrm{H}$ probe, which can also be used as ${ }^{1} \mathrm{H} /{ }^{13} \mathrm{C}$ probe after soldering appropriate capacitors mounted on the probe bulkhead [140]. The probe has a ${ }^{2} \mathrm{H} 90^{\circ}$ pulse width of $2 \mu \mathrm{s}\left(\sim 150\right.$ watts) and $\mathrm{a}{ }^{13} \mathrm{C} 90^{\circ}$ pulse width of $3 \mu$ s ( 110 watts). The probe circuit is shown in Figure 4.5. 


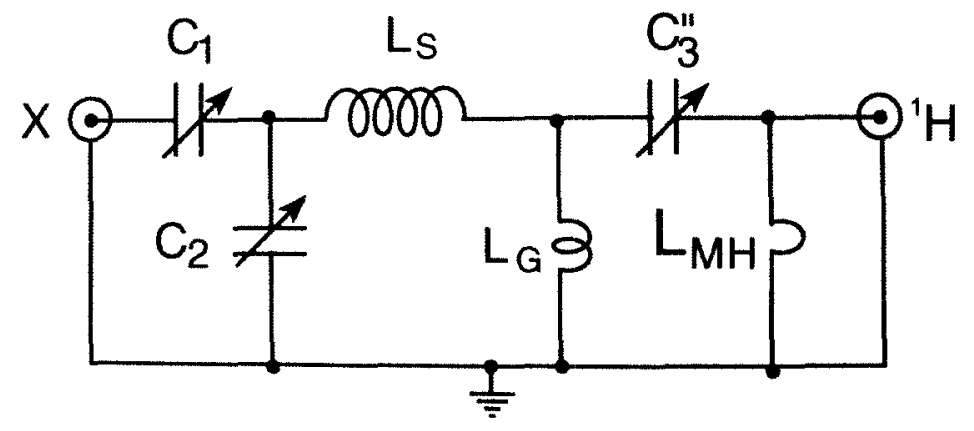

Figure 4.5. Double resonance circuit.

The sample coil, $L_{s}$, is tuned to both the ${ }^{1} \mathrm{H}$ and $X\left({ }^{2} \mathrm{H}\right.$ or $\left.{ }^{13} \mathrm{C}\right)$ frequencies. To assure optimum power transfer between the probe and the transmitter or receiver, the input impedances of the circuit (Figure 4.5) are matched to $50 \Omega . C_{1}$ and $\mathrm{C}_{2}$ are the matching and tuning capacitors on the low frequency channel, $\mathrm{X}$, while $C_{3}^{\prime \prime}, L_{G}$ and $L_{M H}$ are the tuning capacitor, the ground inductance and the matching inductance on the high frequency channel, ${ }^{1} \mathrm{H}$. The approximate tuning and matching conditions are given in Table 4.4.

Table 4.4. Tuning and matching conditions for the double resonance probe.

Channel Tuning Matching

$$
\begin{array}{lll}
\mathrm{X} & \omega_{\mathrm{X}}=\frac{1}{\sqrt{\left(\mathrm{C}_{1}+\mathrm{C}_{2}\right) \mathrm{L}}} & \frac{1}{\mathrm{Q} \omega_{\mathrm{X}}^{3} \mathrm{C}_{1}^{2} \mathrm{~L}}=\mathrm{Z}_{0} \\
{ }^{1} \mathrm{H} & \omega_{{ }_{1 H}}=\frac{1}{\sqrt{\mathrm{C}_{3}^{\prime \prime}\left(\mathrm{L}^{\prime}+\mathrm{L}_{M H}\right)}} & \frac{\mathrm{Q}^{\prime} \omega_{1} L_{M H}^{2}}{\mathrm{~L}^{\prime}}=Z_{0}
\end{array}
$$

Note: $Q$ and $Q^{\prime}$ are the quality factors for $X$ and ${ }^{~} H$ channels respectively; $L=L_{S}+L_{G}$ and $L^{\prime}=L_{S} L_{G} /\left(L_{S}+L_{G}\right) ; Z_{0}=50 \Omega$. Two approximations were used: (1) losses of $L$ and $L^{\prime}\left(R=\omega_{X} L / Q\right.$ and $R^{\prime}=\omega_{1} L^{\prime} / Q^{\prime}$ respectively) are small for high $Q$ sample coil and (2) stray capacitance and inductance can be ignored. 
To determine any possible contribution of amide deuterons to the observed $2 \mathrm{Q}$ "signals", we used experiments (see results) in which amide-solvent exchange was either quenched $\left(\mathrm{pH} 3,5^{\circ} \mathrm{C}\right)$ or made rapid $\left(\mathrm{pH} 7,37^{\circ} \mathrm{C}\right)$. The arrangement for doing this is shown in Figure 4.6 and the buffers used were as follows: $\mathrm{pH} 7$ and pH $3100 \mathrm{mM}$ Na phosphate in ${ }^{2} \mathrm{H}_{2} \mathrm{O}$, and pH 3, $100 \mathrm{mM} \mathrm{Na}$ phosphate in $\mathrm{H}_{2} \mathrm{O}$.
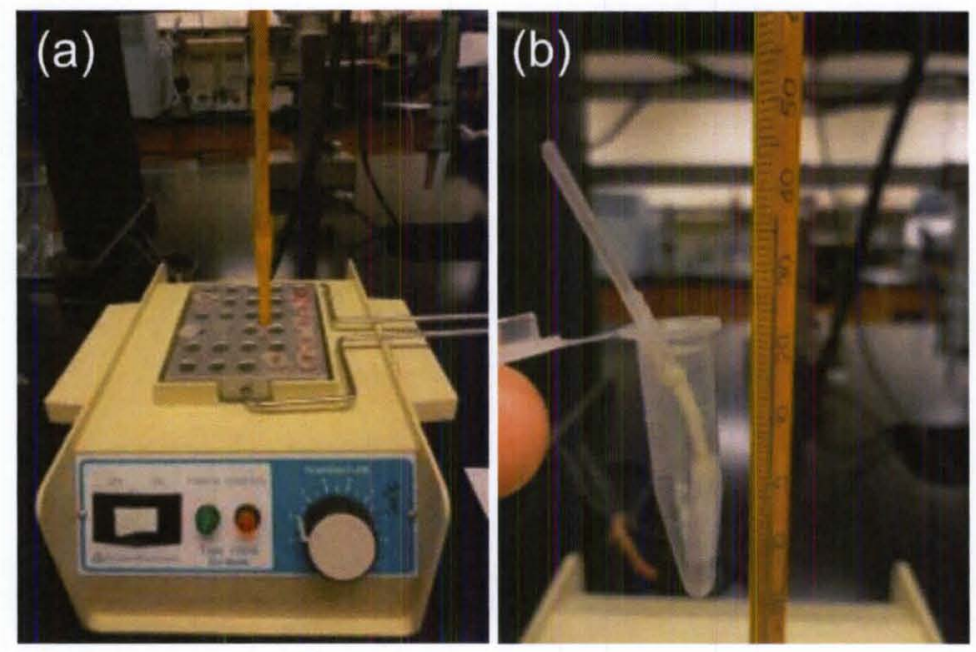

Figure 4.6. Setup for the H-D exchange experiment: (a) heat block with elastin samples; (b) close up for the elastin sample exchanging with the solvent in the Eppendorf tube at $37^{\circ} \mathrm{C}$.

\subsection{Results: ${ }^{13} \mathrm{C}$ spectroscopy of the polymer}

The hypothesis that recoil is driven by conformational entropy of the stretched polymer, as in rubbers, implies that the residual shielding anisotropy in ${ }^{13} \mathrm{C}$ NMR spectrum increases with stretch.

\section{Residual shielding}

To investigate this assumption, we obtained the natural abundance, static ${ }^{13} \mathrm{C}$ spectra of fully hydrated elastin in relaxed and stretched states (Figure 4.7). These spectra are a refinement of published result [63]. Spectral resolution, 
given that the spectra are obtained without magic angle spinning, is remarkable. This is because residual shielding anisotropies are small and approach that of isotropic fluids. Although the static shielding anisotropy, $\Delta \sigma^{\text {static }}$, of a typical peptide carbonyl is $150 \mathrm{ppm}$ [63], the breadth of the carbonyl peak, $\Delta \sigma^{\text {obs }}$, in hydrated elastin, as seen from the results in Figure 4.7, is no more than $10 \mathrm{ppm}$.
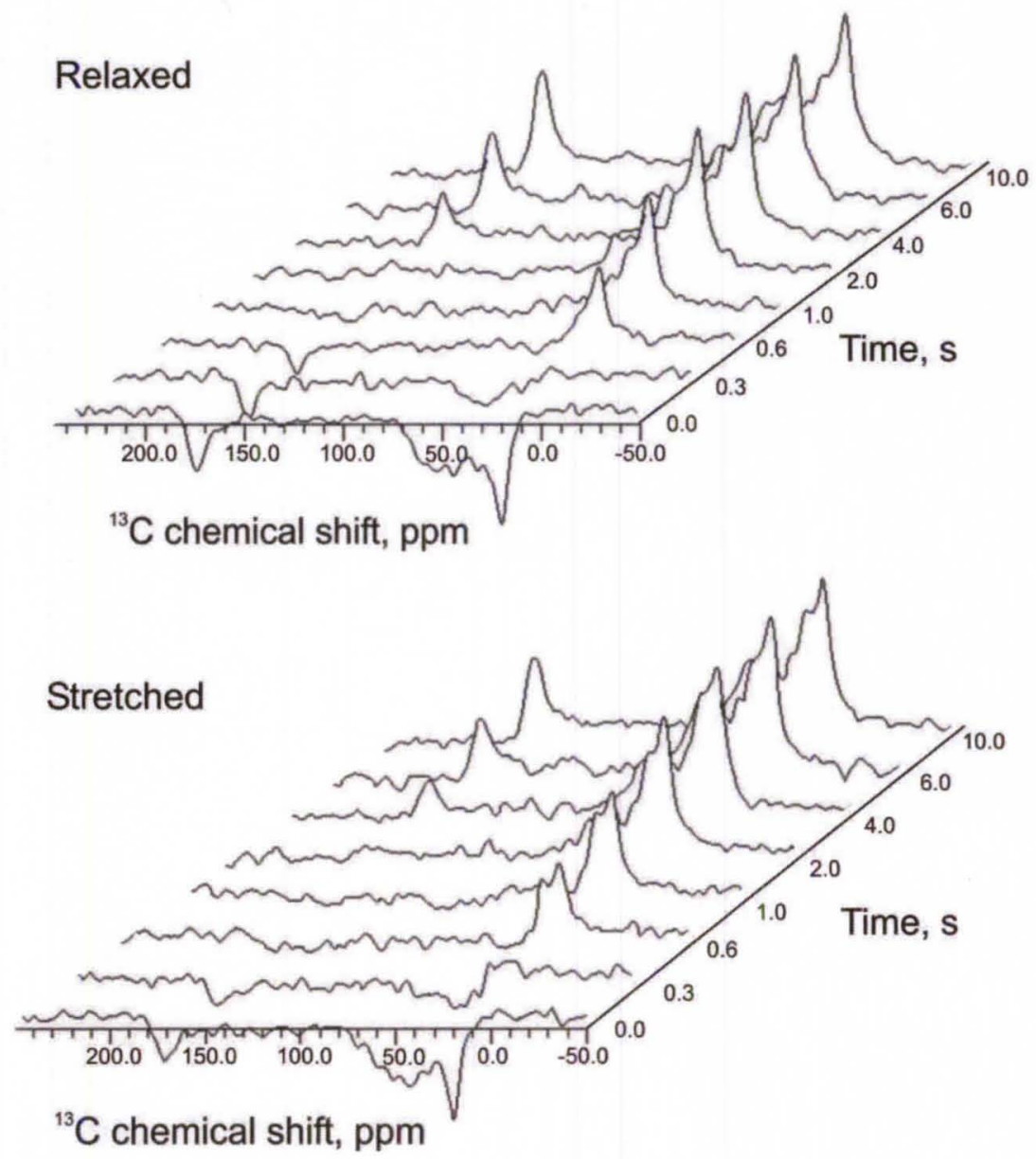

Figure 4.7. Static ${ }^{13} \mathrm{C}$ NMR spectra of hydrated elastin.

Comparison of the ${ }^{13} \mathrm{C}$ spectra for stretched and relaxed elastin shows no significant change in residual shielding anisotropies with stretch (the lineshapes are almost identical in both cases). Evaluation of stretch induced changes in 
$\Delta \sigma^{\text {obs }}$ is subject to two limitations here. At the small residual anisotropies observed, the contributions from homogeneous broadening originating from relaxation and a range of isotropic chemical shifts are not negligible. Thus, while we cannot exclude the possibility of stretch induced ordering of the polymer chain, our results show no evidence for it. Obtaining accurate shielding anisotropies will require experimental refinements, like using model elastin peptides with simpler sequences and/or labeling of individual atomic sites. For

example, one can label the nitrogen atoms with ${ }^{15} \mathrm{~N}$, which has a smaller range of isotropic chemical shifts, and use 2D heteronuclear correlation experiments to better resolve carbon and nitrogen signals.

\section{$T_{1}$ Studies}

Longitudinal relaxation times, $T_{1}$, were also determined from inversion recovery experiments for stretched and relaxed elastin fiber (Figure 4.7). Each spectrum in Figure 4.7 is taken at certain recovery time $\mathrm{T}$ (indicated on the time axis). Determined $T_{1}$ values are summarized in Table 4.5.

Table 4.5. Longitudinal relaxation time $T_{1}$ for stretched and relaxed elastin fiber.

\begin{tabular}{ccc}
\hline Site & Stretched $\mathrm{T}_{1}$, sec. & Relaxed $\mathrm{T}_{1}$, sec. \\
\hline $\mathrm{C}^{\prime}$ & $5.30 \pm 0.80$ & $4.10 \pm 0.40$ \\
$\mathrm{C}_{\mathrm{a}}$ & $1.29 \pm .22$ & $1.20 \pm .14$ \\
$\mathrm{CH}_{3}$ & $0.53 \pm 0.04$ & $0.52 \pm 0.03$ \\
\hline
\end{tabular}

The new result from these studies is the observation of little or no significant change (within the given standard errors) in $T_{1}$ for backbone $\left(C^{\prime}\right.$ and $C_{a}$ ) and side-chain methyl groups. 
4.5. Results: MQ NMR studies of the solvent

If solvent is involved in the entropy change that drives the reversible recoil of elastin, then it must interact with the protein and, consequently, is at least transiently ordered on the protein surface. To evaluate the possible contribution of solvent to the mechanism of recoil, the amount of ordered water in the sample (the $2 Q$ signal intensity, $A_{2 Q}$ ) and the strength of solvent ordering (the residual quadrupolar coupling, $v_{q}$ ) were measured in response to stretch, hydration and temperature.

\section{Source of the ${ }^{2} H$ NMR signals}

Before presenting these results, we confirm that the observed NMR signal originates from the solvent and not from exchanged labelled amides. This is important since equilibrating elastin in ${ }^{2} \mathrm{H}_{2} \mathrm{O}$, exchange labels both the solvent and the protein at amide groups. 1D, 2Q filtered spectra shown in Figure 4.8 verify that the contribution of protein amides to the $2 Q$ signal is negligible. To do this, we have taken advantage of the capability of acquiring $2 Q$ filtered spectra in a time $(\sim 1$ minute) that is short compared to the exchange time $\left(\mathrm{t}_{1 / 2} \sim 20\right.$ minutes) of protein amides at $\mathrm{pH} 3$ and $5^{\circ} \mathrm{C}$ [141].

The three spectra in Figure 4.8 were obtained from a sample following: (a) exhaustive exchange against ${ }^{2} \mathrm{H}_{2} \mathrm{O}$ with equilibration at $\mathrm{pH} 3$, (b) back exchange for 6 minutes against ${ }^{1} \mathrm{H}_{2} \mathrm{O}$ buffer at $\mathrm{pH} 3,5^{\circ} \mathrm{C}$ and (c) re-exchange against ${ }^{2} \mathrm{H}_{2} \mathrm{O}$. 
Since spectrum (b) was obtained in a time insufficient to significantly exchange the amide deuterons, the 200-fold attenuation of the signal in spectrum (b) relative to spectrum (a) confirms that the $2 Q$ signal in (a) arises exclusively from rapidly exchangeable solvent. Spectrum (c) confirms that solvent back exchange is rapid.

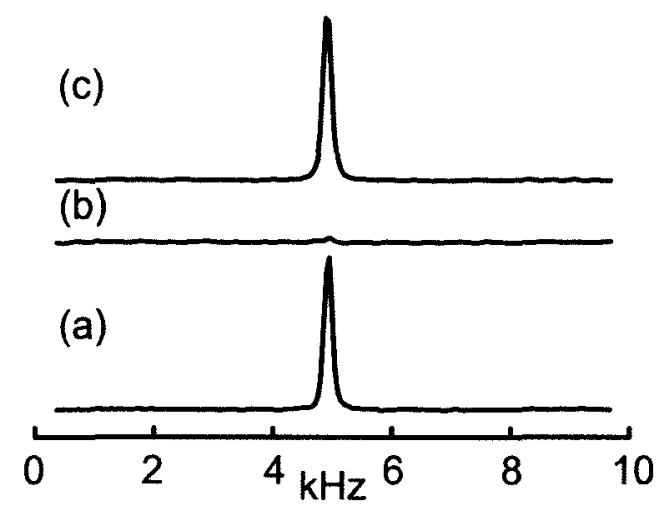

Figure 4.8. Ordered water signal after exchange against ${ }^{2} \mathrm{H}_{2} \mathrm{O}(\mathrm{a})$, ${ }^{1} \mathrm{H}_{2} \mathrm{O}\left(\right.$ b) and ${ }^{2} \mathrm{H}_{2} \mathrm{O}$ (c).

Therefore, we have confirmed that contribution of the protein amides to the $2 Q$ signal is negligible and the use of $2 Q$ filtered ${ }^{2} H$ NMR (Figure 3.4) is legitimate for the detection of anisotropic solvent.

\section{Effect of stretch and hydration on relaxation times}

$\mathrm{T}_{2}^{\text {pure }}$ and $\mathrm{T}_{2}^{\text {bulk }}$ were measured with stepwise increases in the fiber length by as much as $50 \%$ and at three PEG levels, $0 \%, 5 \%$ and $10 \%$.

For comparison with the elastin samples, $T_{2}$ for the deuterated solvent alone, $T_{2}^{\text {pure }}$, was measured at room temperature without and with the addition of PEG, Table 4.6. The effect of PEG on pure solvent is simply to increase solution viscosity and the expected decrease in $T_{2}^{\text {pure }}$ is observed. 
Table 4.6. $T_{2}^{\text {pure }}$ relaxation time of pure solvent as a function of PEG content.
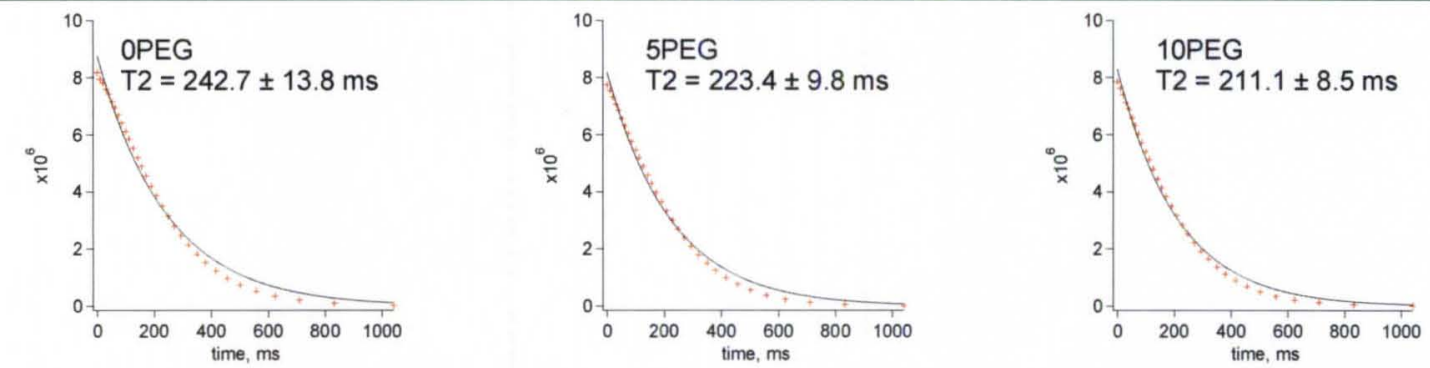

$T_{2}^{\text {pure }}$ values (Table 4.6) yield rotational correlation times (see Appendix $C$ ), $T_{c}$, of 4.6 ps for pure ${ }^{2} \mathrm{H}_{2} \mathrm{O}$ and 5.3 ps in $10 \%$ w/v PEG. These were obtained using standard expressions, Appendix $\mathrm{B}$, for quadrupolar relaxation and the known ${ }^{2} \mathrm{H}$ quadrupolar coupling constant for liquid ${ }^{2} \mathrm{H}_{2} \mathrm{O}$ at room temperature, $v_{q}=245 \mathrm{kHz}$ [142]. These values are a small overestimation of the expected value, $T_{c}=2.4 p s$ [143], because samples were not degassed and dissolved paramagnetic oxygen causes efficient relaxation. Increasing the viscosity by addition of PEG leads to slower molecular reorientation and increased correlation times since the correlation time is proportional to the reciprocal of the diffusion constant and the Stokes-Einstein relation has a reciprocal dependence of the diffusion constant on viscosity. Overall, the correlation time is directly proportional to the viscosity.

Experimental values of $T_{2}^{\text {bulk }}$ as a function of fiber stretch and PEG level are shown in Table 4.7. The data are well described by single exponential relaxation time with the indicated error limits. 
Table 4.7. $T_{2}^{\text {bulk }}$ relaxation time as a function of stretch and PEG.

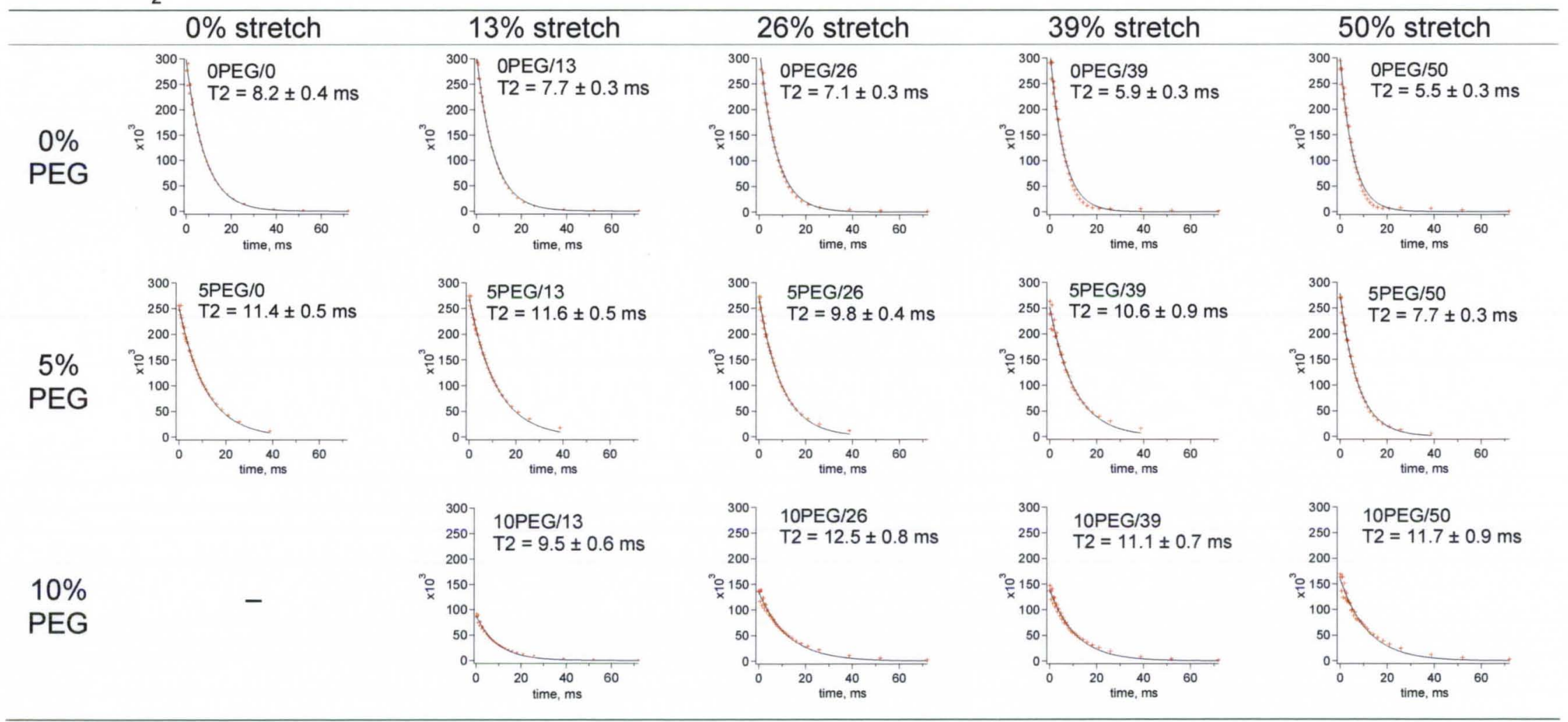


Compared to pure solvent value, $\mathrm{T}_{2}^{\text {pure }} \sim 200 \mathrm{~ms}, \mathrm{~T}_{2}^{\text {bulk }} \sim 10 \mathrm{~ms}$ indicates a significant slowing of the effective water reorientational rate. Important trends in the data are (a) increasing stretch decreases $\mathrm{T}_{2}^{\text {bulk }}$, (b) addition of PEG at fixed stretch leads to an increase in $T_{2}^{\text {bulk }}$ (opposite to the effect in pure solvent) and (c) signal intensity at zero echo delay does not change with stretch or PEG concentration if the fiber masses (0 \% PEG (11 mg), $5 \%$ PEG (9 mg) and $10 \%$ PEG (5 mg)) are taken into account. Observations (a) and (c) are discussed below in the context of $T_{2}^{\text {surf }}$ data and (c) shows that the fraction of the total sample water that is observed in this NMR experiment does not vary significantly with either stretch or with the addition of PEG. We conclude that the observed bulk water signal likely encompasses all of the water in the sample.

The $2 Q$ filtered data from which values of $T_{2}^{\text {surf }}$ were determined as a function of stretch and PEG content are given in Table 4.8. The experiment was made at the optimal preparation/conversion time, separately determined in the 2D preparation experiment. 
Table 4.8. $\mathrm{T}_{2}^{\text {surf }}$ relaxation time as a function of stretch and PEG.

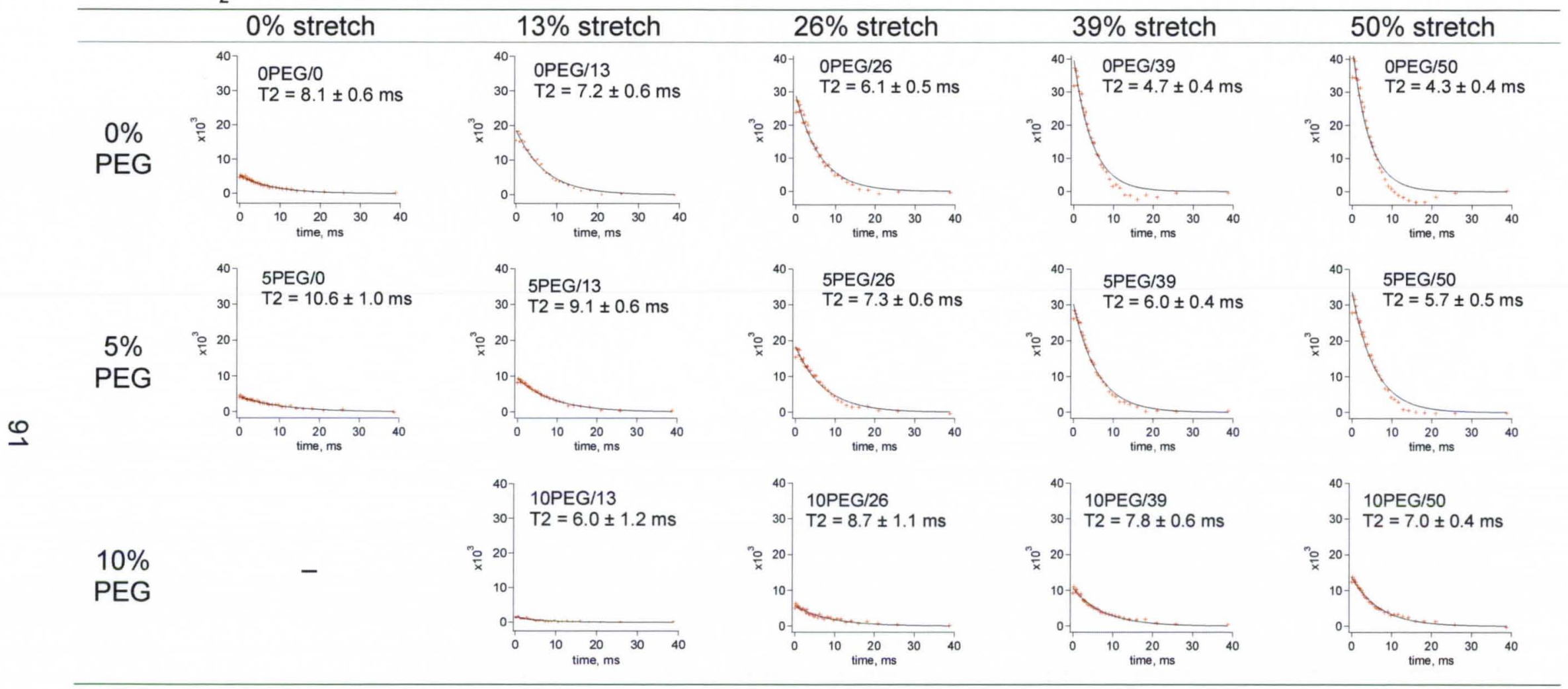


In contrast to the unfiltered experiment (Table 4.7) in which all of the water in the sample is observed, the size of the $2 Q$ signal increases substantially, $\sim$ 10-fold, with stretch (Table 4.8). Thus, a larger fraction of the total water in the sample passes through the $2 Q$ filter when the elastin is stretched, i.e., the size of the ordered water "pool" increases with stretch. This, and the fact that $\mathrm{T}_{2}^{\text {bulk }}$ and $\mathrm{T}_{2}^{\text {surf }}$ values are somewhat different, shows that bulk and ordered water do not fully exchange on the time scale of this experiment. To put this conclusion in perspective, consider the distance water diffuses during the $M Q$ experiment in relation to the known microstructural features of elastin fibrils. The root mean square diffusional distance, $\sqrt{2 \mathrm{Dt}} \approx 0.7 \mu \mathrm{m}$ (using the self-diffusion constant for water, $D \approx 2.23 \times 10^{-9} \mathrm{~m}^{2} / \mathrm{s}[144]$ and a typical preparation time, $t=10 \mathrm{~ms}$ ) is not larger than a typical fibril diameter, $1-3 \mu \mathrm{m}$ [145], or the interfibrillar spaces, $1-10 \mu \mathrm{m}[145]$. Consistent with the increase in the ordered water signal intensity with stretch, addition of PEG decreases sample elasticity and significantly decreases the $2 Q$ filtered signal intensity. Thus, the amount of interfibrillar water contributing to the $2 Q$ signal decreases with PEG and $T_{2}^{\text {bulk increases as }}$ observed. An important observation is that the signal size in the $2 Q$ filtered experiment is far more sensitive monitor of the changes in the sample with stretch and hydration (PEG) then are changes in either $T_{2}^{\text {bulk }}$ or $T_{2}^{\text {surf }}$.

\section{Variation of the ordered solvent fraction and residual coupling with stretch}

The change in $2 Q$ signal with stretch observed in the measurements of $\mathrm{T}_{2}^{\text {surf }}$, are confirmed in the $2 \mathrm{D}$ preparation experiments, Table 4.9. In combination 
with the relaxation results (above), two important parameters are also determined: the fraction of water that is ordered in the sample, $A_{2 Q} / A_{1 Q}$, and the residual quadrupolar coupling, $v_{q}$. On the time scale of the $2 \mathrm{D}$ preparation experiment, $\sim 10 \mathrm{~ms}$, we reasonably assume that there is diffusional exchange between the two water pools. Consequently, fits of the $2 Q$ signal intensities to the parameters $A_{2 Q} / A_{1 Q}$ and $v_{q}$ are constrained to the range of $T_{2}$ values from $\mathrm{T}_{2}^{\text {surf }}$ to $\mathrm{T}_{2}^{\text {bulk }}$. Parameter ranges determined in this way are significantly larger than parameter standard errors resulting from random errors such as finite $\mathrm{S} / \mathrm{N}$. Consequently, parameter ranges but not standard errors are listed. The $A_{10}$ values used are signal intensities from the $\tau=0$ spectrum of the data set used to determine $T_{2}^{\text {bulk }}$.

For $0 \% \mathrm{w} / \mathrm{v}$ PEG, the fraction of water in the sample that is ordered increases with stretch from $6 \%$ to $73 \%$ and the residual coupling varies only by a small amount, $24-42 \mathrm{~Hz}$. Since $T_{2}^{\text {surf }}$ decreases with stretch, the effect of relaxation is to decrease the $2 Q$ signal intensity. Thus, the observed $\sim 10$-fold increase in $2 Q$ signal can only be explained by an increase in the amount of ordered water. As mentioned earlier, increasing PEG stepwise from $0 \%$ to $10 \%$ $w / v$ is known to reduce elasticity. Thus reduced elasticity also correlates with a decrease in the $2 Q$ signal, $T_{2}^{\text {surf }}$ and $T_{2}^{\text {bulk }}$. Slight increase in quadrupolar coupling with PEG indicates stronger interaction of the solvent with the protein. 
Table 4.9. ${ }^{2} \mathrm{H} 2 \mathrm{Q}$ signal and parameters from preparation curve experiment as a function of stretch and PEG.

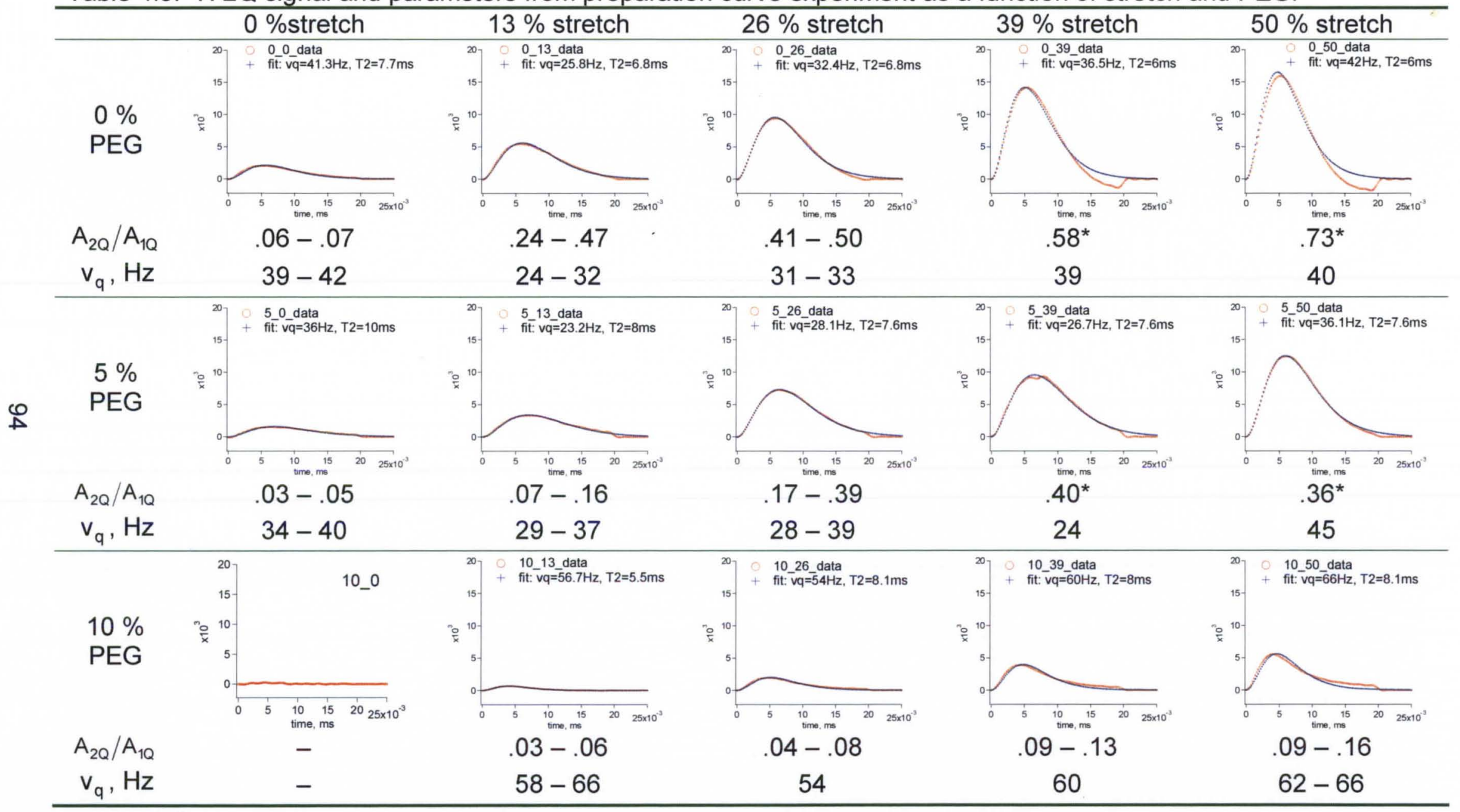

* with $T_{2}^{\text {surf }}, A_{2 Q} / A_{1 Q}>1$ and neglected. 


\section{Effect of temperature on solvent ordering}

Increasing order with temperature is a signature of hydrophobic forces. In all elastomers, the effect of stretch is force generation and it is generally agreed that elastin is an entropic spring, i.e., entropy decreases with stretch, $(\partial \mathrm{S} / \partial \mathrm{I})_{\mathrm{T}}<0$. Consequently, the Maxwell relation, $-(\partial \mathrm{S} / \partial \mathrm{I})_{\mathrm{T}}=(\partial \mathrm{F} / \partial \mathrm{T})_{1}$, predicts that increasing temperature increases the recoil force at fixed length, I. If, the hydrophobic effect is involved in this force, it follows that increased temperature must also increase water ordering. This expectation, contrary to what is expected from thermalization of the water ordering, is confirmed in Figure 4.9.

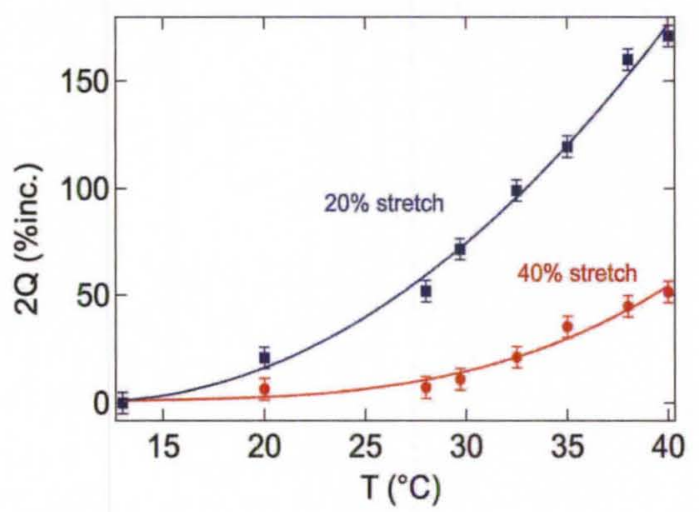

Figure 4.9. Water ordering as function of temperature.

At $20 \%$ and $40 \%$ stretch, the ordered water $2 Q$ signal increases by approximately $200 \%$ and $50 \%$, respectively, as the temperature is increased from $13^{\circ} \mathrm{C}$ to $40^{\circ} \mathrm{C}$. Interestingly, the onset of the effect, from $30^{\circ} \mathrm{C}$ to $40^{\circ} \mathrm{C}$, coincides with the transition temperature for tropoelastin coacervation, $37^{\circ} \mathrm{C}[146]$. Whether or not this is coincidental is a subject of future investigations. 


\subsection{Discussion and conclusions}

The results from ${ }^{13} \mathrm{C}$ NMR reported here show no observable increase in shielding anisotropy of elastin protein backbone carbons upon stretch, i.e. no evidence for ordering of the polymer is seen in this experiment. As noted, small changes in anisotropy are masked by finite linewidths and dispersion in isotropic shifts. Thus, while we cannot exclude the possibility of stretch-induced ordering of the polymer, our results show no support for a recoil mechanism based primarily on polymer entropy. This is consistent with the calorimetric results [122] of Gosline and MD calculations [124, 126] from Daggett, et al., both of which indicate that the hydrophobic effect is the predominant mechanism of recoil. A more recent report based on ${ }^{13} \mathrm{C}$ relaxation $\left(T_{1}\right.$ and $\left.T_{1 p}\right)$ studies [113], however, argues in favor of a polymer configurational entropy based mechanism. In our view, these authors have made four incorrect assumptions in interpreting their results. First, stretch-induced changes in relaxation times were not significantly larger than the indicated error limits. Second, the correlation times determined from the relaxation data made ad hoc assumptions about the size of dipolar couplings rather than using accepted spin coupling parameters $\left({ }^{13} \mathrm{C}\right.$ and ${ }^{1} \mathrm{H}$ gyromagnetic ratios and $\mathrm{C}-\mathrm{H}$ distances). Third, the stretch-induced decrease in polymer correlation times so obtained were interpreted as the reciprocal oscillator frequency of an undamped, harmonic oscillator. Undamped harmonic motions are far too high in frequency to cause spin relaxation and are a poor model for large amplitude reorientational motions about chemical bonds [147] which are better described as diffusive or jump-like [148]. Finally, interpreting a decrease in 
correlation times, i.e., an increase in dynamical rates, as an increase in entropy is not necessarily valid since entropy is an equilibrium property and depends only on the equilibrium distribution of orientations and not on the rates at which different orientations or configurations are sampled. In contrast, the properties emphasized in this study, residual shielding anisotropy and quadrupolar coupling, are functions of the equilibrium distribution of orientations, $P^{\mathrm{eq}}(\Omega)$, and independent of dynamical rates.

That elastomers can operate on the basis of interfacial forces was first suggested by Weis-Fogh and Andersen in 1970 [121]. Consider also that the dominant driving force of elastin's recoil is closely related to the dominant force of protein folding. More specifically, elastic recoil from stretched to relaxed state is similar to the folding of a protein from a fully extended polypeptide to the native, folded state; experiments that can now be performed using pulling experiments based on atomic force microscopy. These experiments show that only a modest portion $(\sim 20 \mathrm{kT})$ of the total work $(\sim 170 \mathrm{kT})$ done by a single polyubiquitin protein on folding from a fully extended polymer is due to the protein's configurational entropy [149]. From the heterogeneity of folding pathways, these authors [149] concluded the existence of an additional driving force of the protein folding. MD simulations indicated that the "remainder of the work, $\sim 150 \mathrm{kT}$, must be mainly due to hydrophobic interactions". Insofar as, extension of elastin fibers by $\sim 50 \%$ is a much smaller conformational change than complete unfolding of a protein, we reasonably expect that elastin's configuration entropy change is only 
a modest contribution to the recoil force. This finding supports our hypothesis that elastin's recoil has a significant contribution from hydrophobic forces.

In the context of interfacial forces between a polymer and solvent, we note that the experimental results reported here show large stretch induced changes compared to, for example, changes in shielding anisotropy or relaxation rates observed for the polymer. It is also instructive to compare the ${ }^{2} \mathrm{H}_{2} \mathrm{O}$ swollen elastin experiments reported here to seminal ${ }^{2} \mathrm{H}$ NMR experiments of Samulski and coworkers on a rubber, polyisoprene, swollen with a hydrophobic, deuterated solvent $\left(\mathrm{CDCl}_{3}, \mathrm{C}_{6} \mathrm{D}_{6}\right.$, etc.) [150-152]. These experiments are the basis of stretched gel based NMR experiments for determining residual dipole couplings. In the rubber and stretched gel experiments, no residual coupling is observed in the absence of stretch, however, splitting appears and increases as the sample is stretched and the signal intensity remains constant. In elastin $/{ }^{2} \mathrm{H}_{2} \mathrm{O}$, relaxed material has a non-zero coupling, the coupling is approximately constant with stretch and the fraction of solvent ordered increases with stretch. These differences make a clear distinction between a hydrophobic solvent in a rubber matrix and a hydrophobic protein, elastin, in water.

The rubber and gel experiments were interpreted by Samulski and coworkers as uniaxial ordering of the polymer network along the stretch axis. The solvent (deuterochloroform, for example) senses the local ordering and an exchange averaged coupling with fixed intensity is observed since the rates of solvent translational diffusion and exchange at the polymer surface are rapid on the NMR timescale. In elastin, we observe a residual coupling in unstretched 
material from either single fibers or randomly oriented bundles of fibers. We conclude that the solvent ordering is the result of a transient interaction with the randomly oriented protein matrix. Since the fraction of water that has nonzero quadrupolar coupling is small compared to 1 in unstretched material, complete exchange averaging does not occur. With stretch, the fraction ordered increases but change in ordering is less significant. Insofar as the water is a probe also of local ordering of the protein matrix, this does not occur with elastin stretch.

Qualitatively, the results presented here show that water in elastin is ordered upon fiber stretch. An important quantitative question follows: Is the entropy change from this solvent ordering a significant part of the thermodynamic driving force to account for elastin's mechanical recoil? To answer this, we obtain an estimate of the contribution of the water to the free energy change using the simple theory described in section 4.2 ,

$$
\Delta S_{B}=R\left(n_{12} S_{12}^{(2)}-n_{11} S_{11}^{(2)}\right),
$$

and the experimental results presented above. In the above, 11 and 12 are relaxed and stretched fiber lengths and $n$ is the number of moles of ordered water obtained from the NMR data in Table 4.9. For $10 \mathrm{mg}$ elastin fiber hydrated with $\sim 24 \mathrm{mg}$ we have $n_{12} \approx .88$ mmole and $n_{11} \approx .08$ mmole. When the fiber is stretched from $11=1 \mathrm{~cm}$ to $12=1.5 \mathrm{~cm}$, the ratio $A_{2 Q} / A_{1 Q}$ increases from $\sim .065$ to $\sim .730$ and the coupling is essentially constant at $\sim 40 \mathrm{~Hz}$. Thus, with $\mathrm{v}_{\mathrm{q}}^{\text {static }}=216 \mathrm{kHz}$, we calculate $\mathrm{T} \Delta \mathrm{S}_{\mathrm{B}} \approx 0.74 \mathrm{~mJ}(\mathrm{~T}=300 \mathrm{~K})$ for the stretch. For comparison, this would be equivalent to the total work (free energy change) if the 
fiber is reversibly stretched the same amount by applying the gravitational force from a $15 \mathrm{~g}$ mass. We anticipate that this comparison is reasonable and indicates that the entropic contribution from water ordering is a significant part of the driving force for recoil.

To conclude, we suggest a model elastin recoil that is consistent with the results presented above. Unstretched fibers are rich in convolutions that are formed as a result of a hydrophobic collapse favored by the very hydrophobic protein. This expels water and reduces solvent entropy. Stretching the fiber opens the convolutions, increases solvent exposed surface area and orders water (Figure 4.10).

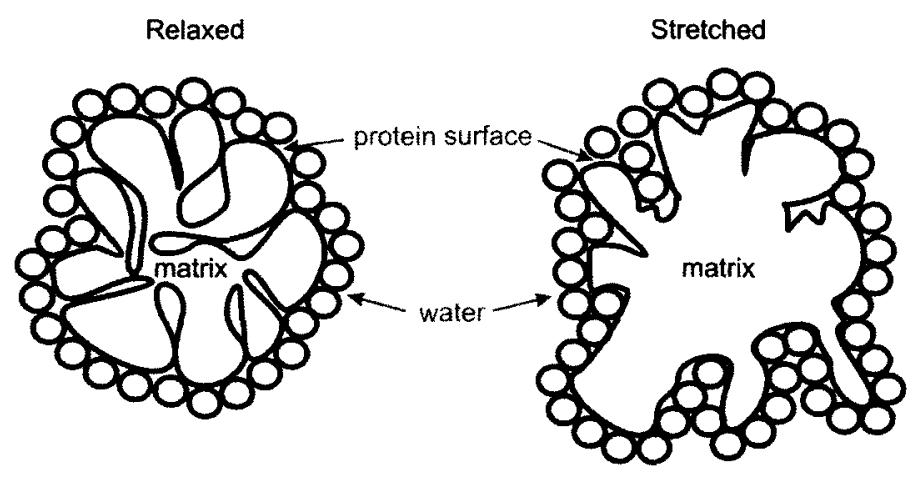

Figure 4.10. Model for elastin's reversible recoil.

Increase in the amount of transiently immobilized water implies decrease of the solvent entropy with stretch. Favorable increase in the solvent entropy drives the recoil of the protein. This model is consistent with all spectroscopic results presented here, including the decrease of $\mathrm{T}_{2}^{\text {surf }}$ relaxation times of surface and bulk water $T_{2}^{\text {bulk }}$ with stretch. 


\section{CHAPTER 5}

\section{CONCLUSIONS AND SUGGESTIONS \\ FOR FUTURE RESEARCH}

Based on the NMR titrations of lamellar TOCL monitored by the $\mathrm{pH}$ dependence of ${ }^{13} \mathrm{C},{ }^{31} \mathrm{P}$ and ${ }^{1} \mathrm{H}$ chemical shifts, we conclude that there is no evidence for the existence of a titratable group with a $\mathrm{pK}_{\mathrm{a}}$ in the range of 4 to 9 . The estimation of the stabilization energy required for raising the $\mathrm{pK}_{\mathrm{a}}$ value of a phosphodiester by approximately 5 units is also in line with this conclusion. In our view, the likely source of contradiction of results from our NMR studies and potentiometric titrations is a difference in phase. Unfortunately, the authors of the structural hypothesis [72] did not provide any details on the phase of $\mathrm{CL}$ in their experiments. Insofar as the lamellar phase is the most interesting for investigation of the biological role of $\mathrm{CL}$, performing potentiometric titration on lamellar liquid crystalline phase $C L$ is suggested.

To refine the tentative assignments of the two peaks in the glycerol region of the HSQC spectrum and distinguish $G_{1}^{\prime}$ and $G_{3}$ peaks explicitly we suggest to use the Heteronuclear Multiple Bond Correlation (HMBC) experiment. ${ }^{1} \mathrm{H} /{ }^{13} \mathrm{C}$ 
HMBC will allow correlation of carbon chemical shifts with hydroxyl proton (in case of $G_{1}^{\prime}$ ) and methine proton (for $G_{3}$ ).

The novel ${ }^{2} \mathrm{H} M Q$ methods devised in this work includes several considerable improvements compared to the generally used methods for surface ordering studies. Inclusion of alignment and purge significantly enhances $\mathrm{S} / \mathrm{N}$ due to the detection of absorptive signal and therefore reduces the time required for the experiments. Spectra with excellent $\mathrm{S} / \mathrm{N}$ can be obtained in experiments that last about one minute using small, single fiber samples. This allows us to perform experiments involving $H / D$ exchange and single fiber samples which are ideal for evaluations of mechanical properties.

The approach for the design of the $2 Q$ pulse sequence and phase cycles for MQ filtration that was successfully applied for ${ }^{2} \mathrm{H}$ can be adapted to any other quadrupolar nucleus. ${ }^{17} \mathrm{O}$ is particularly beneficial in aqueous solvent studies. Transition frequencies and basis operators for $I=5 / 2$ spins are provided in Appendix A. The large number of possible coherences and their interference make the development of the phase cycle for the selection of certain coherences the primary obstacle to overcome. New pulse sequences for ${ }^{17} \mathrm{O}$ studies will be required.

Comparisons between rubber and elastin for the amount of stretch induced changes in ordered solvent and the degree of ordering [152], clearly indicate important differences in their elastic mechanisms. The increase of the amount of ordered solvent with approximately constant quadrupolar coupling upon stretch are distinct from the results for rubber and indicate a significant role 
of the solvent in the elastic mechanism of elastin. Moreover, the increase in solvent ordering with increased temperature, demonstrates the importance of the hydrophobic effect in elastin recoil. From this perspective, it would be interesting, to look at the variations in the amount of ordered solvent and strength of recoil when the hydrophobicity of the polymer is systematically varied. Analogous to proteins, we anticipate that in elastin the thermodynamic propensity for folding/recoil will increase [153] if the protein's hydrophobicity is increased. 


\section{REFERENCES}

1. Chevelkov, V., et al., Comparison of Solid-State Dipolar Couplings and Solution Relaxation Data Provides Insight into Protein Backbone Dynamics. Journal of the American Chemical Society, 2010. 132(14): $p$. 5015-5017.

2. Hackel, C., et al., The trehalose coating effect on the internal protein dynamics. Physical Chemistry Chemical Physics, 2012. 14(8): p. 27272734.

3. Helmus, J.J., et al., Determination of methyl (13)C-(15)N dipolar couplings in peptides and proteins by three-dimensional and four-dimensional magic-angle spinning solid-state NMR spectroscopy. Journal of Chemical Physics, 2008. 128(5).

4. Marulanda, D., et al., Resonance assignments and secondary structure analysis of E-coli thioredoxin by magic angle spinning solid-state NMR spectroscopy. Journal of Physical Chemistry B, 2005. 109(38): p. 1813518145.

5. Igumenova, T.I., et al., Assignments of carbon NMR resonances for microcrystalline ubiquitin. Journal of the American Chemical Society, 2004. 126(21): p. 6720-6727.

6. Schnell, J.R., H.J. Dyson, and P.E. Wright, Structure, dynamics, and catalytic function of dihydrofolate reductase. Annual Review of Biophysics and Biomolecular Structure, 2004. 33: p. 119-140.

7. Lorieau, J.L. and A.E. McDermott, Conformational flexibility of a microcrystalline globular protein: Order parameters by solid-state NMR spectroscopy. Journal of the American Chemical Society, 2006. 128(35): p. $11505-11512$.

8. Scheidt, H.A., et al., Solid-State NMR Spectroscopic Investigation of A beta Protofibrils: Implication of a beta-Sheet Remodeling upon Maturation into Terminal Amyloid Fibrils. Angewandte Chemie-International Edition, 2011. 50(12): p. 2837-2840.

9. Friedman, R., Aggregation of amyloids in a cellular context: modelling and experiment. Biochemical Journal, 2011. 438: p. 415-426. 
10. Jaroniec, C.P., et al., High-resolution molecular structure of a peptide in an amyloid fibril determined by magic angle spinning NMR spectroscopy. Proceedings of the National Academy of Sciences of the United States of America, 2004. 101(3): p. 711-716.

11. Iwata, K., et al., 3D structure of amyloid protofilaments of beta(2)microglobulin fragment probed by solid-state NMR. Proceedings of the National Academy of Sciences of the United States of America, 2006. 103(48): p. 18119-18124.

12. Bertini, I., et al., A New Structural Model of $A$ beta(40) Fibrils. Journal of the American Chemical Society, 2011. 133(40): p. 16013-16022.

13. Judge, P.J. and A. Watts, Recent contributions from solid-state NMR to the understanding of membrane protein structure and function. Current Opinion in Chemical Biology, 2011. 15(5): p. 690-695.

14. Vogel, E.P., et al., Solid-State Nuclear Magnetic Resonance (NMR) Spectroscopy of Human Immunodeficiency Virus gp41 Protein That Includes the Fusion Peptide: NMR Detection of Recombinant Fgp41 in Inclusion Bodies in Whole Bacterial Cells and Structural Characterization of Purified and Membrane-Associated Fgp41. Biochemistry, 2011. 50(46): p. $10013-26$.

15. Koder, R.L., Jr., et al., N-15 solid-state NMR provides a sensitive probe of oxidized flavin reactive sites. Journal of the American Chemical Society, 2006. 128(47): p. $15200-15208$.

16. Frericks, H.L., et al., Magic-angle spinning solid-state NMR of a $144 \mathrm{kDa}$ membrane protein complex: E-coli cytochrome bo(3) oxidase. Journal of Biomolecular Nmr, 2006. 36(1): p. 55-71.

17. Jovanovic, T. and A.E. McDermott, Observation of ligand binding to cytochrome P450-BM-3 by means of solid-state NMR spectroscopy. Journal of the American Chemical Society, 2005. 127(40): p. 1381613821.

18. Cook, G.A. and S.J. Opella, NMR studies of membrane proteins. Methods in molecular biology (Clifton, N.J.), 2010. 637: p. 263-75.

19. Hu, W.G., A. Som, and G.N. Tew, Interaction between Lipids and Antimicrobial Oligomers Studied by Solid-State NMR. Journal of Physical Chemistry B, 2011. 115(26): p. 8474-8480.

20. Potrzebowski, M.J., et al., Phosphorus-31 NMR Spectroscopy of Condensed Matter, in Annual Reports on Nmr Spectroscopy, Vol 72, G.A. Webb, Editor. 2011, Elsevier Academic Press Inc: San Diego. p. 35-114. 
21. Aucoin, D., et al., High-resolution (1)H MAS RFDR NMR of biological membranes. Journal of Magnetic Resonance, 2009. 197(1): p. 77-86.

22. Cruciani, O., et al., Multilamellar Liposomes formed by phosphatidyl nucleosides: An NMR-HR-MAS characterization. Langmuir, 2004. 20(4): p. 1144-1151.

23. Gaede, H.C. and K. Gawrisch, Multi-dimensional pulsed field gradient magic angle spinning NMR experiments on membranes. Magnetic Resonance in Chemistry, 2004. 42(2): p. 115-122.

24. Payne, G.S., et al., Evaluation of P-31 high-resolution magic angle spinning of intact tissue samples. Nmr in Biomedicine, 2006. 19(5): p. 593598.

25. Claypool, S.M. and C.M. Koehler, The complexity of cardiolipin in health and disease. Trends in Biochemical Sciences, 2012. 37(1): p. 32-41.

26. O'Leary, W.M., Wilkinson, S.G., Gram-positive bacteria, in Microbial lipids, C. Ratledge, Wilkinson, S.G., Editor. 1988, Academic press: London. p. 117-201.

27. Wilkinson, S.G., Gram-negative bacteria, in Microbial lipids, C. Ratledge, Wilkinson, S.G., Editor. 1988, Academic Press: London. p. 299-488.

28. Pangborn, M.C., Isolation and purification of a serologically active phospholipid from beefheart. Journal of Biological Chemistry, 1942. 143: p. 247-256.

29. Pangborn, M.C., The composition of cardiolipin. Journal of Biological Chemistry, 1947. 168(1): p. 351-361.

30. LeCocq, J., Ballou, C.E., On the structure of cardiolipin. Biochemistry, 1964. 3: p. $976-980$.

31. Schlame, M., S. Brody, and K.Y. Hostetler, MITOCHONDRIAL CARDIOLIPIN IN DIVERSE EUKARYOTES - COMPARISON OF BIOSYNTHETIC REACTIONS AND MOLECULAR ACYL SPECIES. European Journal of Biochemistry, 1993. 212(3): p. 727-735.

32. Kito, M., et al., DIFFERENCES IN FATTY-ACID COMPOSITION AMONG PHOSPHATIDYLETHANOLAMINE, PHOSPHATIDYLGLYCEROL AND CARDIOLIPIN OF ESCHERICHIA-COLI. Biochimica Et Biophysica Acta, 1972. 260(3): p. 475-\&.

33. Chicco, A.J. and G.C. Sparagna, Role of cardiolipin alterations in mitochondrial dysfunction and disease. American Journal of PhysiologyCell Physiology, 2007. 292(1): p. C33-C44. 
34. Kiebish, M.A., et al., Cardiolipin and electron transport chain abnomalities in mouse brain tumor mitochondria: lipidomic evidence supporting the Warburg theory of cancer. Journal of Lipid Research, 2008. 49(12): $p$. 2545-2556.

35. Seddon, J.M., R.D. Kaye, and D. Marsh, INDUCTION OF THE LAMELLAR-INVERTED HEXAGONAL PHASE-TRANSITION IN CARDIOLIPIN BY PROTONS AND MONO-VALENT CATIONS. Biochimica Et Biophysica Acta, 1983. 734(2): p. 347-352.

36. McKenzie, M., et al., Mitochondrial respiratory chain supercomplexes are destabilized in Barth Syndrome patients. Journal of Molecular Biology, 2006. 361(3): p. 462-469.

37. Pfeiffer, K., et al., Cardiolipin stabilizes respiratory chain supercomplexes. Journal of Biological Chemistry, 2003. 278(52): p. 52873-52880.

38. Gonzalvez, F., et al., Cardiolipin provides an essential activating platform for caspase-8 on mitochondria. Journal of Cell Biology, 2008. 183(4): $p$. 681-696.

39. Vrhovski, B. and A.S. Weiss, Biochemistry of tropoelastin. European Journal of Biochemistry, 1998. 258(1): p. 1-18.

40. McCartney, J.E.M., Heat contraction of elastic tissue. Quart. J. Exp. Physiol., 1914. 7: p. 103-114.

41. Partridge, S.M., Davis, H.F., Adair, S.T., The Chemistry of Connective Tissues. 2. Soluble Proteins Derived From Partial Hydrolysis of Elastin. Biochemical Journal, 1955. 61: p. 11-21.

42. Sage, H., STRUCTURE-FUNCTION-RELATIONSHIPS IN THE EVOLUTION OF ELASTIN. Journal of Investigative Dermatology, 1982. 79: p. S146-S153.

43. Chung, M.I.S., et al., Sequences and domain structures of mammalian, avian, amphibian and teleost tropoelastins: Clues to the evolutionary history of elastins. Matrix Biology, 2006. 25(8): p. 492-504.

44. He, D., et al., Comparative genomics of elastin: Sequence analysis of a highly repetitive protein. Matrix Biology, 2007. 26(7): p. 524-540.

45. Yeo, G.C., F.W. Keeley, and A.S. Weiss, Coacervation of tropoelastin. Advances in Colloid and Interface Science, 2011. 167(1-2): p. 94-103.

46. Wise, S.G. and A.S. Weiss, Tropoelastin. International Journal of Biochemistry \& Cell Biology, 2009. 41(3): p. 494-497. 
47. Wise, S.G., et al., Specificity in the coacervation of tropoelastin: solvent exposed lysines. Journal of Structural Biology, 2005. 149(3): p. 273-281.

48. Sato, F., et al., Distinct steps of cross-linking, self-association, and maturation of Tropoelastin are necessary for elastic fiber formation. Journal of Molecular Biology, 2007. 369(3): p. 841-851.

49. Clarke, A.W., et al., Tropoelastin massively associates during coacervation to form quantized protein spheres. Biochemistry, 2006. 45(33): p. 9989-9996.

50. Swee, M.H., W.C. Parks, and R.A. Pierce, DEVELOPMENTAL REGULATION OF ELASTIN PRODUCTION - EXPRESSION OF TROPOELASTIN PRE-MESSENGER-RNA PERSISTS AFTER DOWNREGULATION OF STEADY-STATE MESSENGER-RNA LEVELS. Journal of Biological Chemistry, 1995. 270(25): p. 14899-14906.

51. Pierce, R.A., C.H. Moore, and M.C. Arikan, Positive transcriptional regulatory element located within exon 1 of elastin gene. American Journal of Physiology-Lung Cellular and Molecular Physiology, 2006. 291(3): p. L391-L399.

52. Petersen, E., F. Wagberg, and K.A. Angquist, Serum concentrations of elastin-derived peptides in patients with specific manifestations of atherosclerotic disease. European Journal of Vascular and Endovascular Surgery, 2002. 24(5): p. 440-444.

53. Tatham, A.S. and P.R. Shewry, Elastomeric proteins: biological roles, structures and mechanisms. Trends in Biochemical Sciences, 2000. 25(11): p. 567-571.

54. Kozel, B.A., et al., Domains in tropoelastin that mediate elastin deposition in vitro and in vivo. Journal of Biological Chemistry, 2003. 278(20): $p$. 18491-18498.

55. Muiznieks, L.D. and F.W. Keeley, Proline Periodicity Modulates the Selfassembly Properties of Elastin-like Polypeptides. Journal of Biological Chemistry, 2010. 285(51): p. 39779-39789.

56. Bochicchio, B. and A. Pepe, Role of Polyproline I/ Conformation in Human Tropoelastin Structure. Chirality, 2011. 23(9): p. 694-702.

57. Rauscher, S., et al., Proline and glycine control protein self-organization into elastomeric or amyloid fibrils. Structure, 2006. 14(11): p. 1667-1676.

58. Kumashiro, K.K., et al., Cooperativity between the hydrophobic and crosslinking domains of elastin. Journal of Biological Chemistry, 2006. 281(33): p. 23757-23765. 
59. Milewicz, D.M., Z. Urban, and C. Boyd, Genetic disorders of the elastic fiber system. Matrix Biology, 2000. 19(6): p. 471-480.

60. Yen, A., et al., ELASTODERMA. Journal of the American Academy of Dermatology, 1995. 33(2): p. 389-392.

61. Mammi, M., L. Gotte, and G. Pezzin, COMPARISON OF SOLUBLE AND NATIVE ELASTIN CONFORMATIONS BY FAR-ULTRAVIOLET CIRCULAR DICHROISM. Nature, 1970. 225(5230): p. 380-381.

62. Perry, A., et al., Observation of the glycines in elastin using $\mathrm{C}-13$ and $\mathrm{N}-15$ solid-state NMR spectroscopy and isotopic labeling. Journal of the American Chemical Society, 2002. 124(24): p. 6832-6833.

63. Pometun, M.S., E.Y. Chekmenev, and R.J. Wittebort, Quantitative observation of backbone disorder in native elastin. Journal of Biological Chemistry, 2004. 279(9): p. 7982-7987.

64. Torchia, D.A. and K.A. Piez, MOBILITY OF ELASTIN CHAINS AS DETERMINED BY C-13 NUCLEAR MAGNETIC-RESONANCE. Journal of Molecular Biology, 1973. 76(3): p. 419-424.

65. Rosenbloom, J., W.R. Abrams, and R. Mecham, EXTRACELLULARMATRIX .4. THE ELASTIC FIBER. Faseb Journal, 1993. 7(13): p. 12081218.

66. Fleming, W.W., C.E. Sullivan, and D.A. Torchia, CHARACTERIZATION OF MOLECULAR MOTIONS IN C-13-LABELED AORTIC ELASTIN BY C13-H-1 MAGNETIC DOUBLE-RESONANCE. Biopolymers, 1980. 19(3): p. 597-617.

67. Kricheldorf, H.R. and D. Muller, SECONDARY STRUCTURE OF PEPTIDES .15. C-13 NMR CP MAS STUDY OF SOLID ELASTIN AND PROLINE-CONTAINING COPOLYESTERS. International Journal of Biological Macromolecules, 1984. 6(3): p. 145-151.

68. Perry, A., et al., Solid-state C-13 NMR reveals effects of temperature and hydration on elastin. Biophysical Journal, 2002. 82(2): p. 1086-1095.

69. Ohgo, K., et al., Wideline Separation (WISE) NMR of Native Elastin. Macromolecules, 2009. 42(22): p. 8899-8906.

70. Haines, T.H., ANIONIC LIPID HEADGROUPS AS A PROTONCONDUCTING PATHWAY ALONG THE SURFACE OF MEMBRANES A HYPOTHESIS. Proceedings of the National Academy of Sciences of the United States of America-Biological Sciences, 1983. 80(1): p. 160-164. 
71. Michel, $\mathrm{H}$. and D. Oesterhelt, ELECTROCHEMICAL PROTON GRADIENT ACROSS THE CELL-MEMBRANE OF HALOBACTERIUMHALOBIUM - EFFECT OF N,N'-DICYCLOHEXYLCARBODIIMIDE, RELATION TO INTRACELLULAR ADENOSINE-TRIPHOSPHATE, ADENOSINE-DIPHOSPHATE, AND PHOSPHATE CONCENTRATION, AND INFLUENCE OF THE POTASSIUM GRADIENT. Biochemistry, 1980. 19(20): p. 4607-4614.

72. Kates, M., et al., PH-DISSOCIATION CHARACTERISTICS OF CARDIOLIPIN AND ITS 2'-DEOXY ANALOG. Lipids, 1993. 28(10): p. 877882.

73. Eble, K.S., et al., TIGHTLY ASSOCIATED CARDIOLIPIN IN THE BOVINE HEART MITOCHONDRIAL ATP SYNTHASE AS ANALYZED BY P-31 NUCLEAR-MAGNETIC-RESONANCE SPECTROSCOPY. Journal of Biological Chemistry, 1990. 265(32): p. 19434-19440.

74. Gomez, B. and N.C. Robinson, Phospholipase digestion of bound cardiolipin reversibly inactivates bovine cytochrome bc(1). Biochemistry, 1999. 38(28): p. 9031-9038.

75. Abramovitch, D.A., D. Marsh, and G.L. Powell, ACTIVATION OF BEEFHEART CYTOCHROME-C-OXIDASE BY CARDIOLIPIN AND ANALOGS OF CARDIOLIPIN. Biochimica Et Biophysica Acta, 1990. 1020(1): p. 3442.

76. Silvius, J.R., Thermotropic phase transitions of pure lipids in model membranes and their modification by membrane proteins, in Lipid-protein interactions, P.C. Jost, Griffith, O.H., Editor. 1982, Willey: New York.

77. Lewis, R., et al., Calorimetric, $X$-ray diffraction, and spectroscopic studies of the thermotropic phase behavior and organization of tetramyristoyl cardiolipin membranes. Biophysical Journal, 2007. 92(9): p. 3166-3177.

78. Oldfield, E., J.L. Bowers, and J. Forbes, HIGH-RESOLUTION PROTON AND C-13 NMR OF MEMBRANES - WHY SONICATE. Biochemistry, 1987. 26(22): p. 6919-6923.

79. Sankaram, M.B., G.L. Powell, and D. Marsh, EFFECT OF ACYL CHAIN COMPOSITION ON SALT-INDUCED LAMELLAR TO INVERTED HEXAGONAL PHASE-TRANSITIONS IN CARDIOLIPIN. Biochimica Et Biophysica Acta, 1989. 980(3): p. 389-392.

80. Gally, H.U., W. Niederberger, and J. Seelig, CONFORMATION AND MOTION OF CHOLINE HEAD GROUP IN BILAYERS OF DIPALMITOYL3-SN-PHOSPHATIDYLCHOLINE. Biochemistry, 1975. 14(16): p. 36473652 . 
81. Seelig, J. and H.U. Gally, INVESTIGATION OF

PHOSPHATIDYLETHANOLAMINE BILAYERS BY DEUTERIUM AND P31 NUCLEAR MAGNETIC-RESONANCE. Biochemistry, 1976. 15(24): $\mathrm{p}$. 5199-5204.

82. Frey, P.A., S.A. Whitt, and J.B. Tobin, A LOW-BARRIER HYDROGENBOND IN THE CATALYTIC TRIAD OF SERINE PROTEASES. Science, 1994. 264(5167): p. 1927-1930.

83. Lee, C.W.B. and R.G. Griffin, TWO-DIMENSIONAL H-1/C-13

HETERONUCLEAR CHEMICAL-SHIFT CORRELATION

SPECTROSCOPY OF LIPID BILAYERS. Biophysical Journal, 1989.

55(2): p. 355-358.

84. Bjerrum, J.S., G.; Sillen, L.G. , ed. Stability Constants. Vol. 2. 1958, The Chemical Society: London.

85. Jeener, J., in Ampere Summer School. 1971: Basko Polje, Yugoslavia.

86. Aue, W.P., E. Bartholdi, and R.R. Ernst, 2-dimensional spectroscopy application to nuclear magnetic resonance. Journal of Chemical Physics, 1976. 64(5): p. 2229-2246.

87. Vega, S., T.W. Shattuck, and A. Pines, FOURIER-TRANSFORM DOUBLE-QUANTUM NMR IN SOLIDS. Physical Review Letters, 1976. 37(1): p. 43-46.

88. Hahn, E.L., Concepts of NMR in quantum optics. Concepts in Magnetic Resonance, 1997. 9(2): p. 69-81.

89. Pines, A., et al., NEW APPROACH TO HIGH-RESOLUTION PROTON NMR IN SOLIDS - DEUTERIUM SPIN DECOUPLING BY MULTIPLEQUANTUM TRANSITIONS. Physical Review Letters, 1976. 36(2): p. 110113.

90. Bodenhausen, G., R.L. Vold, and R.R. Vold, MULTIPLE QUANTUM SPINECHO SPECTROSCOPY. Journal of Magnetic Resonance, 1980. 37(1): p. 93-106.

91. Tang, J. and A. Pines, MULTIPLE QUANTUM NMR AND RELAXATION OF AN ORIENTED CH3 GROUP. Journal of Chemical Physics, 1980. 72(5): p. 3290-3297.

92. Pekar, J. and J.S. Leigh, DETECTION OF BIEXPONENTIAL RELAXATION IN NA-23 FACILITATED BY DOUBLE-QUANTUM FILTERING. Journal of Magnetic Resonance, 1986. 69(3): p. 582-584. 
93. Piantini, U., O.W. Sorensen, and R.R. Ernst, MULTIPLE QUANTUM FILTERS FOR ELUCIDATING NMR COUPLING NETWORKS. Journal of the American Chemical Society, 1982. 104(24): p. 6800-6801.

94. Bax, A., R. Freeman, and S.P. Kempsell, NATURAL ABUNDANCE C-13C-13 COUPLING OBSERVED VIA DOUBLE-QUANTUM COHERENCE. Journal of the American Chemical Society, 1980. 102(14): p. 4849-4851.

95. Filip, X., et al., New solid state-NMR techniques for investigating structure and dynamics in soft solids and biomaterials. Journal of Optoelectronics and Advanced Materials, 2008. 10(9): p. 2277-2281.

96. Saalwachter, K., Proton multiple-quantum NMR for the study of chain dynamics and structural constraints in polymeric soft materials. Progress in Nuclear Magnetic Resonance Spectroscopy, 2007. 51(1): p. 1-35.

97. Fechete, R., D.E. Demco, and B. Blumich, Enhanced sensitivity to residual dipolar couplings of elastomers by higher-order multiple-quantum NMR. Journal of Magnetic Resonance, 2004. 169(1): p. 19-26.

98. Meersmann, T., S.A. Smith, and G. Bodenhausen, Multiple-quantum filtered xenon-131 NMR as a surface probe. Physical Review Letters, 1998. 80(7): p. 1398-1401.

99. Frish, L., et al., The interaction of water molecules with purple membrane suspension using $\mathrm{H}-2$ double-quantum filter, $\mathrm{H}-1$ and $\mathrm{H}-2$ diffusion nuclear magnetic resonance. Biopolymers, 2004. 75(1): p. 46-59.

100. Vanderveen, J.R., M.A. Blackburn, and K.J. Ooms, (2)H double- and zeroquantum filtered NMR spectroscopy for probing the environments of water in Nafion. Canadian Journal of Chemistry-Revue Canadienne De Chimie, 2011. 89(9): p. 1095-1104.

101. Warren, W.S., et al., MR imaging contrast enhancement based on intermolecular zero quantum coherences. Science, 1998. 281(5374): p. 247-251.

102. Tsoref, L., et al., Slice-selective proton double quantum filtered MRI of joint connective tissues. Jmri-Journal of Magnetic Resonance Imaging, 2000. 11(3): p. 336-341.

103. Navon, G., et al., Study of order and dynamic processes in tendon by NMR and MRI. Journal of Magnetic Resonance Imaging, 2007. 25(2): p. 362-380.

104. Shinar, $\mathrm{H}$., et al., Mapping the fiber orientation in articular cartilage at rest and under pressure studied by $\mathrm{H}-2$ double quantum filtered MRI. Magnetic Resonance in Medicine, 2002. 48(2): p. 322-330. 
105. Sharf, Y., et al., Mapping strain exerted on blood vessel walls using deuterium double-quantum-filtered MRI. Proceedings of the National Academy of Sciences of the United States of America, 1998. 95(8): $p$. 4108-4112.

106. Doronin, S.I., et al., Multiple quantum NMR dynamics of spin-1/2 carrying molecules of a gas in nanopores. Journal of Chemical Physics, 2009. 131(10).

107. Chen, S., S.H. Cai, and Z. Chen, Intermolecular multiple-quantum coherences between spin 1/2 and quadrupolar nuclei in liquid nuclear magnetic resonance. Chemical Physics Letters, 2008. 458(4-6): p. 368372.

108. Perea, W., et al., H-2 double quantum filtered (DQF) NMR spectroscopy of the nucleus pulposus tissues of the intervertebral disc. Magnetic Resonance in Medicine, 2007. 57(6): p. 990-999.

109. Ooms, K.J., et al., Na-23 TQF NMR imaging for the study of spinal disc tissue. Journal of Magnetic Resonance, 2008. 195(1): p. 112-115.

110. Eliav, U. and G. Navon, Multiple quantum filtered NMR studies of the interaction between collagen and water in the tendon. Journal of the American Chemical Society, 2002. 124(12): p. 3125-3132.

111. Fechete, R., et al., Anisotropy of collagen fiber orientation in sheep tendon by $\mathrm{H}-1$ double-quantum-filtered NMR signals. Journal of Magnetic Resonance, 2003. 162(1): p. 166-175.

112. Sun, C. and G.S. Boutis, Investigation of the dynamical properties of water in elastin by deuterium Double Quantum Filtered NMR. Journal of Magnetic Resonance, 2010. 205(1): p. 86-92.

113. Sun, C., et al., NMR studies of localized water and protein backbone dynamics in mechanically strained elastin. The journal of physical chemistry. B, 2011. 115(47): p. 13935-42.

114. Richard R. Ernst, Geoffrey Bodenhausen, and A. Wokaun, Principles of nuclear magnetic resonance in one and two dimensions. The international series of monographs on chemistry, ed. R. Breslow, J. Halperin, and J.S. Rowlinson. 1987, New York: Oxford University Press. 610.

115. Slichter, C.P., Principles of Magnetic Resonance. 1963, New York: Harper and Row.

116. Wokaun, A. and R.R. Ernst, SELECTIVE DETECTION OF MULTIPLE QUANTUM TRANSITIONS IN NMR BY 2-DIMENSIONAL SPECTROSCOPY. Chemical Physics Letters, 1977. 52(3): p. 407-412. 
117. Eaton, J., Octave, URL:

http://www.gnu.org/software/octave/download.html.

118. Hoeve, C.A.J. and P.J. Flory, ELASTIC PROPERTIES OF ELASTIN. Biopolymers, 1974. 13(4): p. 677-686.

119. Urry, D.W., et al., Elastin: a representative ideal protein elastomer. Philosophical Transactions of the Royal Society of London Series BBiological Sciences, 2002. 357(1418): p. 169-184.

120. Urry, D.W., Venkatachalam, C.M., Long, M.M., Prasad, K.U., Dynamic $\beta$ spirals and a librational entropy mechanism of elasticity, in Conformation in biology, R. Srinivasan, Sarma, R.H., Editor. 1982, Adenine Press:

Guilderland, NY. p. 11-27.

121. Weis-Fogh, T. and S.O. Andersen, NEW MOLECULAR MODEL FOR LONG-RANGE ELASTICITY OF ELASTIN. Nature, 1970. 227(5259): p. 718-\&.

122. Gosline, J.M., HYDROPHOBIC INTERACTION AND A MODEL FOR ELASTICITY OF ELASTIN. Biopolymers, 1978. 17(3): p. 677-695.

123. Gosline, J.M., F.F. Yew, and T. Weisfogh, REVERSIBLE STRUCTURALCHANGES IN A HYDROPHOBIC PROTEIN, ELASTIN, AS INDICATED BY FLUORESCENCE PROBE ANALYSIS. Biopolymers, 1975. 14(9): p. 1811-1826.

124. Li, B., et al., Hydrophobic hydration is an important source of elasticity in elastin-based biopolymers. Journal of the American Chemical Society, 2001. 123(48): p. 11991-11998.

125. Dill, K.A., DOMINANT FORCES IN PROTEIN FOLDING. Biochemistry, 1990. 29(31): p. 7133-7155.

126. Li, B., D.O.V. Alonso, and V. Daggett, The molecular basis for the inverse temperature transition of elastin. Journal of Molecular Biology, 2001. 305(3): p. 581-592.

127. McQuarrie, D.A., Simon, J.D., Physical Chemistry: a Molecular Approach. 1997, Sausalito, CA: University Science Books.

128. Palmer, A.G., NMR probes of molecular dynamics: Overview and comparison with other techniques. Annual Review of Biophysics and Biomolecular Structure, 2001. 30: p. 129-155.

129. Yang, D.W. and L.E. Kay, Contributions to conformational entropy arising from bond vector fluctuations measured from NMR-derived order 
parameters: Application to protein folding. Journal of Molecular Biology, 1996. 263(2): p. 369-382.

130. Yang, D.W., et al., Contributions to protein entropy and heat capacity from bond vector motions measured by NMR spin relaxation. Journal of Molecular Biology, 1997. 272(5): p. 790-804.

131. Li, Z.G., S. Raychaudhuri, and A.J. Wand, Insights into the local residual entropy of proteins provided by NMR relaxation. Protein Science, 1996. 5(12): p. 2647-2650.

132. Chandler, D., Interfaces and the driving force of hydrophobic assembly. Nature, 2005. 437(7059): p. 640-647.

133. Wittebort, R.J., et al., OBSERVATION OF MOLECULARREORIENTATION IN ICE BY PROTON AND DEUTERIUM MAGNETICRESONANCE. Journal of the American Chemical Society, 1988. 110(17): p. 5668-5671.

134. McBriert.Vj, USE OF ROTATION OPERATORS IN GENERAL DESCRIPTION OF POLYMER PROPERTIES. Journal of Chemical Physics, 1974. 61(3): p. 872-882.

135. Brainard, J.R. and A. Szabo, THEORY FOR NUCLEAR MAGNETICRELAXATION OF PROBES IN ANISOTROPIC SYSTEMS APPLICATION TO CHOLESTEROL IN PHOSPHOLIPID-VESICLES. Biochemistry, 1981. 20(16): p. 4618-4628.

136. Lipari, G. and A. Szabo, EFFECT OF LIBRATIONAL MOTION ON FLUORESCENCE DEPOLARIZATION AND NUCLEAR MAGNETICRESONANCE RELAXATION IN MACROMOLECULES AND MEMBRANES. Biophysical Journal, 1980. 30(3): p. 489-506.

137. Hahn, E.L., Spin Echoes. Physical Review, 1950. 80(4): p. 580-594.

138. Abragam, A., The Principles of Nuclear Magnetism. 1961, Oxford, England: Oxford University Press.

139. Lillie, M.A., G.W.G. Chalmers, and J.M. Gosline, Elastin dehydration through the liquid and the vapor phase: A comparison of osmotic stress models. Biopolymers, 1996. 39(5): p. 627-639.

140. Zhang, Q.W., et al., Double and triple resonance circuits for highfrequency probes. Journal of Magnetic Resonance, 1998. 132(1): p. 167171. 
141. Connelly, G.P., et al., ISOTOPE EFFECTS IN PEPTIDE GROUP HYDROGEN-EXCHANGE. Proteins-Structure Function and Genetics, 1993. 17(1): p. 87-92.

142. Farrar, T.C. and J.A. Ropp, Experimental NMR relaxation studies of simple hydrogen-bonded liquids. Journal of Molecular Liquids, 2002. 98-9: p. 103-127.

143. Ropp, J., et al., Rotational motion in liquid water is anisotropic: A nuclear magnetic resonance and molecular dynamics simulation study. Journal of the American Chemical Society, 2001. 123(33): p. 8047-8052.

144. Gillen, K.T., D.C. Douglass, and J.R. Hoch, SELF-DIFFUSION IN LIQUID WATER TO-31 DEGREESC. Journal of Chemical Physics, 1972. 57(12): p. 5117-\&.

145. Wasano, $\mathrm{K}$. and T. Yamamoto, TRIDIMENSIONAL ARCHITECTURE OF ELASTIC TISSUE IN THE RAT AORTA AND FEMORAL-ARTERY - A SCANNING ELECTRON-MICROSCOPE STUDY. Journal of Electron Microscopy, 1983. 32(1): p. 33-44.

146. Toonkool, P., et al., Hydrophobic domains of human tropoelastin interact in a context-dependent manner. Journal of Biological Chemistry, 2001. 276(48): p. 44575-44580.

147. Usha, M.G., W.L. Peticolas, and R.J. Wittebort, DEUTERIUM QUADRUPOLE COUPLING IN N-ACETYLGLYCINE AND LIBRATIONAL DYNAMICS IN SOLID POLY(GAMMA-BENZYL-L-GLUTAMATE). Biochemistry, 1991. 30(16): p. 3955-3962.

148. Wittebort, R.J. and A. Szabo, THEORY OF NMR RELAXATION IN MACROMOLECULES - RESTRICTED DIFFUSION AND JUMP MODELS FOR MULTIPLE INTERNAL ROTATIONS IN AMINO-ACID SIDECHAINS. Journal of Chemical Physics, 1978. 69(4): p. 1722-1736.

149. Walther, K.A., et al., Signatures of hydrophobic collapse in extended proteins captured with force spectroscopy. Proceedings of the National Academy of Sciences of the United States of America, 2007. 104(19): $p$. 7916-7921.

150. Deloche, B. and E.T. Samulski, SHORT-RANGE NEMATIC-LIKE ORIENTATIONAL ORDER IN STRAINED ELASTOMERS - A DEUTERIUM MAGNETIC-RESONANCE STUDY. Macromolecules, 1981. 14(3): p. $575-581$.

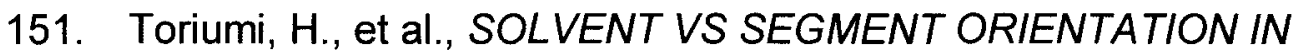
STRAINED SWOLLEN ELASTOMERIC NETWORKS. Macromolecules, 1985. 18(2): p. 304-305. 
152. Deloche, B. and E.T. Samulski, RUBBER ELASTICITY - A PHENOMENOLOGICAL APPROACH INCLUDING ORIENTATIONAL CORRELATIONS. Macromolecules, 1988. 21(10): p. 3107-3111.

153. Li, I.T.S. and G.C. Walker, Signature of hydrophobic hydration in a single polymer. Proceedings of the National Academy of Sciences of the United States of America, 2011. 108(40): p. 16527-16532. 


\section{APPENDIX A \\ ${ }^{17}$ O MULTIPLE QUANTUM NMR}

${ }^{17} \mathrm{O}$ is potentially useful for characterization of weakly ordered water since it is not exchangeable with sites on the polymer, i.e. the signal originates only from the solvent. Moreover, the larger quadrupolar coupling implies more accurate determination of small order parameters, as in case of weakly ordered water. Implementation of $\mathrm{MQ}$ filtering approach to ${ }^{17} \mathrm{O}$ requires more sophisticated than for ${ }^{2} \mathrm{H}$ schemes for studying particular coherences. The pulse sequence and phase cycle devised in Chapter 3 for spins with $I=1$, require modification for $\mathrm{I}=5 / 2\left({ }^{17} \mathrm{O}\right)$ because the number of transitions is larger and additional actions are required to eliminate multiple quantum interference effects.

\section{A.1. Transition frequencies and operators}

\section{Transition frequencies}

The transition frequencies at which different $M Q$ coherences are evolving are calculated from the general formula

$$
\omega^{m m^{\prime}}=E^{m}-E^{m} / \hbar
$$

for transition between states $m$ and $m^{\prime}$. Energy levels are

$$
E^{m}=E_{o s}^{m}+E_{q}^{m},
$$


where $E_{o s}^{m}$ and $E_{q}^{m}$ are the diagonal matrix elements

$$
\begin{aligned}
& E_{o s}^{m}=\left\langle m\left|H_{o s}\right| m\right\rangle, \\
& E_{q}^{m}=\left\langle m\left|H_{q}\right| m\right\rangle .
\end{aligned}
$$

Hamiltonians $H_{o s}$ and $H_{q}$ are given in chapter 3 (page 38). For $I=5 / 2$, possible spin states are $m=5 / 2,3 / 2,1 / 2,-1 / 2,-3 / 2,-5 / 2$ and the quadrupolar coupling constant is $\omega_{q}=3 e q Q^{2} / 40 \hbar$. To $1^{\text {st }}$ order, only the part of $H_{q}$ that commutes with the Zeeman Hamiltonian, $\mathrm{H}^{0}$, is required,

$$
H_{q}=\omega_{q}\left(I_{z}^{2}-35 / 12\right) \text {. }
$$

In total, there are 15 possible transitions between 6 states as follows: 5 single quantum transitions (1Q), 4 double quantum transitions $(2 Q), 3$ triple quantum transitions (3Q), 2 quadruple quantum transitions (4Q) and one pentuple quantum transition (5Q). The $1 \mathrm{Q}$ frequencies are

$$
\begin{aligned}
& \omega_{12}=E^{-5 / 2}-E^{-3 / 2}=\Delta \omega_{o s}+4 \omega_{q}, \omega_{23}=E^{-3 / 2}-E^{-1 / 2}=\Delta \omega_{o s}+2 \omega_{q}, \\
& \omega_{56}=E^{3 / 2}-E^{5 / 2}=\Delta \omega_{o s}-4 \omega_{q}, \omega_{45}=E^{1 / 2}-E^{3 / 2}=\Delta \omega_{o s}-2 \omega_{q} \text { and } \\
& \omega_{34}=E^{-5 / 2}-E^{-3 / 2}=\Delta \omega_{o s},
\end{aligned}
$$

with satellite splitting of $0,4 \omega_{q}$ and $8 \omega_{q} .2 Q$ frequencies are

$$
\begin{aligned}
& \omega_{13}=E^{-5 / 2}-E^{-1 / 2}=2 \Delta \omega_{o s}+6 \omega_{q}, \omega_{24}=E^{-3 / 2}-E^{1 / 2}=2 \Delta \omega_{o s}+2 \omega_{q}, \\
& \omega_{35}=E^{-1 / 2}-E^{3 / 2}=2 \Delta \omega_{o s}-6 \omega_{q} \text { and } \omega_{46}=E^{1 / 2}-E^{5 / 2}=2 \Delta \omega_{o s}-2 \omega_{q},
\end{aligned}
$$

with satellite splitting $4 \omega_{\mathrm{q}}$ and $12 \omega_{\mathrm{q}}$. Three $3 Q$ frequencies with satellite splitting 0 and $12 \omega_{\mathrm{q}}$ are 
$\omega_{14}=E^{-5 / 2}-E^{1 / 2}=3 \Delta \omega_{o s}+6 \omega_{q}, \omega_{25}=E^{-3 / 2}-E^{3 / 2}=3 \Delta \omega_{o s}$ and

$\omega_{36}=E^{-1 / 2}-E^{5 / 2}=3 \Delta \omega_{o s}-6 \omega_{q}$.

The two $4 Q$ transitions are

$\omega_{15}=E^{-5 / 2}-E^{3 / 2}=4 \Delta \omega_{o s}+4 \omega_{q}$ and $\omega_{26}=E^{-3 / 2}-E^{5 / 2}=4 \Delta \omega_{o s}-4 \omega_{q}$,

and a single $5 Q$ transition frequency is

$\omega_{16}=E^{-5 / 2}-E^{5 / 2}=5 \Delta \omega_{o s}$.

From above, it is clear that only two MQ coherencies are independent of the quadrupolar coupling. These are the $5 \mathrm{Q}$ and the central $3 \mathrm{Q}$ transition, which evolve at $5 \Delta \omega_{\mathrm{os}}$ and $3 \Delta \omega_{\mathrm{os}}$, respectively. Detection of these transitions is favorable since there is no quadrupolar broadening due to their independence on $\omega_{\mathrm{q}}$.

\section{Basis operators}

For ${ }^{17} \mathrm{O}, \mathrm{I}=5 / 2$, there are $2 \mathrm{I}+1=6$ spin states and the density operator has $6 \times 6=36$ elements. Therefore, to represent the density operator as a linear combination of basis operators, the basis set has 36 operators. We use, as in the $I$ = 1 case, a set obtained from single transition operators, $l_{k}^{r s}$. In addition to the identity operator, these are the coherences, $l_{x}^{r s}$ and $l_{y}^{\text {rs }}$ (for the 15 rs values), and

5 operators $\mathrm{I}_{z}^{\mathrm{r}, \mathrm{r}+1}$ that are orthogonalized by the Gram-Schmidt procedure as follows:

$\mathrm{O}_{z}^{12}=\mathrm{I}_{\mathrm{z}}^{12}, \quad \mathrm{O}_{\mathrm{z}}^{23}=\mathrm{I}_{\mathrm{z}}^{23}-\frac{\operatorname{Tr}\left(\mathrm{O}_{\mathrm{z}}^{12} \mathrm{I}_{\mathrm{z}}^{23}\right)}{\operatorname{Tr}\left(\mathrm{O}_{\mathrm{z}}^{12} \mathrm{O}_{\mathrm{z}}^{12}\right)} \mathrm{O}_{\mathrm{z}}^{12}$, 


$$
\begin{aligned}
& \mathrm{O}_{z}^{34}=\mathrm{l}_{z}^{34}-\frac{\operatorname{Tr}\left(\mathrm{O}_{z}^{23} \mathrm{l}_{z}^{34}\right)}{\operatorname{Tr}\left(\mathrm{O}_{\mathrm{z}}^{23} \mathrm{O}_{\mathrm{z}}^{23}\right)} \mathrm{O}_{\mathrm{z}}^{23}-\frac{\operatorname{Tr}\left(\mathrm{O}_{\mathrm{z}}^{12} \mathrm{l}_{\mathrm{z}}^{34}\right)}{\operatorname{Tr}\left(\mathrm{O}_{\mathrm{z}}^{12} \mathrm{O}_{\mathrm{z}}^{12}\right)} \mathrm{O}_{\mathrm{z}}^{12} \\
& \mathrm{O}_{z}^{45}=\mathrm{l}_{\mathrm{z}}^{45}-\frac{\operatorname{Tr}\left(\mathrm{O}_{z}^{34} \mathrm{l}_{\mathrm{z}}^{45}\right)}{\operatorname{Tr}\left(\mathrm{O}_{\mathrm{z}}^{34} \mathrm{O}_{\mathrm{z}}^{34}\right)} \mathrm{O}_{\mathrm{z}}^{34}-\frac{\operatorname{Tr}\left(\mathrm{O}_{z}^{23} \mathrm{l}_{z}^{45}\right)}{\operatorname{Tr}\left(\mathrm{O}_{\mathrm{z}}^{23} \mathrm{O}_{z}^{23}\right)} \mathrm{O}_{\mathrm{z}}^{23}-\frac{\operatorname{Tr}\left(\mathrm{O}_{\mathrm{z}}^{12} \mathrm{I}_{\mathrm{z}}^{45}\right)}{\operatorname{Tr}\left(\mathrm{O}_{\mathrm{z}}^{12} \mathrm{O}_{\mathrm{z}}^{12}\right)} \mathrm{O}_{\mathrm{z}}^{12} \text {, }
\end{aligned}
$$



Since $I_{x}^{r s}$ and $I_{y}^{r s}$ are normalized to $1 / 2, O_{z}^{r, r+1}$ are normalized also to $1 / 2$. These operators are given in Table A.1.

Table A.1. Matrix representations of orthogonalized operators for $I=5 / 2$.

$$
\begin{aligned}
& O_{z}^{12}=\left(\begin{array}{cccccc}
a & 0 & 0 & 0 & 0 & 0 \\
0 & -a & 0 & 0 & 0 & 0 \\
0 & 0 & 0 & 0 & 0 & 0 \\
0 & 0 & 0 & 0 & 0 & 0 \\
0 & 0 & 0 & 0 & 0 & 0 \\
0 & 0 & 0 & 0 & 0 & 0
\end{array}\right), a=f(1) \quad O_{z}^{23}=\left(\begin{array}{ccccccc}
b & 0 & 0 & 0 & 0 & 0 \\
0 & b & 0 & 0 & 0 & 0 \\
0 & 0 & -2 b & 0 & 0 & 0 \\
0 & 0 & 0 & 0 & 0 & 0 \\
0 & 0 & 0 & 0 & 0 & 0 \\
0 & 0 & 0 & 0 & 0 & 0
\end{array}\right), b=f(2) \\
& O_{z}^{34}=\left(\begin{array}{cccccc}
c & 0 & 0 & 0 & 0 & 0 \\
0 & c & 0 & 0 & 0 & 0 \\
0 & 0 & c & 0 & 0 & 0 \\
0 & 0 & 0 & -3 c & 0 & 0 \\
0 & 0 & 0 & 0 & 0 & 0 \\
0 & 0 & 0 & 0 & 0 & 0
\end{array}\right), \mathrm{c}=f(3) \quad O_{z}^{45}=\left(\begin{array}{cccccc}
d & 0 & 0 & 0 & 0 & 0 \\
0 & d & 0 & 0 & 0 & 0 \\
0 & 0 & d & 0 & 0 & 0 \\
0 & 0 & 0 & d & 0 & 0 \\
0 & 0 & 0 & 0 & -4 d & 0 \\
0 & 0 & 0 & 0 & 0 & 0
\end{array}\right), d=f(4) \\
& O_{z}^{56}=\left(\begin{array}{cccccc}
e & 0 & 0 & 0 & 0 & 0 \\
0 & e & 0 & 0 & 0 & 0 \\
0 & 0 & e & 0 & 0 & 0 \\
0 & 0 & 0 & e & 0 & 0 \\
0 & 0 & 0 & 0 & e & 0 \\
0 & 0 & 0 & 0 & 0 & -5 e
\end{array}\right), \mathrm{e}=f(5)
\end{aligned}
$$




\section{A.2. Scripts}

Octave density matrix scripts allow numerical simulation of the evolution of the spin system during times and under the interactions determined by the pulse sequence. In addition, phases of the pulses are set separately and allow developing of a phase cycle. In this way, each coherence can be plotted as a function of preparation time and these graphs are used to determine optimal preparation times (maximum signal) for a given quadrupolar coupling. The "\#" character indicates comment lines.

The script "mq_017" simulates pulse sequence and phase cycle. An illustration of the settings used in the script is shown in Figure A.1. Main script "mq_017" requires additional scripts for creation of relevant operators ("ops6levels"), simulation of RF pulses ("evol_ThPh") and evolution periods ("evol_Q_CS"). At any time current density operator can be decomposed into linear combination of basis operators using script "decomp".

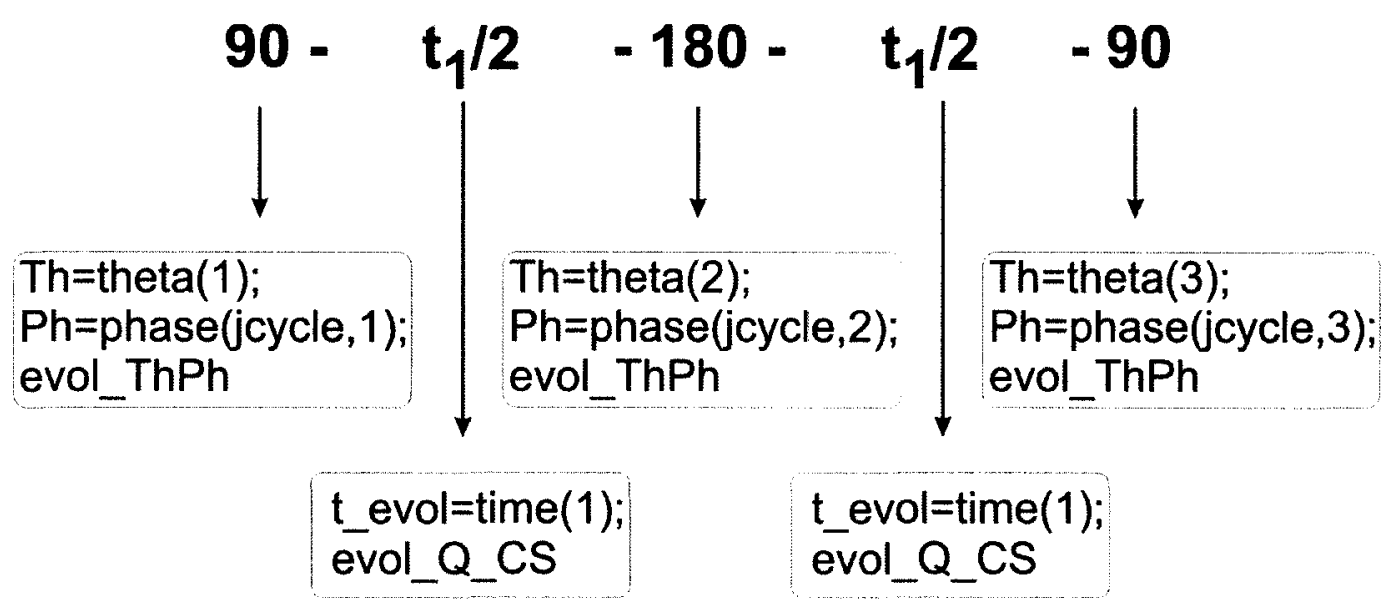

Figure A.1. Settings for the pulse sequence script, "mq_017". 


\section{mq_017}

clear
$l=5 / 2$

$\mathrm{I}=5 / 2$

\# initiate the sequence by obtaining the necessary spin operators and by

\# setting values of the quadrupolar coupling, vq, and offset (vos)

\# frequencies (in $\mathrm{Hz}$ )

\# delays

\# 2*time(1) = evolution time in preparation and conversion, time(2)=echo delay

\# for refocusing $2 \mathrm{Q}$

\# evolution, time(3)=2Q evolution following refocusing.

\# Total time between $1 Q$ to $2 Q$ preparation and $2 Q$ to $1 Q$ conversion periods is \# 2*time(2)+time(3)

time $=[.125, .10,0]$

\# pulses widths (deg) for all pulses

\# in the order that they appear in the sequence

theta $=[90,180,90,180,90,180]$

\# pulse phases phase(jcycle,kpulse) with jcycle=cycle number

\# and kpulse=pulse number

phase $=[0,90,90,0,90,0$;

$0,180,90,0,90,0$;

$0,0,90,90,0,0$

$0,0,90,90,90,0$

$90,0,180,90,0,0$;

$0,0,90,0,90,0$;

$0,0,0,270,0,0$

$0,0,0,315,0,0$;

$180,180,180,0,0,0$;

$180,180,180,45,0,0$;

$180,180,180,90,0,0$;

$180,180,180,135,0,0$;

$180,180,180,180,0,0$;

$180,180,180,225,0,0$;

$180,180,180,270,0,0$;

$180,180,180,315,0,0]$

\# set vos and vq

$v q=1$

vos $=0$

\# Initialize sequence by creating relevant operators

ops6levels;

for jcycle=1:1:2;

\# set the initial density operator to lz

rho=lz; 
\# step 1-5 of sequence, MQ preparation

Th=theta(1);

$\mathrm{Ph}=$ phase(jcycle, 1);

evol_ThPh;

'coherences after initial 90'

decomp

t_evol=time(1);

evol_Q_CS;

Th=theta(2);

$\mathrm{Ph}=\mathrm{phase}(\mathrm{jcycle}, 2)$;

evol_ThPh;

t_evol=time(1);

evol_Q_CS;

'coherences prior to 2 nd 90 in preparation'

decomp

Th=theta(3);

Ph=phase(jcycle,3);

evol_ThPh;

'coherences after $2 \mathrm{Q}$ preparation is complete'

decomp

\# steps 6-8, DQ echo and evolution

$\mathrm{t}$ evol=time(2);

evol_Q_CS;

Th=theta(4);

Ph=phase (jcycle,4);

evol_ThPh;

t_evol=time(2);

evol_Q_CS;

'mq refocusing complete'

decomp

\# MQ evolution following refocusing

t_evol=time(3);

evol_Q_CS;

'evolution complete, before conversion pulse'

decomp

\# step 9 detection

Th=theta(5);

$\mathrm{Ph}=$ phase (jcycle,5);

evol_ThPh;

'cohērences after conversion pulse'

decomp

endfor 
The script "ops6levels" creates basis operators for $I=5 / 2$.

\section{ops6levels}

\# Prepares the $306 \times 6$ matrix representations of spin $5 / 2$ single transition \#coherences, $I X(r, s)=I(1, r, s,:,:)$ and $\operatorname{ly}(2, r, s,:,:) ; r=1->5, s=r+1->6$, \# 5 orthogonal polarization operators, $I(3, r, s, .,:) ; r=1->5, s=r+1$, \#lx, ly, Iz.

\#and the raising and lowering operators, $1+=1 \mathrm{l} ; \mathrm{I}-=\mathrm{Im}$ dum=zeros(6); for $r=1: 1: 5$; for $s=r+1: 1: 6$

$$
\begin{aligned}
& \mathrm{I}(1, \mathrm{r}, \mathbf{s}, .,:)=\operatorname{dum}(:,:) ; \\
& I(1, r, s, r, s)=1 / 2 \text {; } \\
& \mathrm{I}(1, \mathrm{r}, \mathrm{s}, \mathrm{s}, \mathrm{r})=1 / 2 \text {; } \\
& \mathrm{I}(2, \mathrm{r}, \mathrm{s}, .,:)=\operatorname{dum}(:,:) \text {; } \\
& \mathrm{I}(2, \mathrm{r}, \mathrm{s}, \mathrm{r}, \mathrm{s})=-\mathrm{i} / 2 \text {; } \\
& \mathrm{I}(2, \mathrm{r}, \mathrm{s}, \mathrm{s}, \mathrm{r})=\mathrm{i} / 2 \text {; }
\end{aligned}
$$

endfor;

endfor;

\# check coherences

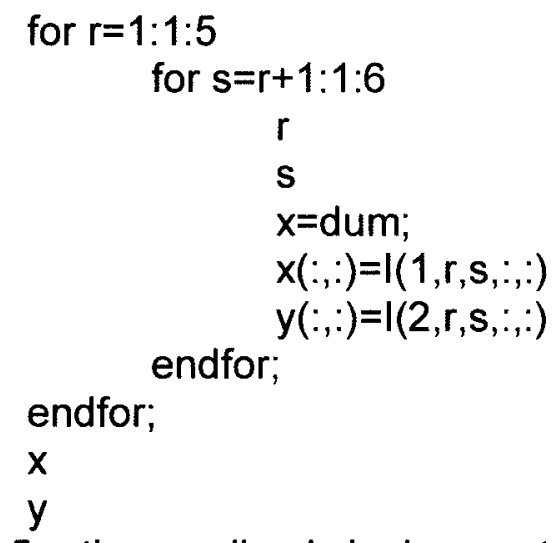

\# create the 5 orthogonalized single quantum polarizations,

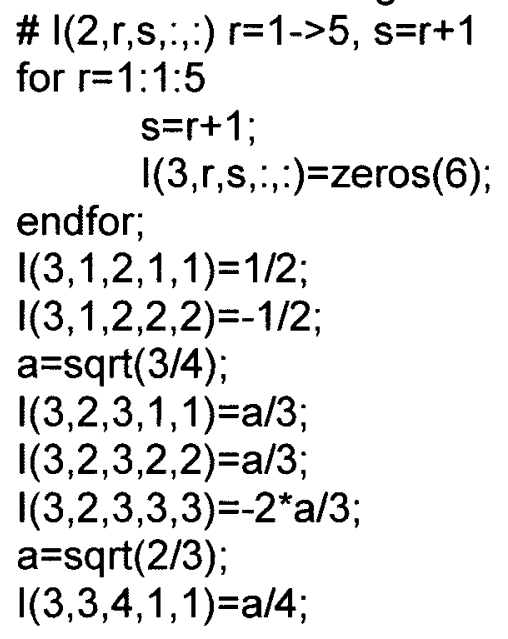







$n=1$;

while $(n<2 * j+1)$

$\operatorname{Ix}(n, n+1)=0.5^{*} \operatorname{sqrt}\left(n^{*}\left(2^{*} j+1-n\right)\right)$;

$\operatorname{lx}(n+1, n)=0.5^{\star} \operatorname{sqrt}\left(n^{\star}\left(2^{\star} j+1-n\right)\right)$;

$\mathrm{n++}$;

endwhile

Ix

\# matrix for the $y$-component of angular momentum

\# $\mid z$ and $\mid x$ are defined, so we just use commutator [ $|z| x]=i l y$ to find $l y$

$\mid y=(1 / i)^{\star}\left(\left|z^{*}\right| x-\left|x^{\star}\right| z\right)$;

$c k=i^{*}\left(l y^{\star}|x-| x^{\star} \mid y\right)$

$|\mathrm{p}=| \mathrm{x}+\mathrm{i}^{*} \mid \mathrm{y}$

The script "evol_ThPh" calculates the effect of an RF pulse with flip angle Th and

phase $\mathrm{Ph}$ on the current density operator, rho.

evol_ThPh

\# evolution of the current density operator, rho, subject to a hard RF

\# pulse with flip angle Th (deg) and phase $\mathrm{Ph}(\mathrm{deg})$

phir=pi*Ph/180;

thetar=pi* Th/180;

$o p=\left(\cos (p h i r)^{\star} \mid x\right)+\left(\sin (p h i r)^{\star} l y\right)$;

prop $=\operatorname{expm}\left(-i^{\star}(\text { thetar })^{\star} o p\right)$;

r=prop ${ }^{\prime *}$ rho*prop;

rho $=r$

The script "evol_Q_CS" calculates the effect of evolution under quadrupolar coupling and chemical shift on the current density operator, rho.

\section{evol_QCS}

$w q=2^{*} p i^{*} v q$

wos $=2^{*} \mathrm{pi}^{*}$ vos;

$\mathrm{Id}=\left(\left(\mathrm{sp}^{*}(\mathrm{sp}+1)\right) / 3\right)^{\star} \mathrm{eye}\left(2^{\star} \mathrm{sp}+1\right)$;

$\mathrm{Hq}=\mathrm{wq}^{*}((\mathrm{Iz} \mid \mathrm{z})-\mathrm{Id})$;

Hcs=wos*lz;

dum $=i^{*}(\mathrm{Hcs}+\mathrm{Hq})^{\star}$ t_evol;

prop=expm(-dum);

r=prop'*tho*prop;

rho=r

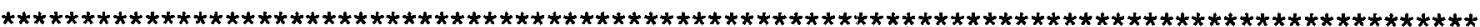


The script "decomp" takes the current density operator, rho, and decomposes it into a linear combination of basis operators.

\section{decomp}

\# decompose the density operator, rho, into single transition operators

\# and store in the complex vector st(q). For elements $q=1-->15$, real(st) and

\# imag(st) are Ix(rs), ly(rs), respectively, in the order $(r, s)=(1,2),(1,3), \ldots . .(1,6)$,

$\#(2,3) \ldots(5,6)$. Orthogonalized $I z(r, s)$ coefficients (real) are $q=16->20$ in the order $\#(r, s)=(1,2),(2,3) \ldots \ldots .(5,6)$. Finally, coefficients for $I x$ and ly are real(st(21) and \# imag(st(21)), i.e., st(21)=trace $\left(1+^{*} r h o\right)$, and $I z=s(22)$.

$q=0$;

for $r=1: 1: 5$;

for $s=r+1: 1: 6$

$q=q+1$

dum $=2^{*}$ real $($ rho $(r, s))$;

st $(q)=d u m$;

dum $=-2^{*}$ imag $($ rho $(r, s))$;

endfor; $s t(q)=s t(q)+i^{*} d u m ;$

endfor;

I=15;

for $r=1: 1: 5$;

$s=r+1$;

$\mathrm{I}=\mathrm{I}+1$;

dum $=0$;

for $\mathrm{jk}=1,6$;



endfor;

endfor

st $(I)=d u m$;

st(21)=trace $\left(\right.$ Ip $^{\star}$ rho);

st(22)=trace $\left(I^{*}\right.$ rho);

v1q=[st(1),st(6),st(10),st(13),st(15)]

$v 2 q=[s t(2), s t(7), s t(11), s t(14)]$

$v 3 q=[\operatorname{st}(3), s t(8), s t(12)]$

$v 4 q=[s t(4), s t(9)]$

$v 5 q=[\operatorname{st}(5)]$ 


\section{APPENDIX B}

\section{${ }^{2} \mathrm{H}$ SCRIPT}

Operators for $\mathrm{I}=1$ are given in chapter 3 (pages $41-42$ ). All scripts in appendix $A$ can be used for $I=1\left({ }^{2} H\right)$ simulations by substituting "ops6levels" with the following script, "ops3levels", that creates basis operators for $\mathrm{I}=1$.

\section{ops3levels}

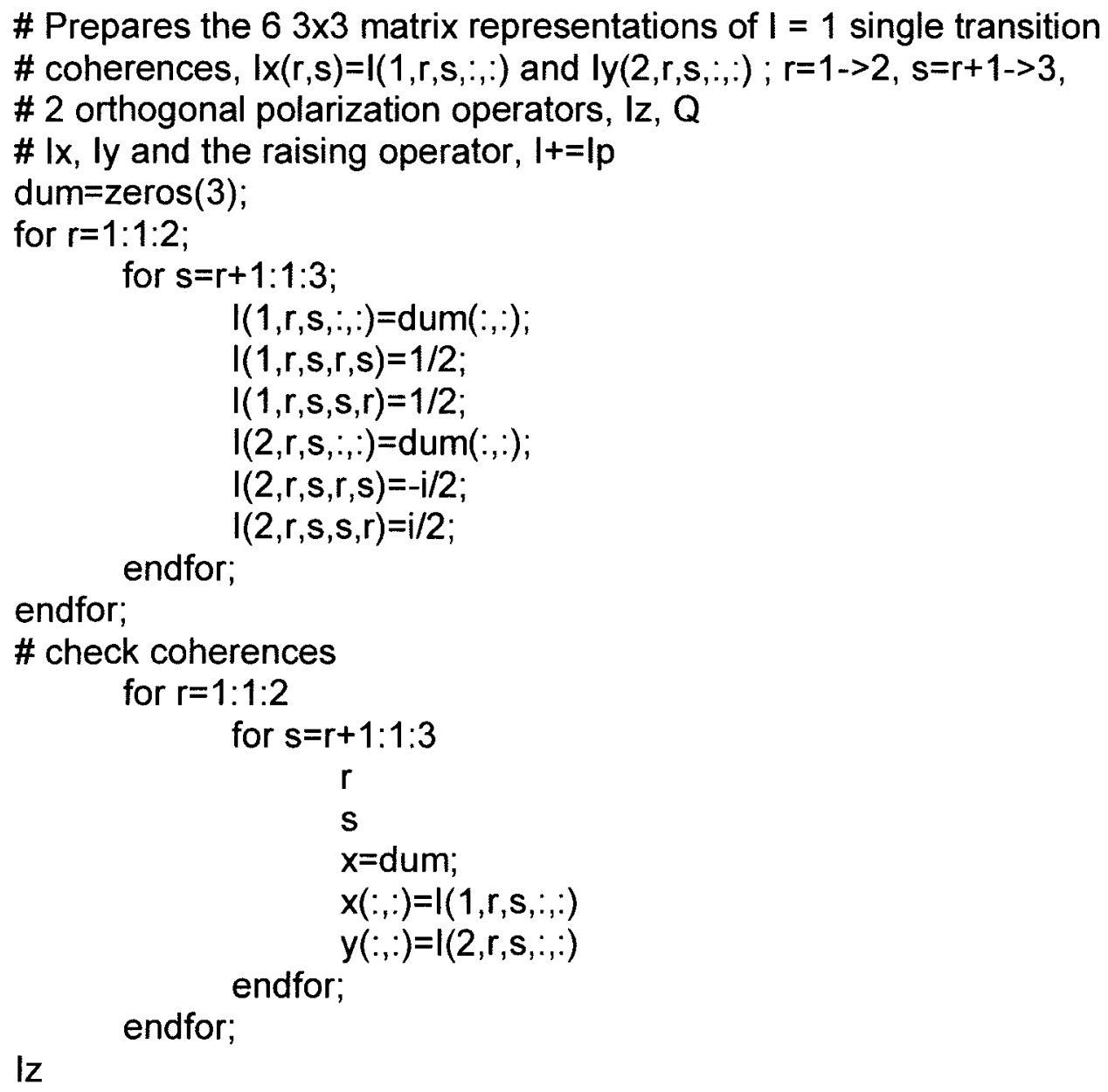




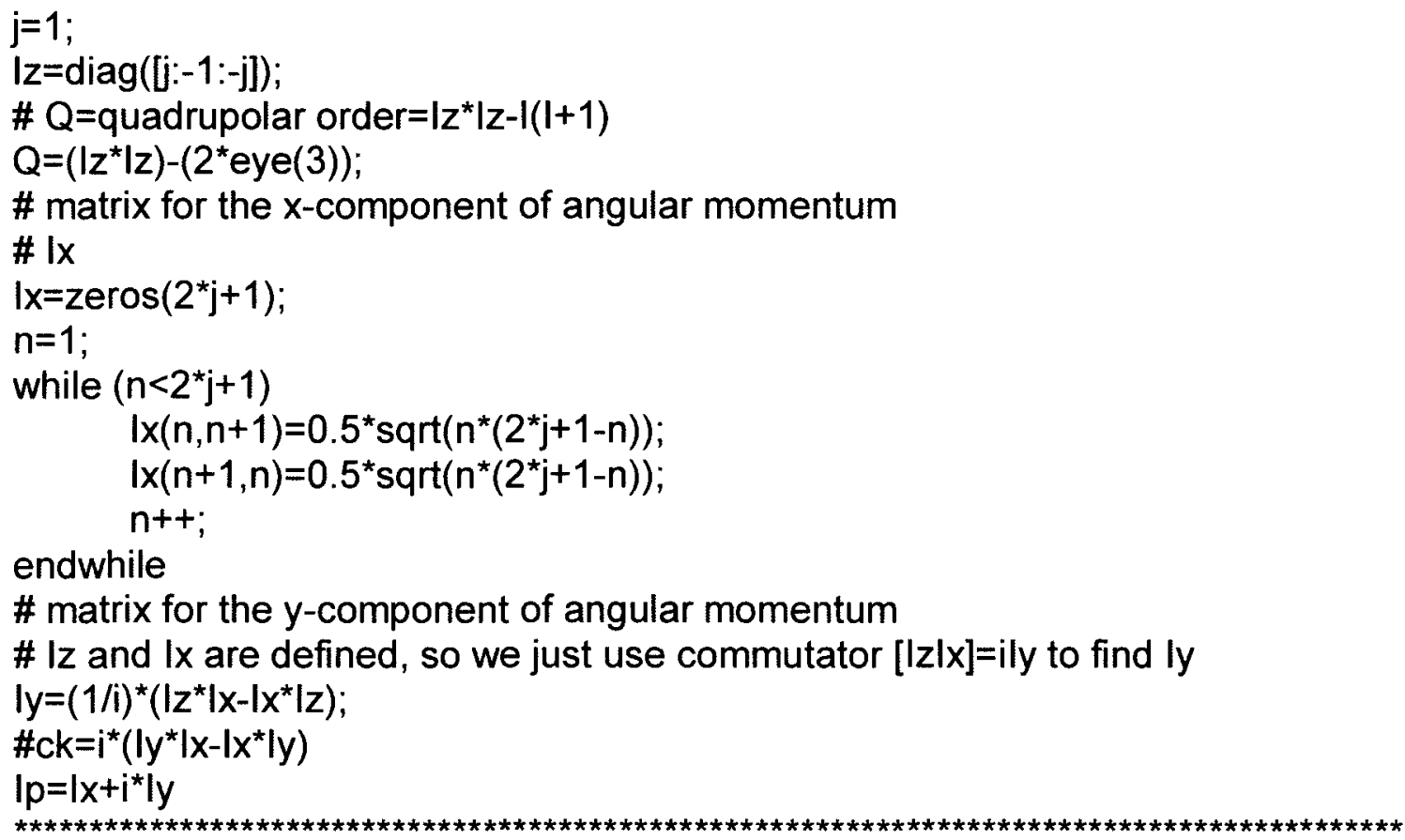




\section{APPENDIX C}

\section{${ }^{2} \mathrm{H}$ SPIN RELAXATION RATES}

Deuterium, ${ }^{2} \mathrm{H}$, is a quadrupolar nucleus and the dominant mechanism of spin relaxation is due to reorientation of the nuclear electric quadrupole moment relative to the applied field. For spin 1 nuclei, the rates are [138]

$$
R_{1}=\frac{1}{T_{1}}=C\left(1+\frac{\eta^{2}}{3}\right)\left(J\left(\omega_{0}\right)+4 J\left(2 \omega_{0}\right)\right)
$$

and

$$
R_{2}=\frac{1}{T_{2}}=\frac{1}{6} C\left(1+\frac{\eta^{2}}{3}\right)\left(9 J(0)+15 J\left(\omega_{0}\right)+6 J\left(2 \omega_{0}\right)\right)
$$

with

$$
C=\frac{3}{10} \pi^{2}\left(\frac{e^{2} q_{z z} Q}{h}\right)^{2}=\frac{3}{10} \pi^{2} v_{q}^{2}
$$

For diffusional motion in isotropic fluids,

$$
J\left(\omega_{0}\right)=\frac{T_{c}}{1+\left(\omega_{0} T_{c}\right)^{2}}
$$

The function $\mathrm{J}\left(\omega_{0}\right)$ is the spectral density (Fourier transform of the autocorrelation function) of the fluctuating field caused by diffusive reorientation. Relaxation is efficient when molecular motions, characterized by the correlation 
time $T_{c}$, have the same time scale as the reciprocal magnetic resonance frequency, $T_{c} \sim \omega_{0}$. In the above $\eta=\left(q_{y y}-q_{x x}\right) / q_{z z}$ is the asymmetry parameter of the quadrupolar coupling with $\left|q_{x x}\right| \leq\left|q_{y y}\right| \leq\left|q_{z z}\right|, v_{q}$ is the quadrupolar coupling constant in $\mathrm{Hz}, \mathrm{e}$ is the electron charge, $\mathrm{Q}$ is the nuclear quadrupolar moment, and $\mathrm{eq}_{\mathrm{zz}}$ is the principal z-component of the electric field gradient tensor.

Graphical representations of these equations are shown in Figure C.1. Short correlation times correspond to fast motion whereas slow molecular motion, as in viscous liquids, implies long correlation times. When correlation times are short (known as the extreme narrowing limit), $\omega_{0} T_{c} \ll 1$, as $T_{1}$ and $T_{2}$ are equal. $T_{1}$ reaches a minimum at $T_{c} \approx 1 / \omega_{0}$, and $T_{1}$ and $T_{2}$ diverge in the slow motion or long correlation time limit wherein $T_{1}$ increases and $T_{2}$ decreases.

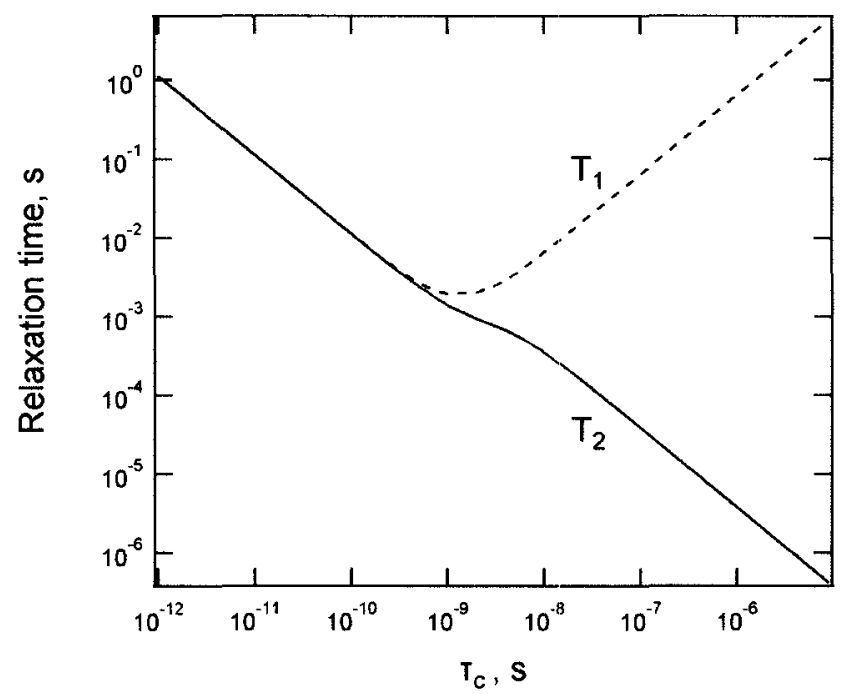

Figure C.1. Dependence of $T_{1}$ (dashed line) and $T_{2}$ (solid line) relaxation times on the correlation time $T_{c}$ for intramolecular quadrupolar relaxation (logarithmical axes are used). 
For ${ }^{2} \mathrm{H}, \eta \leq 0.2$ [142] and the asymmetry can be neglected to a good approximation. In the extreme narrowing limit, $J(0)=J\left(\omega_{0}\right)=J\left(2 \omega_{0}\right)=T_{c}$, and equations for the relaxation rates further reduce to

$$
\mathrm{R}_{1}=\mathrm{R}_{2}=\frac{1}{\mathrm{~T}_{1}}=\frac{1}{\mathrm{~T}_{2}}=5 \mathrm{C}_{\mathrm{c}}
$$

from which the correlation time can be calculated if either relaxation time and the quadrupolar coupling are known. For convenience, a plot and simple regression curve are shown in Figure C.2. We have used the constant $\mathrm{C}=1.78 \times 10^{11}\left(\mathrm{~Hz}^{2}\right)$, based on the known ${ }^{2} \mathrm{H}$ quadrupolar coupling constant at room temperature (liquid ${ }^{2} \mathrm{H}_{2} \mathrm{O}$ ), $\mathrm{v}_{\mathrm{q}}=245 \mathrm{kHz}[142]$.

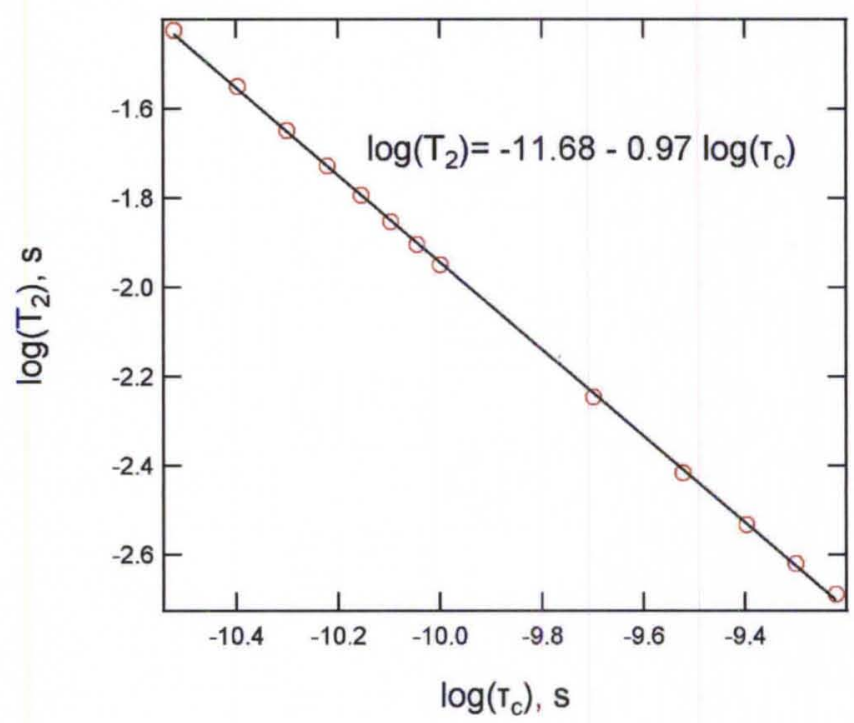

Figure C.2. Linear approximation (solid line) of the dependence of $T_{2}$ (red circles) on the correlation time $T_{c}$ in the range of relevant $T_{2}$ values. 


\section{APPENDIX D}

\section{LIST OF SYMBOLS AND ABBREVIATIONS}

$\varphi$ - basis function

$\rho$ - density operator

$\sigma$-chemical shielding tensor with principal components $\sigma_{11}, \sigma_{22}, \sigma_{33}$

$T_{c}$ - rotational correlation time

$\omega_{0}-$ Larmor precession frequency

$\omega_{q}$ - quadrupolar coupling frequency, $\omega_{q}=2 \pi v_{q}$

$\Omega$ - observable

$\Psi$ - wavefunction

1D and 2D spectra - one- and two-dimensional spectra

$1 \mathrm{Q}, 2 \mathrm{Q}, 3 \mathrm{Q}, 4 \mathrm{Q}$ and $5 \mathrm{Q}$ - single, double, triple, quadruple and pentuple quantum

$A_{2 Q}-2 Q$ signal intensity

$\mathrm{CDCl}_{3}$ - deuterated chloroform

$\mathrm{CL}$, TOCL - cardiolipin, tetraoleoyl cardiolipin

CSA - Chemical Shift Anisotropy

D - self-diffusion constant

DMSO - dimethyl sulfoxide 
$F_{1}, F_{2}$ and $F_{3}-$ direct, indirect and detection dimensions

FID - Free Induction Decay

$J\left(\omega_{0}\right)$ - spectral density

$\mathrm{H}$ - interaction Hamiltonian

$\mathrm{H}_{3} \mathrm{PO}_{4}$ - phosphoric acid

$\mathrm{H}_{\|}$- inverted hexagonal phase

HSQC - Heteronuclear Single-Quantum Correlation spectroscopy

I - spin quantum number

$\mathrm{L}_{a}$ - lamellar, liquid crystalline phase

$\mathrm{L}_{\beta}$ - gel phase

$L_{G}, L_{M H}$ and $L_{s}$ - ground, matching and sample coil inductances

MAS - Magic Angle Spinning

MD - Molecular Dynamics

MQ - Multiple Quantum

MRI - Magnetic Resonance Imaging

$\mathrm{NaHM}$ - sodium hydrogen maleate

PA - phosphatidic acid

PC - phosphatidylcholine

PEG - polyethylene glycol

$P^{e q}(\Omega)$ - normalized orientational distribution function

RF pulse - radiofrequency pulse

$\mathrm{pK}_{\mathrm{a}}-$ acid dissociation constant

$\mathbf{s}-$ span, $\mathbf{s}=\sigma_{33}-\sigma_{11}$ 
$S^{2}$ - generalized order parameter

$S^{(2)}-$ order parameter

$S_{B}-$ Boltzmann entropy

$\mathrm{S} / \mathrm{N}$ - signal to noise ratio

SSNMR - Solid-State Nuclear Magnetic Resonance spectroscopy

$\mathrm{T}_{1}$ - longitudinal relaxation time

$\mathrm{T}_{2}^{2 \mathrm{Q}}$ - transverse relaxation time of the $2 \mathrm{Q}$ signal

$T_{2}^{\text {pure }}$ - transverse relaxation time of the solvent alone, sample is "pure" of protein

$\mathrm{T}_{2}^{\text {bulk }}$ - transverse relaxation time of the bulk solvent, no $2 Q$ filtration

$\mathrm{T}_{2}^{\text {surf }}-$ transverse relaxation time of the solvent ordered at the protein surface,

$2 Q$ filtered signal

$\mathrm{Tr}$ - trace

$v_{\text {rot }}$ - spinning speed of the rotor

$\mathrm{w}_{\text {so }} / \mathrm{w}_{\text {pro }}$ - solvent/protein mass (weight) ratio 


\section{CURRICULUM VITAE}

NAME: $\quad$ Tatiana Victorovna Krivokhizhina

DOB: $\quad$ Saratov, Russia - September 18, 1981

ADDRESS: $\quad 2008$ Murray Ave \#1, Louisville, KY 40205

E-MAIL: $\quad$ krivokhizhina@gmail.com

$\begin{array}{lll}\text { EDUCATION: } & \text { Ph.D., Biochemistry } & 2012\end{array}$

University of Louisville, Louisville, KY

Dissertation: Applications of Solid State NMR to

Cardiolipin and Elastin

Ph.D., Physics

2006

Saratov State University, Saratov, Russia

Dissertation: Quantum-Mechanical Investigation of Vibrational

Specrta of Anthracene Derivatives and Corrole Complexes

M.S., Physics

2003

Saratov State University, Saratov, Russia

Diploma with Honors

Thesis: Calculation of Vibrational Spectrum of Antraquinone by

Method of Density Functional 


\section{RESEARCH EXPERIENCE:}

Solid-state Nuclear Magnetic Resonance Laboratory $2006-2012$ Research advisor: Dr. Richard J. Wittebort UofL, Louisville, KY

Development of advanced pulse sequences for the detection of multiple quantum coherences from weakly ordered water in elastin. The goal of this work is to understand the mechanism for elastic recoil in nature's most important elastomer.

Design and implementation of ${ }^{1} \mathrm{H},{ }^{13} \mathrm{C}$ and ${ }^{31} \mathrm{P}$ NMR experiments for the investigation of structural hypothesis that phospholipid cardiolipin functions as a proton trap in the membranes of energy producing organelles.

NMR hardware experience in building double and triple resonance probes for the homebuilt spectrometer.

\section{Computational Chemistry Laboratory}

Research advisor: Dr. Pawel M. Kozlowski
$2005-2006$ UofL, Louisville, KY

Interpretation and correlation of vibrations for complexes of corrole with transition metal ions: $\mathrm{Cr}, \mathrm{Mn}, \mathrm{Fe}, \mathrm{Co}, \mathrm{Ni}, \mathrm{Cu}, \mathrm{Zn}$ and $\mathrm{Ga}$. Determination of analogous vibrations in metalo-porphyrins.

Determination of the effect of metal ion substitution on the structure and vibrational frequencies of corrole.

Comparison of spatial structures and full energies of monodeprotonated and protonated $\mathrm{NH}$-tautomers of corrole obtained for the first time.

\section{Optics and Molecular Spectroscopy Laboratory}

Research advisors: Dr. Vladimir V. Nechaev
$2000-2005$

SSU, Saratov

Russia

Dr. Kirill V. Berezin

Performance of quantum-mechanical calculations to establish correlation of vibrationalmodes of anthracene and its derivatives: acridone, hydrogenbonded dimer of acridone, $\mathrm{N}$-deuterated acridone, 9,10-anthraquinone and four isotopomers of anthraquinone.

Assignment and interpretation of experimental IR and Raman spectra of anthracene and its derivatives using calculated vibrational frequencies. 
PRESENTATIONS:

Gordon Research Conference, Magnetic Resonance

Biddeford, ME

Poster presentation, "Mechanism of Elastin's Elasticity by MQ NMR"

2011 American Chemical Society 42nd Central Regional Meeting Indianapolis, IN Oral presentation, "Cardiolipin as a proton trap:

Investigation by solid state NMR"

International Conference for Undergraduate and Graduate Moscow, Russia Students in Fundamental Sciences - "Lomonosov-2004"

Oral presentation, "Quantum mechanical analysis

of vibrational states of 9,10-anthraquinone"

II Russian Conference on Molecular modeling in Chemistry, Saratov, Russia Biology and Medicine 2004

Oral presentation, "Quantum mechanical analysis of IR and Raman spectra of acridone taking into account intermolecular $\mathrm{H}$-bond formation" International School for Junior Scientists and Students Saratov, Russia on Optics, Laser Physics and Biophysics 2002

Oral presentation, "Application of frequency scaling method

in quantum mechanical calculations of vibrational molecular spectra"

\section{PUBLICATIONS:}

Berezin K.V., Krivokhizhina T.V., and Nechaev V.V. Theoretical analysis of the IR and Raman spectra of acridone taking into account intermolecular hydrogen bonds. Optics and Spectroscopy, 2006. 100(1): p.15-22. 
Berezin K.V., Krivokhizhina T.V., and Nechaev V.V. Atypical intensity distribution in the Raman spectrum of 9,10-anthraquinone. Optics and Spectroscopy, 2004. 97(4): p. 530-536.

Berezin K.V., Nechaev V.V., and Krivokhizhina T.V. Application of a method of linear scaling of frequencies in calculations of the normal vibrations of polyatomic molecules. Optics and Spectroscopy, 2003. 94(3): p.357-360.

TEACHING EXPERIENCE:

University of Louisville Louisville, KY

Graduate Teaching Assistant $2005-2012$

Physical Chemistry Laboratory (470)

Organic Chemistry I Laboratory (343)

Introduction to Chemical Analysis I and II Laboratory (207/208)

General Chemistry I and II Recitation (201/202)

Introductory Chemistry Laboratory (103)

Saratov State University

Saratov, Russia

Graduate Teaching Assistant $2003-2005$

Introduction to Atomic Physics Laboratory

Introduction to Optics Laboratory

Introduction to Molecular Physics Laboratory

PROFESSIONAL SOCIETIES: American Chemical Society 